\title{
Effects of Imbalance and Geometric Error on Precision Grinding Machines
}

\author{
J. E. Bibler
}

(MS Thesis)

June 1, 1997

This is a preprint of a paper intended for publication in a journal or proceedinga. Since changes may be made before publication, this preprint is made available with the underatanding that it will not be cited or reproduced without the permieaion of the author. 


\section{DISCLAIMER}

This document was prepared as an account of work sponsored by an agency of the United States Government. Neither the United States Government nor the University of California nor any of their employees, makes any warranty, express or implied, or assumes any legal liability or responsibility for the accuracy, completeness, or usefulness of any information, apparatus, product, or process disclosed, or represents that its use would not infringe privately owned rights. Reference herein to any specific commercial product, process, or service by trade name, trademark, manufacturer, or otherwise, does not necessarily constitute or imply its endorsement, recommendation, or favoring by the United States Government or the University of California. The views and opinions of authors expressed herein do not necessarily state or reflect those of the United States Government or the University of California, and shall not be used for advertising or product endorsement purposes.

This report has been reproduced directly from the best available copy.

Available to DOE and DOE contractors from the Office of Scientific and Technical Information P.O. Box 62, Oak Ridge, TN 37831

Prices available from (615) 576-8401, FTS 626-8401

Available to the public from the National Technical Information Service

U.S. Department of Commerce 5285 Port Royal Rd., Springfield, VA 22161

Work performed under the auspices of the U.S. Department of Energy by Lawrence Livermore National Laboratory under Contract W-7405-ENG-48. 


\title{
Effects of Imbalance and Geometric Error on Precision Grinding Machines
}

\author{
J. E. Bibler
}

Manuscript date: June 1, 1997

LAWRENCE LIVERMORE NATIONAL LABORATORY University of California • Livermore, California • 94551 



\title{
Effects of Imbalance and Geometric Error on Precision Grinding Machines
}

\author{
by \\ Jared Evan Bibler \\ Bachelor of Science in Mechanical Engineering \\ Massachusetts Institute of Technology \\ June 1996
}

Submitted to the Department of Mechanical Engineering in partial fulfillment of the requirements for the degree of

Master of Science in Mechanical Engineering

at the

\section{MASSACHUSETTS INSTITUTE OF TECHNOLOGY}

June 1997

\section{(C)1997 Jared Bibler}

All rights reserved

The author hereby grants to MIT permission to reproduce and to distribute publicly paper and electronic copies of this thesis document in whole or in part.

Signature of Author

Department of Mechanical Engineering May 20, 1997

Certified by

David L. Trumper

Rockwell International Associate Professor of Mechanical Engineering

Certified by

Kenneth L. Blaedel

Group Leader, Lawrence Livermore National Laboratory

Accepted by

Ain A. Sonin

Chairman, Departmental Graduate Thesis Committee 



\title{
Effects of Imbalance and Geometric Error on Precision Grinding Machines \\ by
}

\author{
Jared Evan Bibler
}

\author{
Submitted to the Department of Mechanical Engineering \\ on May 20, 1997, in partial fulfillment of the \\ requirements for the degree of \\ Master of Science in Mechanical Engineering
}

\begin{abstract}
This thesis examines effects of imbalance and geometric decenter on a precision grinding machine. Two models and the results of experimentation on an air spindle-load cell system are presented. A lumped-parameter model for a large grinding machine is presented, along with two models for the experimental system. Experimental data for the system includes modal analysis, spindle error motion, and force data over a range of operating speeds. Effects of adding damping, increasing stiffness, and compensating for geomteric decenter with imbalance are covered. Recommendations for extension of tests and application to industrial machining centers are also covered.
\end{abstract}

Thesis Supervisor: David L. Trumper

Title: Rockwell International Associate Professor of Mechanical Engineering

Thesis Supervisor: Kenneth L. Blaedel

Title: Group Leader, Lawrence Livermore National Laboratory 


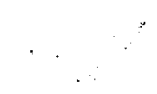

. 
Dedicated to the memory of Walter Bibler (1911 - 1996). 



\section{Acknowledgements}

I would like to thank Ken Blaedel for being an excellent mentor, and for putting up with my hourly interruptions, as I put up with his Cheesehead ways. I could not have gathered a single bit of data if it were not for the continual help and expertise of Nhan Nguyen. The modal tests would have been impossible without Tom Woehrle. I would also like to thank Mark Piscotty (thanks for nothin' Fast MP!) and Debbie Krulewich for being great neighbors and answering countless questions. Thanks also to Charlie Cass for bringing metal to life with his machining. Professor David Trumper was a great inspiration to me during some tough times in the research. Finally, Bob Langland and Katie Broughton deserve accolades for their help and proactive support over the years. 



\section{Contents}

1 Background and Introduction $\quad 16$

1.1 General Introduction to Grinding . . . . . . . . . . . . . . . . 16

1.2 Defining the Study of Balance Errors . . . . . . . . . . . . 16

1.3 Application of the Study . . . . . . . . . . . . . . 17

1.4 Is balancing worthy of study? . . . . . . . . . . . . 18

1.5 Overview of this Study . . . . . . . . . . . . . . . . 19

1.6 Literature Review . . . . . . . . . . . . . . . . 19

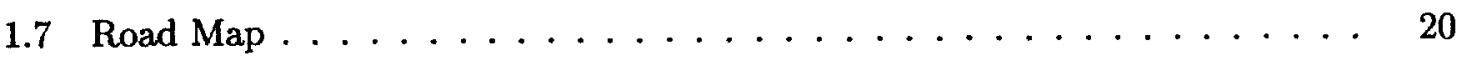

2 Project Description 22

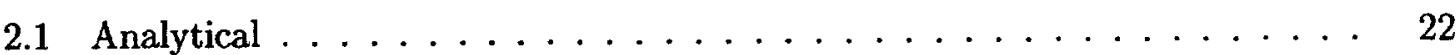

2.2 Experimental . . . . . . . . . . . . . . . . . . 24

2.3 Comparing Analysis and Experiment . . . . . . . . . . . 26

3 Experimental Apparatus $\quad 27$

3.1 Mechanical System . . . . . . . . . . . . . . 27

3.2 Data Acquisition . . . . . . . . . . . . . . 33

4 Empirical Modal Analysis $\quad 37$

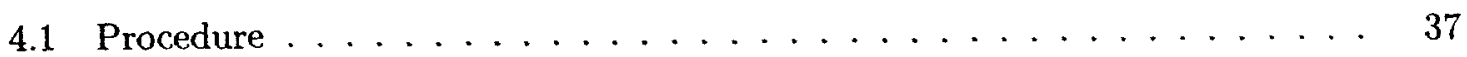

4.2 Results . . . . . . . . . . . . . . . . . 40

4.2 .1 Westwind-JR3 system . . . . . . . . . . . . . 41

4.2 .2 Westwind-Kistler system . . . . . . . . . . . . . 44

4.2 .3 Westwind $($ no load cell) $\ldots \ldots \ldots \ldots 45$

5 Models $\quad 48$ 
5.1 General Model . . . . . . . . . . . . . . . . . . 48

5.1 .1 Description .......................... 48

5.1 .2 Simulation Results . . . . . . . . . . . . . . . 51

5.2 Explicit Analytical Model . . . . . . . . . . . . . . 55

5.3 Implicit STAR Model $\ldots \ldots \ldots \ldots \ldots \ldots \ldots$

6 Experimental and Model Results $\quad 61$

6.1 Sample Experimental and Model Outputs . . . . . . . . . . . . 61

6.2 Undamped JR3 System: Empirical Error Motion and Two Models . . . . 64

6.3 Kistler System: Empirical Error Motion and STAR Predictions . . . . . 72

6.4 Discussion of Error Motion Results . . . . . . . . . . . . . 76

6.5 JR3 System: Effects of Adding Damping Material $\ldots \ldots \ldots \ldots$

6.6 Force Transmitted to Machine Structure . . . . . . . . . . . . . . . 82

6.7 Compensating for Decenter with Imbalance . . . . . . . . . . . . . 84

6.8 Effect of Increasing Mass of Imbalance . . . . . . . . . . . . . 86

7 Experimental Challenges $\quad 89$

7.1 "Noise" and Natural Modes . . . . . . . . . . . . . . . . . . 89

7.2 Clock Speed Differences $\ldots \ldots \ldots \ldots$. . . . . . . . . . . 94

7.3 Metrology Ball Drift . . . . . . . . . . . . . . . . . . 94

7.4 Spindle Analyzer and Anti-Aliasing Filter . . . . . . . . . . 95

8 Recommendations $\quad 97$

8.1 Summary of Results . . . . . . . . . . . . . . . . . 97

8.2 Extension of Study . . . . . . . . . . . . . . . . . 99

8.3 Industrial Applications . . . . . . . . . . . . . . . . . . . . 101

A MATLAB Script for General Grinding Model $\quad 102$

B MATLAB Script for Analytical Grinding Model $\quad 105$

B.1 ODE solver . . . . . . . . . . . . . . . . . . . . . 105

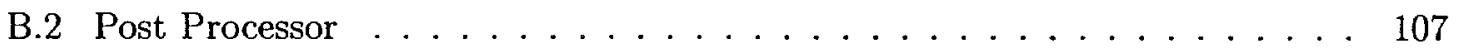

$\begin{array}{ll}C \text { Data Reduction } & 109\end{array}$

C.1 MATLAB Procedure . . . . . . . . . . . . . . . . . 109 


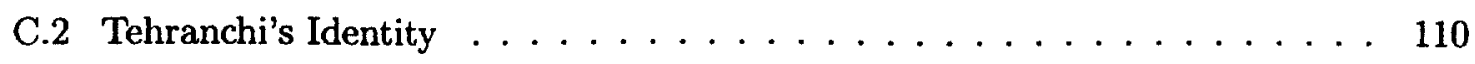

D Comparing Westwind Stiffness with Load Cell Stiffness 


\section{List of Figures}

3-1 Schematic of mechanical system: spindle, load cell, and capacitance gauges. 28

3-2 Cross-section of metrology ball, showing flexure shaft. . . . . . . . . 31

3-3 Top view schematic of spindle analyzer setup showing $1 /$ rev counter. . . . . 32

3-4 Flowchart for 12-channel data acquisition with JR3 dynamometer. . . . . 34

3-5 Synchronization scheme for 12-channel data acquisition using two CPUs. . . 35

3-6 Flowchart for 8-channel data acquisition with Kistler dynamometer. . . . . 36

4-1 Mesh used in STAR JR3 tests. . . . . . . . . . . . . . . . 39

4-2 Undamped JR3 system, mode 1, 96.7 Hz. Rotation about $\mathrm{Y}$ axis of load cell. 42

4-3 Undamped JR3 system, mode 2, 113.3 Hz. Rotation about X axis of load cell. 43

4-4 Undamped JR3 system, mode 4, $163.3 \mathrm{~Hz}$. Rotation about $\mathrm{Z}$ axis of load cell. 43

4-5 Undamped JR3 system, mode $5,289.5 \mathrm{~Hz}$. Combined translation and rotation. 44

4-6 System with no load cell, rotor moving axially, $456.5 \mathrm{~Hz} \ldots \ldots \ldots$. . . . 47

$5-1 \quad$ Dynamic grinding model. . . . . . . . . . . . . . . . 49

5-2 Effect of varying spindle stiffness on grinding process force and force transmitted to spindle. . . . . . . . . . . . . . . . 51

5-3 Effect of increasing process damping on spindle excursion. . . . . . . . 53

5-4 Magnitude transfer function between imbalance driving force and process force for ASPE model. . . . . . . . . . . . . . . . . . . 54

6-1 Sample data trace from experiment, $3000 \mathrm{rpm}$ with undamped JR3 system. 62

6-2 Sample output trace from analytical model, $9000 \mathrm{rpm}$ with undamped JR3 system. Displacements are in microinches. . . . . . . . . . . . 63

6-3 JR3 system without damping material, as sensed in the $\mathrm{X}$ (axial) direction; comparison of data and 2 models for displacement amplitude. . . . . . . . 65 
6-4 JR3 system without damping material, as sensed in the $\mathrm{X}$ (axial) direction; comparison of data and 2 models for phase relative to excitation input. . . 68

6-5 JR3 system without damping material, as sensed in the $Y$ (radial1) direction; comparison of data and 2 models for displacement amplitude. . . . . . . . .

6-6 JR3 system without damping material, as sensed in the $\mathrm{Y}$ (radial1) direction; comparison of data and 2 models for phase relative to excitation input. . . .

6-7 JR3 system without damping material, as sensed in the Z (-radial2) direction; comparison of data and 2 models for displacement amplitude. . . . . . . .

6-8 JR3 system without damping material, as sensed in the Z (-radial2) direction; comparison of data and 2 models for phase relative to excitation input. . . .

6-9 Kistler system, as sensed in the $\mathrm{X}$ (axial) direction; comparison of data and STAR model. . . . . . . . . . . . . . . . . . .

6-10 Kistler system, as sensed in the $\mathrm{Y}$ (radial1) direction; comparison of data and STAR model. . . . . . . . . . . . . . .

6-11 Kistler system, as sensed in the $\mathrm{Z}$ (-radial2) direction; comparison of data and STAR model. . . . . . . . . . . . . . . .

6-12 JR3 system with and without damping material, as sensed in the $X$ (axial) direction; capacitance-gauge and STAR simulation results for displacement amplitude. . . . . . . . . . . . . . . .

6-13 JR3 system with and without damping material, as sensed in the Y (radial1) direction; capacitance-gauge and STAR simulation results for displacement amplitude. . . . . . . . . . . . . . . .

6-14 JR3 system with and without damping material, as sensed in the Z (-radial2) direction; capacitance-gauge and STAR simulation results for displacement amplitude. . . . . . . . . . . . . . . . . .

6-15 Comparison of force into machine structure, Y direction, JR3 and Kistler systems. . . . . . . . . . . . . . . . .

6-16 Compensating for decenter with imbalance: the effect of phase lead on normalized decenter. . . . . . . . . . . . . . . . . .

6-17 Effect of increasing mass of imbalance on error motion in the Y-direction, at

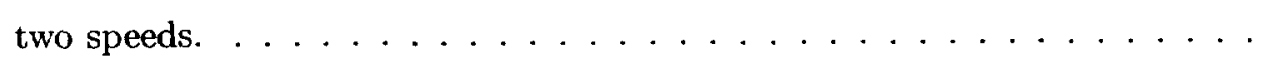


6-18 Effect of increasing mass of imbalance on force in the Y-direction, at two

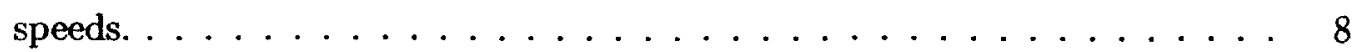

7-1 Sample graph of frequency domain output for load cell; spindle excited at nose and sensed in each of three directions. (Directions are as defined in

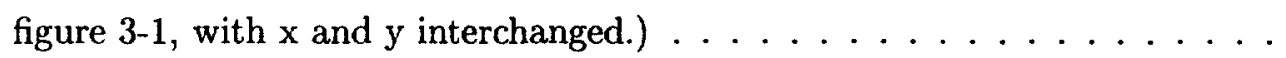

C-1 Effect of correcting the capacitance gauge output for initial geometric decenter: trace of radiall (Y) cap gauge on Kistler system. . . . . . . . . . . . 111 


\section{List of Tables}

3.1 Stiffness of JR3 Load Cell . . . . . . . . . . . . . . . . . . . . . 29

3.2 Stiffness of Kistler Load Cell _ . . . . . . . . . . . . . . . 30

3.3 Imbalance Masses . . . . . . . . . . . . . . . . . . . . . . 30

4.1 JR3, no damping: natural frequencies and mode shape descriptions. *-see

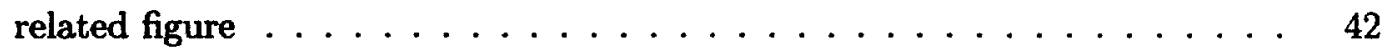

4.2 JR3 with damping: natural frequencies and mode shape descriptions . . . 44

4.3 Kistler: natural frequencies and mode shape descriptions . . . . . . . . . 45

4.4 Spindle natural frequencies and mode shape descriptions (no load cell) *-see related figure ........................... 46

5.1 Modes of JR3-Westwind system used in analytical model . . . . . . . . . 55

5.2 Moments about load cell axes . . . . . . . . . . . . . . . 56

5.3 Moments and equivalent stiffnesses for six modes . . . . . . . . . . 57 


\section{Chapter 1}

\section{Background and Introduction}

\subsection{General Introduction to Grinding}

Grinding is an important process in many areas of manufacturing. Historically, grinding has been used as both a coarse process, for high-speed material removal, and as a fine process for finishing applications. There is intense interest in the manufacturing industry in improving grinding, to yield improvements in figure precision, surface finish, and cost. There is also interest in broadening the uses of grinding, to include ductile-mode material removal for brittle materials, such as optical glass. At Lawrence Livermore National Laboratory one use of precision grinding under study is the manufacture of precision optics. The quest for high-precision parts in optics and other fields is driving a new look at the fundamentals of grinding.

\subsection{Defining the Study of Balance Errors}

At issue in this thesis is the effect of balance on grinding. Balance error is caused by a mass or mass concentration that is not symmetrically distributed about the axis of rotation. This imbalance results in a time-varying force normal to the axis of rotation. In any one sensitive direction, the imbalance looks like a sinusoidal force oscillation; it is also, and more completely, a force vector which rotates around with the machinery, having a period of once per revolution. The magnitude of the imbalance force changes as the square of the rotational speed. An imbalance can be thought of as a lump of dough stuck onto a rolling pin. When the rolling pin is spun, the dough causes a wobble in the spinning motion. 
The nonuniform mass of the dough stuck to the rolling pin results in a time-varying force normal to the axis of rotation. This is caused by non-uniform centripetal acceleration in the spinning shaft. The equation for force magnitude of an imbalance is

$$
F=m r \omega^{2}
$$

where $m$ is the mass of imbalance, $r$ is the distance out from the axis of rotation, and $\omega$ is

the rotational speed. This equation can be easily derived from first principles of rotating objects; it is the magnitude of the sine term which describes unidirectional force oscillation due to imbalance. Alternately, it may be thought of as the unidirectional component of the constant magnitude imbalance phasor, which rotates with the spindle. In grinding, the same phenomenon as the rolling pin occurs when there is a nonsymmetric "lump" of mass in the rotational part of the grinding system. This "lump" of mass may be the product of an extremely subtle geometric nonuniformity: nonuniform mass distribution around the grinding wheel, nonsymmetric fixturing, or uneven mass distribution in the grinding spindle itself, for example.

In a precision machining system, it is often useful to determine an error budget which compares the results of different sources of error on the total dimensional error of the machined part. The imbalance force can be looked at as one element of the total error budget for the grinding process. There are many other (some undetermined) sources of error in grinding. Examples include thermal errors, seismic errors, and out-of-roundness errors. Added precision in grinding can be enabled through a detailed study of the dimensional error budget. The overall balance error is a combination of balance errors in the spindle, fixturing, and grinding wheel. Imbalance is an error source which directly couples with machine dynamics: it can excite natural modes of the machine tool, which can in turn worsen the spindle error motion and ultimately contribute to part errors.

\subsection{Application of the Study}

A thorough study of the effects of imbalance on precision grinding will give Lawrence Livermore the ability to produce better-quality parts at lower cost. Ultimately, engineers and machinists will be able to specify a balancing tolerance for the machine based on a given part tolerance. They will be able to pick a grinding speed based on machine dynamics, 
to minimize errors on the part. They may even use known imbalances to compensate for geometric errors in the grinding wheel. This will produce parts of better form (including surface waviness) and finish. Engineers and machinists would also have the ability to weigh the relative importance of balance errors against other error sources. This could reduce cost as personnel will be able to make an informed decision regarding the necessity of balancing the system and to what degree it needs to be balanced. This thesis will be a first step in the direction of a more thorough understanding of balance.

\subsection{Is balancing worthy of study?}

Balancing in grinding is a problem worthy of study. One reason is that balancing errors may not be fully correctable, so it is important to determine to what extent they affect the quality of the work. Typically, a balance error is removed by first "truing" the grinding wheel and then adding mass to specific locations on the wheel to minimize the force magnitude given in equation 1.1. The act of truing is cutting the wheel at speed so that it is of uniform roundness in a radial sense and planar in an axial sense. A wheel which has not been trued will have "high spots" or "low spots" so that the wheel's contact with the workpiece is not uniform throughout the rotation of the wheel. But truing is not completely effective. It is a single-point correction of the wheel's geometry and in some types of grinding (for example, spherical) there is a large contact line between the wheel and the workpiece. Also a grinding wheel can never be perfectly trued, to zero runout, since at some level the force required to true the wheel will be greater than the force needed to bend the wheel away from the truing tool. Another reason balancing errors are not fully correctable is that imbalance may be generated during the grinding process itself: if the wheel wear is nonuniform, for example. Finally, the study of balancing in grinding is a challenging problem. It requires a familiarity with the concepts and terminology of precision engineering. It requires a thorough experimental effort including high-speed data acquisition, high-speed spindle analysis, and modal analysis of the grinding system. Careful modeling of the grinding process can also yield valuable insight, although this is still an emerging area in the literature. 


\subsection{Overview of this Study}

To study balancing in grinding, I examined a simple mechanical system. It was essential to study such a well-defined system, as opposed to a large, complex system such as a machining center. The use of a compact, well-defined system enabled easy quantification of the imbalance force input, its phase angle to any geometric decentering, and good understanding of the machine mode shapes. It is important to understand a simple system such as the one I examined given that imbalance is so intimately coupled to machine dynamics. It is possible to extend the results presented here to industrial machines, although that is not part of this work.

In addition to the empirical testing, I modeled the simple mechanical system to look at how mode shapes, balance, and geometric error interplay to yield spindle error motion. I will present the results of this model along with the results from a more global grinding model. The global model, presented at ASPE in November 1996 [1] allows one to examine the effects of changing global machine parameters like stiffness and damping. This geometrically abstract, one-dimensional model will be presented to demonstrate the usefulness of an abstract approach for first-order understanding but it will not be the main focus of this thesis.

\subsection{Literature Review}

In the early stages of this project, I did an extensive literature search and found some literature relevant to the study of effects of balance in grinding. The first area of interest is the related field of chatter in machine tools. There are many articles on chatter in the literature and although chatter is a different phenomenon than imbalance, the literature can be instructive in that it links material removal processes with machine dynamics. This literature is useful as background in the derivation of a dynamic grinding model. One article which was of particular interest to this project, since it suggested a cutting process modelled as a damper, is [13].

There are a wide variety of grinding process models available in the literature, but many times these are not extendable past the specific application for which they were derived. A very simple and useful grinding process model for this project was found in [8]. Additionally useful was [11]. This model was chosen for its simplicity and applicability to our problem 
through experiment. However, an excellent survey paper exists which covers about fifty different process models for grinding [19]. The models surveyed in this paper cover many specifics of grinding which are beyond the scope of this thesis, such as material surface integrity and thermal effects. The paper is an excellent starting-point for any advanced attempts at modelling the grinding process.

There are several useful references on balancing of high-speed machinery. A good start in this area is [3], which gives the reader a good background in various global approaches to machine balancing. In addition, there are many textbooks on rotor dynamics. Two good references in this field can be found in [4] and [15]. There is some literature pertaining

specifically to balancing of grinding wheels. Gawlak briefly describes a series of experiments done with unbalanced wheels to determine workpiece waviness [6], while Layne discusses approaches for controlling imbalance in-process [12]. Generally helpful to me throughout the course of this project was the staff at Balance Dynamics Corporation, a manufacturer of in-process system balancing equipment. One of their internal papers provides a good look at the effect of using their proprietary balancer on grinding wheel excursion [2].

One of the most useful publications in this work was the ANSI Axis of Rotation Standard [7]. The standard was helpful in designing experiments to measure spindle error and characterizing spindle error. It is highly recommended by the author for any work involving precision spindles. Also of interest is the ISO balance standard for rigid rotors [5]. This gives curves for allowable imbalances in machining, broken down by process type.

\subsection{Road Map}

This thesis is organized as follows. In Chapter Two, I describe the experimental and analytical sequence and the motivation behind it. Chapter Three describes the experimental hardware used in the project. Chapter Four discusses the modal analysis performed as part of the project. Chapter Five deals with models of the grinding systems: the first section discusses a general one-dimensional model, while the remaining two sections discuss an analytical model and a modal analysis software model to predict spindle error motions due to imbalance in the grinding system tested in this thesis. Chapter Six presents the results of experiments performed on the grinding system alongside the results for the two models. Chapter Seven talks about some of the problems I faced in carrying out the project, so that 
others might learn from my experiences. Chapter Eight recommends extensions of the tests and discusses industrial applications of the results. 


\section{Chapter 2}

\section{Project Description}

\subsection{Analytical}

The goal of the analytical portion of the project is to understand imbalance as a driver of machine dynamics. Unlike some of the other potential error sources in a machining operation, imbalance directly excites resonances in the machine tool itself. One could liken an unbalanced rotor to a shaker, used in dynamic testing. In a given plane containing the axis of rotation, the imbalance looks like a sinusoidal force input, occurring at the shaft rotational frequency. For this reason, imbalance could potentially cause catastrophic errors in the part, as well as damage to the machine itself, in an extreme case. More commonly, imbalance will deteriorate form accuracy or surface finish. Understanding of machine oscillations generated by an imbalance is important because it is machine oscillations which lead directly to spindle error motion (an observable error in the instantaneous location of the spindle axis of rotation). Spindle error motion is then transmitted through the cutting process into errors on the part.

It should be noted here that rotors can exhibit nonsynchronous (i.e. occurring at a frequency other than once per revolution) effects such as whip and whirl [4, pages 1.731.78]. Whirl always occurs at the natural frequency of the shaft, regardless of the frequency of rotation. (For the spindle used here the natural frequency of the shaft is on the order of $1000 \mathrm{~Hz}$.) Whirl and whip, motions of the rotor out of the plane containing the axis of rotation, are usually brought on by nonlinear effects within the rotor (many of them hydrodynamic or friction-induced). These phenomena, while potentially important secondorder effects, will not be addressed in this thesis. 
There are two distinct purposes for the models that will be presented here. The first purpose is to understand how imbalance may affect the dynamics of a large machine tool, in one dimension. A global model allows parameters within the grinder, such as stiffness, damping, and the grinding process model to be varied. The second purpose is to describe the dynamics of a "simple" grinding system in three dimensions.

From each distinct purpose comes a distinct model. The first model looks at how imbalance affects a generic machine tool. The results of this one-dimensional grinding model were presented at ASPE [1]. The model is geometrically abstract, including lumped parameters for stiffness, damping, and inertial terms. It is included in this thesis mainly as a demonstration of the usefulness of the approach for rudimentary understanding of a complex system like a grinding machine. This model has three distinct components: a rotary system (source of imbalance forces), a machine structure, and a material removal process. The most important contribution of this model is in expressing the potential complexity of machine dynamics. By numerically varying parameters in the model, for example air-film stiffness, one can get an understanding of how sensitive the parameter is to imbalance or any other disturbance. Since the model is abstract, it could be used to compare different types and sizes of grinding machine. This model is also useful because it is possible to apply to many other types of machine tools.

The second model is specific to the experimental air-bearing grinding system described in this thesis. As such, the geometric parameters are carefully chosen to reflect the real system as closely as possible. This model predicts spindle error motion in three axes for the experimental system from input imbalance force. The dynamics of the model are based on oscillatory modes observed in the system from modal analysis. The input frequency can be swept through the speed range of the system and error motion in each axis predicted at each speed. This model could be incorporated into a more global model by adding surrounding machine structures, a part spindle, and a cutting process model (although this will not be covered here). This model is derived from empirical knowledge about the oscillatory mode shapes and natural frequencies of the system.

In addition, there is a third model presented, which uses built-in features of the modal analysis package to predict displacements given an input sinusoidal force. This is much easier to implement than the analytical model; it is ready to run in the software at the conclusion of modal analysis. 


\subsection{Experimental}

The experimental portion of the project consists of characterizing a "simple" grinding system, a Westwind air bearing spindle (commercially available precision grinding spindle) atop either of a pair of dynamometers. The whole assembly is mounted to a large granite block, which is isolated from the floor by compressed-air pistons. In the operating frequency range, the granite block looks like mechanical ground to the system. Two dynamometers were chosen, a JR3 6-axis strain gauge load cell and a Kistler 3-axis (translation only) piezoelectric load cell. Being a piezoelectric cell, the Kistler is significantly stiffer than the JR3. Using the dynamometers serves two purposes: 1) they give a force output, analogous to the force felt by the machine structure in a more complete machine tool; 2) they add system dynamics by interacting with the very stiff grinding spindle. It is the second purpose that is the most interesting to this project: by adding a dynamometer we are essentially simulating a more complex structure in a compact and well-instrumented fashion. In addition to the two load cell systems, some data were taken with the air spindle bolted directly to the granite table. This much stiffer system showed very little in the way of resonant effects, and serves as a baseline to the other tests.

Three major types of data were taken for the Westwind/load-cell system. First, natural mode data were taken. Understanding the relevant natural modes of the system is crucial to understanding the effects of any dynamic excitation, such as imbalance. To accomplish this, a commercially-available modal analysis package, SMS Star, was used in conjunction with 3-axis accelerometers placed in 33 locations in a mesh around the system. The results of these tests show natural frequency and mode shape data for each natural mode of each of three systems (Westwind with JR3, Kistler, and no load cell).

Second, spindle error motion data were taken. Understanding spindle error motion is fundamental to a precision grinding system, as it is spindle error motion, carried through the cutting process, which maps onto the workpiece as surface figure or finish errors. To observe spindle error, an orthogonal set of three Pioneer capacitance gauges was used. The gauges measured the motion of a steel metrology ball which rotates with the spindle. Two gauges measure radial errors, one in the $y$-direction and one in the $z$-direction (see figure $3-1$ ). A third gauge measures axial error motion ( $x$-direction). The gauge outputs were amplified with Pioneer boards, passed through anti-aliasing filters, and then sampled at $25 \mathrm{kHz}$ with 


\section{a Pentium computer.}

Third, the outputs from the dynamometer were measured. These are important because as part of a larger machine the force transmitted to the table might excite machine modes. These were amplified with either a Kistler charge amplifier or a JR3 strain gauge amplifier, passed through an anti-aliasing filter, and sampled at $25 \mathrm{kHz}$ on a Tektronix 8-channel data acquisition system. In addition, a timing window which triggered a light sensor once per revolution was used as a reference pulse and sampled by the Pentium, as well as the Tektronix A/D boards.

Experiments on the system were designed according to questions that a machine or process designer might ask:

- "How fast should I cut?"

- "What difference does a 'softer' or 'stiffer' machine make?"

- "How are wheel truing and balance related?"

- "How does error motion scale with imbalance magnitude?"

- "If I add damping, will that improve the spindle error motion due to imbalance?"

To answer the first question, "How fast should I cut?", the effect of varying the spindle speed over a frequency range was tested, both at the best attainable level of balance, and with an imbalance weight attached to the spindle. The answer to the second question, "What difference does a 'softer' machine make?" came from changing the load cell under the spindle. This is analogous to changing the entire machine structure. This enables determination of which effects are load-cell independent and hence intrinsic to the spindle or instrumentation. Both "best" balance and the lowest level of imbalance were tested.

"How are wheel truing and balance related?" To answer this, a series of tests were run with known ball decenter and imbalance which varied in phase angle with respect to the decenter. The ball decenter was used in place of an untrue grinding wheel. These tests were run at several speeds, both with and without a load cell. In the case with the load cell, the tests were run both below and above all fundamental modes of the system.

To answer "How does error motion scale with imbalance magnitude?", a series of increasing masses were used and the spindle was spun at a variety of speeds, both at a system 
harmonic and below the system harmonics. Finally, the damping question was answered by adding a thin layer of Sorbothane damping material in parallel with the soft (JR3) load cell and measuring spindle error motion and dynamometer force both with and without a low level of imbalance. It is important to note that dynamometer force output between the undamped and damped JR3 systems is not directly comparable: the Sorbothane transmits force which is not measured by the load cell.

\subsection{Comparing Analysis and Experiment}

To tie the experimental data together with the modeling data, Bode-style frequency response plots were drawn that allow comparison of predictions and experimental results. These plots allow us to determine how relevant are the models at predicting important effects in the spindle. 


\section{Chapter 3}

\section{Experimental Apparatus}

\subsection{Mechanical System}

This chapter describes the setup I used to acquire empirical data on imbalance. The heart of the setup is a Westwind air bearing spindle, model D1150-03A. This is a commerciallyavailable precision grinding spindle with a speed rating of up to $50,000 \mathrm{rpm}$. The spindle requires a constant inflow of $80 \mathrm{psi}$ air to keep the rotor supported by the air bearings. The air, after passing through an air dryer, flows in through the back of the spindle and out the front around the rotor and through an exhaust hole. Inside the spindle, the air flows through passages which are oriented so as to provide stiffness in both the axial (thrust) and radial directions. It is important that the air be left on at all times. (With the air off, damage can easily occur if an air bearing is rotated even slightly.) There is no contact between the rotor and any part of the stator; the spindle motor turns the shaft through magnetic forces. The motor is a DC brushless device with a pulse width modulated power amplifier, and is controlled by a Volkmann motor drive controller. The spindle itself is a cylindrical unit; in this setup it was provided with an optional bottom-mount housing which the cylinder is attached to at the front side and cantilevered out the back. (This housing arrangement provides for some interesting vibrational modes, which will be covered later.) The nose of the spindle contains a taper chuck used to hold cutting tools, or in the case of these experiments, a metrology ball. Figure 3-1 shows a schematic of the Westwind spindle and other relevant apparatus.

Cooling water flows through the spindle to keep the temperature constant. We used a thermograph to keep track of the spindle and ambient temperatures while operating. The 


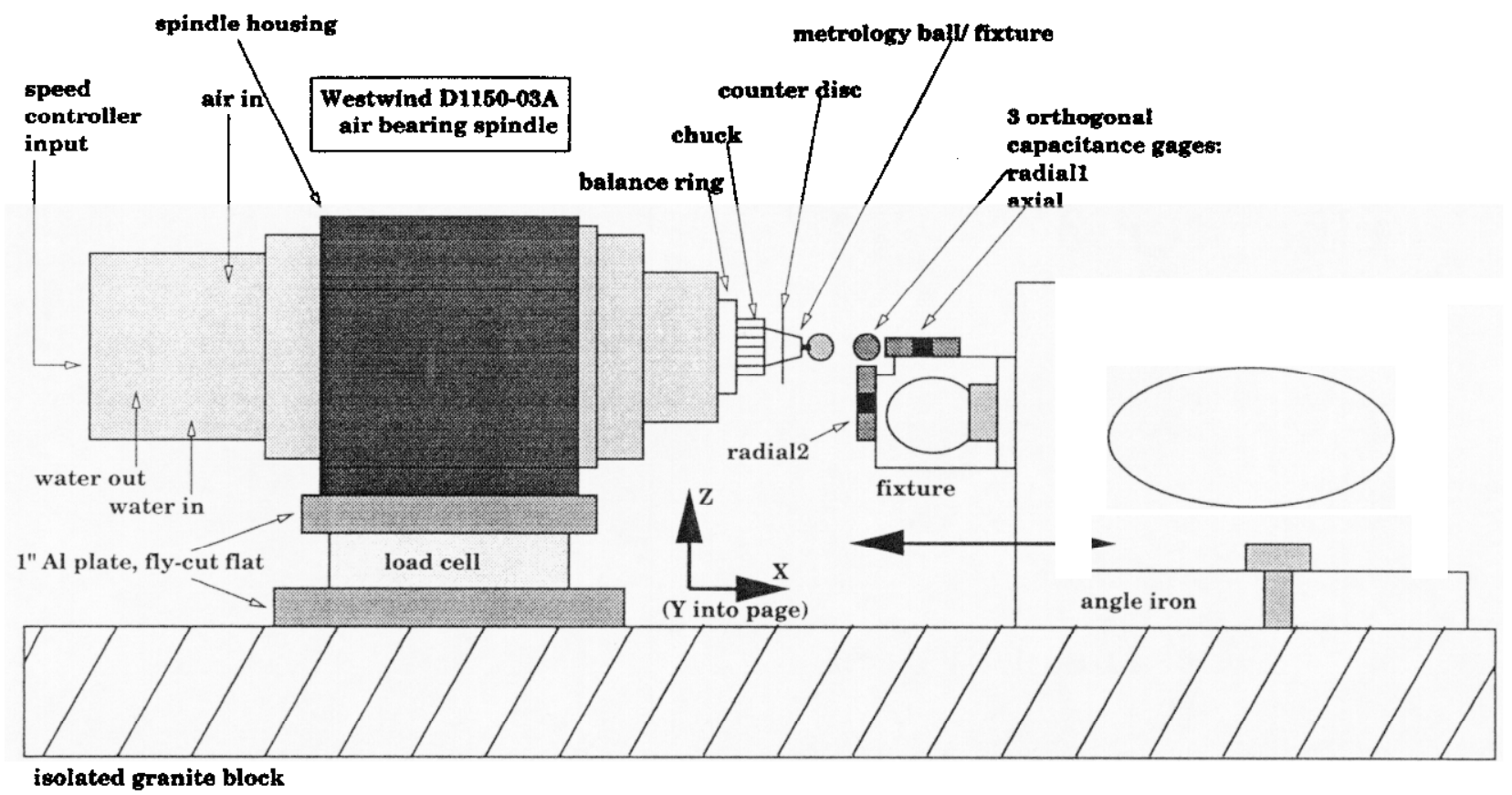

Figure 3-1: Schematic of mechanical system: spindle, load cell, and capacitance gauges.

water, supplied by the Laboratory's cooling water system, ensured that the temperature of the spindle stayed at $70 \mathrm{~F}$, fluctuating only $\pm 1.5 \mathrm{~F}$ over the course of a 24-hour period. Over the short time period of the experiments (on the order of a minute), temperature fluctuation was not a significant factor.

The spindle sits on a very large (16' long X 3' high X 3.5' deep) granite beam which was isolated from the floor on 4 corners. This large mass is chosen as a good approximation to mechanical "ground": its dynamics occur at frequencies lower than the ones of concern in the spindle tests. For example, the first bending mode of the block on its isolators is estimated to be at $30 \mathrm{~Hz}$. To attach the spindle to the granite block, we use three methods which, from a vibrations standpoint, comprise three completely different mechanical systems.

The most basic method of attachment involves clamping the spindle directly to the block using toe clamps. Of the three setups, this is by far the stiffest. The latter two methods are more involved, requiring load cells to be placed between the spindle and the table. I chose two very different load cells to examine. Both are mounted between 2 aluminum plates which have been fly-cut flat on both sides. The bottom plate is bolted to the table and the spindle housing bolts to the top plate. The first load cell is made by JR3 and it is a strain-gage type 
Table 3.1: Stiffness of JR3 Load Cell

\begin{tabular}{|c|c|c|}
\hline \hline JR3 Direction & Global Direction & Stiffness, N/m or N m/rad \\
\hline$K_{x}$ & $K_{y}$ & $130 \mathrm{e} 6$ \\
$K_{y}$ & $K_{x}$ & $130 \mathrm{e} 6$ \\
$K_{z}$ & $K_{z}$ & $1.12 \mathrm{e} 9$ \\
$K_{M x}$ & $K_{M y}$ & $3.84 \mathrm{e} 6$ \\
$K_{M y}$ & $K_{M x}$ & $3.84 \mathrm{e} 6$ \\
$K_{M z}$ & $-K_{M z}$ & $1.20 \mathrm{e} 6$ \\
\hline
\end{tabular}

cell, with 6 axes of measurement output: the three force axes (x,y, and $z$ ) and the three moments about those force axes (moment about $x$, moment about $y$, and moment about $z$ ). Please see table 3.1 for quoted stiffness values in each direction. Physically the load cell is a "pancake", about an inch thick and 6 inches in diameter. The output passes through a JR3 strain gauge amplifier before being sampled by the data acquisition system. This system (spindle-JR3 load cell) had some very pronounced problems at a number of resonances in the 6,000 to $12,000 \mathrm{rpm}(100$ to $200 \mathrm{~Hz})$ range, and so in addition it was tested with some added damping material. The damping was accomplished by adding a thin layer (0.115" uncompressed) of 30-durometer Sorbothane material in parallel with the load cell, along two sides of the spindle. To bridge the rest of the gap between the aluminum fixture plates, a 1.5-inch aluminum boxbeam was used. Once the Sorbothane was compressed between the boxbeam and the upper aluminum fixture plate, it lost about $25 \%$ of its thickness, which is in the design range for the material.

The second load cell is made by Kistler (\#9257A) and it is a piezoelectric type, and hence much stiffer than the JR3. It measures force only, along each of the principal axes $\mathrm{x}, \mathrm{y}$, and $\mathrm{z}$. This load cell is designed for in-process measurement of machine tool forces in a manufacturing operation. The output from each channel goes to a Kistler 5004 charge amplifier before being sampled by the data acquisition system. Table 3.2 shows the quoted stiffness values for this load cell.

On the spindle rotor, to the rear of the chuck, I added an aluminum "balance ring." This ring fits over the rotor (figure 3-3) and is held there by two opposing set screws. Another two opposing tapped holes allow the placement of a third set screw, which is used as an imbalance mass. The balance ring has a tight (close to interference) fit around the rotor, so that moving the position of the ring has a minimal effect on radial location of the imbalance 
Table 3.2: Stiffness of Kistler Load Cell

\begin{tabular}{|c|c|c|}
\hline \hline Kistler Direction & Global Direction & Stiffness, N/m \\
\hline$K_{x}$ & $-K_{y}$ & $1 \mathrm{e} 9$ \\
$K_{y}$ & $-K_{x}$ & $1 \mathrm{e} 9$ \\
$K_{z}$ & $-K_{z}$ & $2 \mathrm{e} 9$ \\
\hline
\end{tabular}

Table 3.3: Imbalance Masses

\begin{tabular}{|c|c|c|}
\hline \hline Designation & Mass, $\mathrm{g}$ & Effective Radius, $\mathrm{cm}$ \\
\hline $\mathrm{A}$ & 0.4883 & 2.301 \\
$\mathrm{~B}$ & 0.8679 & 2.454 \\
$\mathrm{C}$ & 1.2273 & 2.625 \\
$\mathrm{D}$ & 1.9297 & 2.948 \\
\hline \hline
\end{tabular}

mass. Since the opposite tapped hole is empty, we know exactly the imbalance mass; if we assume the center of mass of the setscrew to be its geometric center, we know the radius at which the imbalance mass acts on the system. Using four different lengths of set screw allowed me four different masses, although for most of the experiments only the smallest mass (" $A$ ") was used. The balance ring can be loosened and rotated to any position on the rotor, which is useful for phase angle experiments: it allows any relative angle between imbalance and geometric decenter to be adjusted. Refer to table 3.3 for a description of the imbalance masses used.

The metrology ball was originally a stock Lion Precision ball (B014-5770) which was modified to fit in a 5/8-inch collet. This meant machining a new cylinder of steel, $5 / 8$ " in diameter with a 1/4" internal bore. The idea behind adjustment of the metrology ball center is a flexure shaft. Four setscrews, at 90 degrees to each other, put pressure on the flexure and in this way move the center of the ball around. (Refer to figure 3-2.) Our steel bore had an interference fit for the back half of the Lion flexure shaft, with about 0.001 " of radial travel for the shaft in front of the flexure. This enabled ball centering adjustments within a range of about 100 microinches. This setup was extremely repeatable test to test, holding a ball offset to within 5 microinches and maintaining the offset angle after high speeds and spindown. Using this ball, we were able to maintain a known geometric decenter or get the ball running true within a few microinches.

To measure the position of the metrology ball at rest and at high speeds I employed 


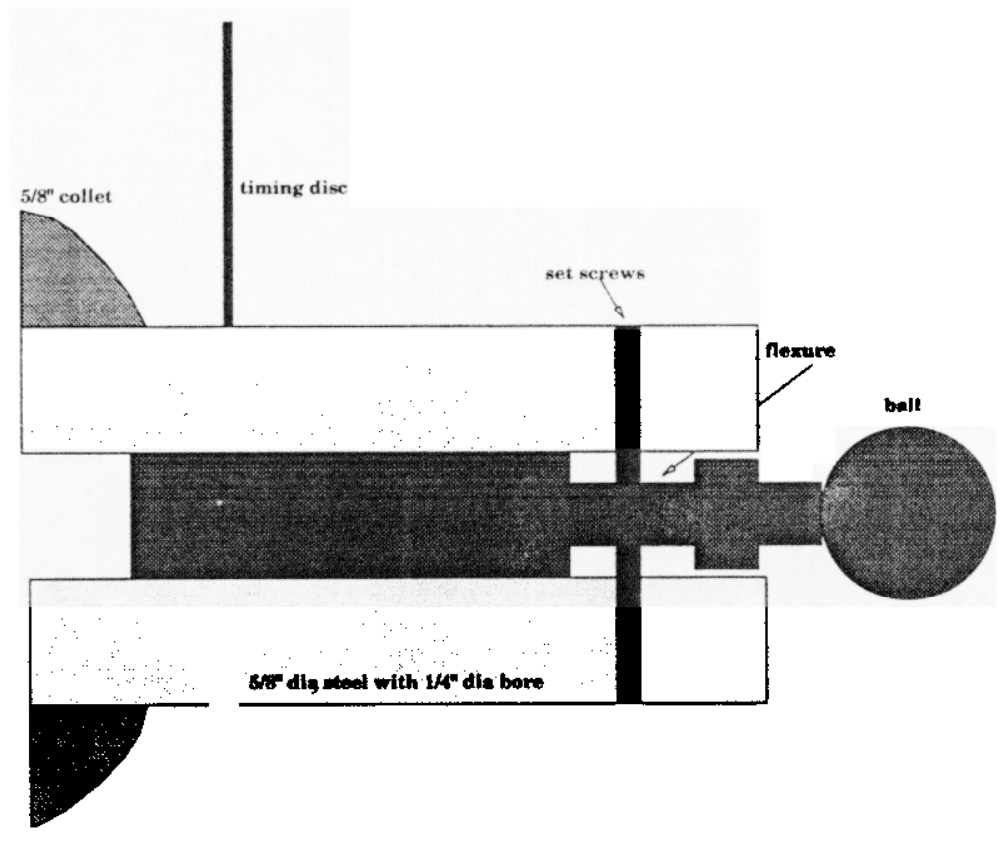

Figure 3-2: Cross-section of metrology ball, showing flexure shaft.

three orthogonal Pioneer capacitance gauges. These are mounted in an aluminum fixture that can be adjusted for the diameter of the ball. Two capacitance gauges look at the ball radially: one looks horizontally at the ball (labeled "Radial1") and the second looks vertically up at the ball ("Radial2"). The third capacitance gauge looks end-on at the ball ("Axial"). The fixture is clamped to an angle iron, which is toe-clamped to the granite block. (See figure 3-1.) By using a light screwdriver, the capacitance gauge fixture can be tapped so that the gauges are in range on the ball. Once in range, the clamps are locked down. Finer adjustment can be made using the offset potentiometers on the capacitance gauge amplifier. The amplifiers are also supplied by Pioneer, but in this application were integrated into one LLNL-designed cabinet. Each capacitance gauge amplifier has three ranges: full-scale 1000,100, and 10 microinches. For most of the experiments, the 100microinch full scale was used. The capacitance gauges were calibrated over their operating range using a Mituyo calibration tester (model 521-106), with a positioning resolution of 10 


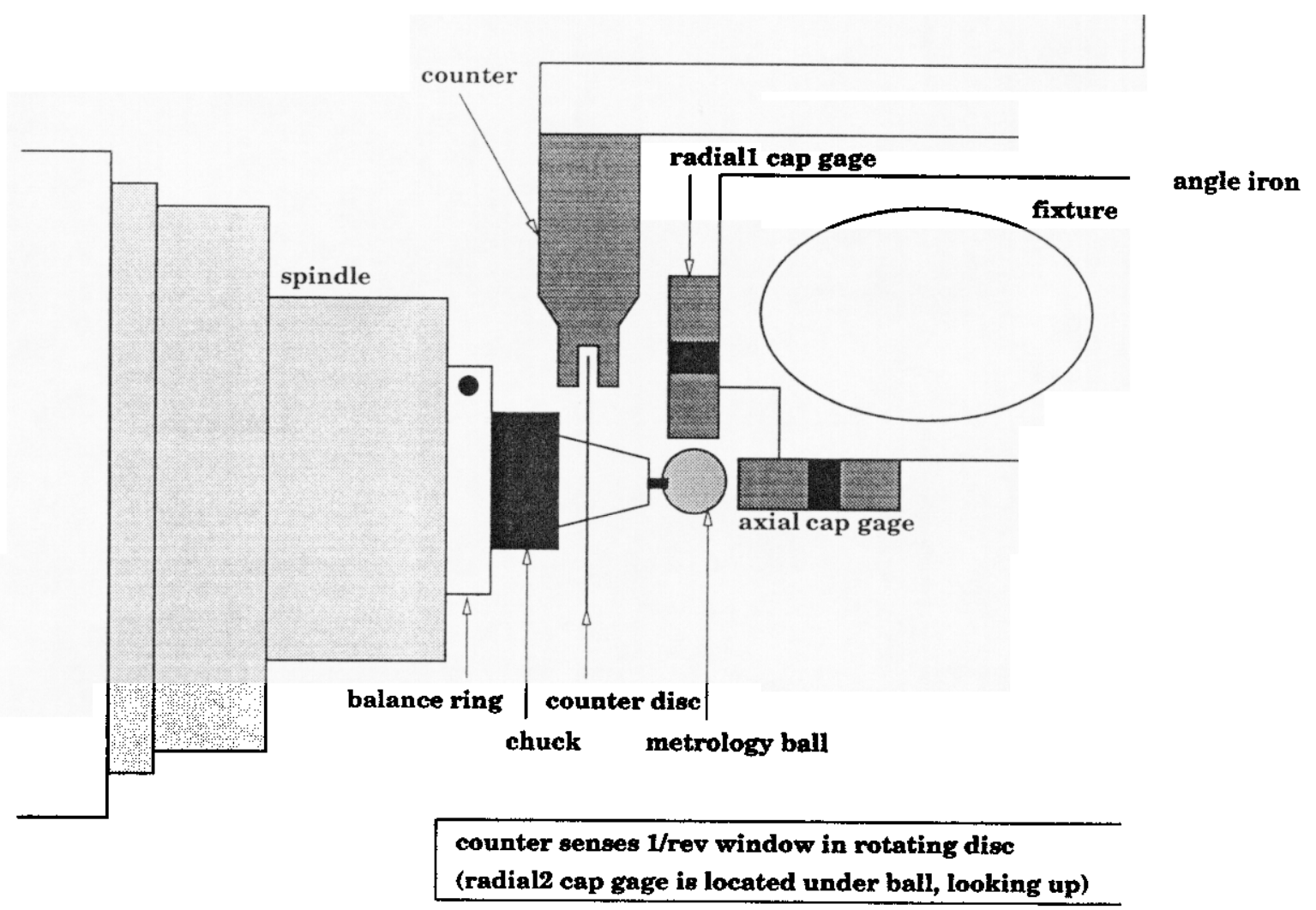

Figure 3-3: Top view schematic of spindle analyzer setup showing $1 /$ rev counter.

microinches, and a nanovoltmeter.

To measure the rotational speed of the shaft, a timing window and photoeye were used. As part of the metrology ball fixture, there is an aluminum disk with a 0.1 " window. The "counter", which sits alongside the Radiall capacitance gauge, has a photoeye connected to $a+5 / 0$ Volt logic gate. This provides a once-per-revolution pulse (see figure 6-1) which can be used both to determine the rotational speed and as an angle by which other parameters (e.g. imbalance forces and ball decenter) can be referenced. In general, 0 degrees refers to the start of the counter pulse. This setup can be seen in figure 3-3. 


\subsection{Data Acquisition}

Data acquisition for the Westwind spindle system was a challenge. In order to acquire data with the JR3 dynamometer, we needed 10 channels of synchronized input ( 6 from the load cell, 3 from the capacitance gauges, and 1 from the counter). This was accomplished using two different CPUs with three different A/D boards. The first CPU is a Tektronix 2510 8-channel dedicated data acquisition system. It acquires data at $25 \mathrm{kHz}$ per channel, with built-in $12.5 \mathrm{kHz}$ anti-aliasing filters. It has two A/D boards (labeled "lower" and "upper"). To acquire the data for the capacitance gauges, we use a Pentium PC with a 4-channel National Instruments $\mathrm{A} / \mathrm{D}$ board which samples at $25 \mathrm{kHz}$ per channel. The outputs from the Pioneer capacitance gauge amplifiers are first passed through a Stanford Research anti-aliasing filter (set at $12.5 \mathrm{kHz}$ ) and then sampled by the National Instruments A/D board. Each of the three A/D boards gets its own counter pulse signal, which is used post-acquisition to synchronize and reference the data. The data is saved to disk in ASCII form on the Pentium, but a fairly elaborate process must be used to extract the data from the Tektronix system. Figure 3-4 shows a flowchart for data acquisition with the JR3 dynamometer.

Starting the two CPUs at the same instant for the acquisition was a challenge. This was accomplished with a lot of help from and work by a data acquisition specialist. Essentially, the Pentium system waits for the Tektronix to begin acquisition, and then starts its own, within one clock pulse (40 microseconds) of the Tektronix starting. This works using a "trigger out" feature of the Tektronix. The Tektronix has a "trigger out" that goes from 0 to +5 Volts when the acquisition starts. This trigger pulse is coupled through an AND logic gate with the clock on the Pentium A/D board. The Pentium A/D waits for its own clock to start to signal the beginning of its data acquisition, and this cannot happen until the Tektronix has started. Refer to figure 3-5 for a schematic of the synchronization setup. This was implemented using a dedicated $\mathrm{C}$ program which ran in the PC. It should be noted here that there is a potential problem with discrepancy between the clock speeds of the two systems: this can be sorted out after the data has been collected. The counter pulse serves as a time reference to sychronize the two datasets during data analysis. (A better solution would have been to use a calibrated frequency generator as a reference pulse. However, lack of extra input channels prevented this in our case.) 
Pioneer cap gage

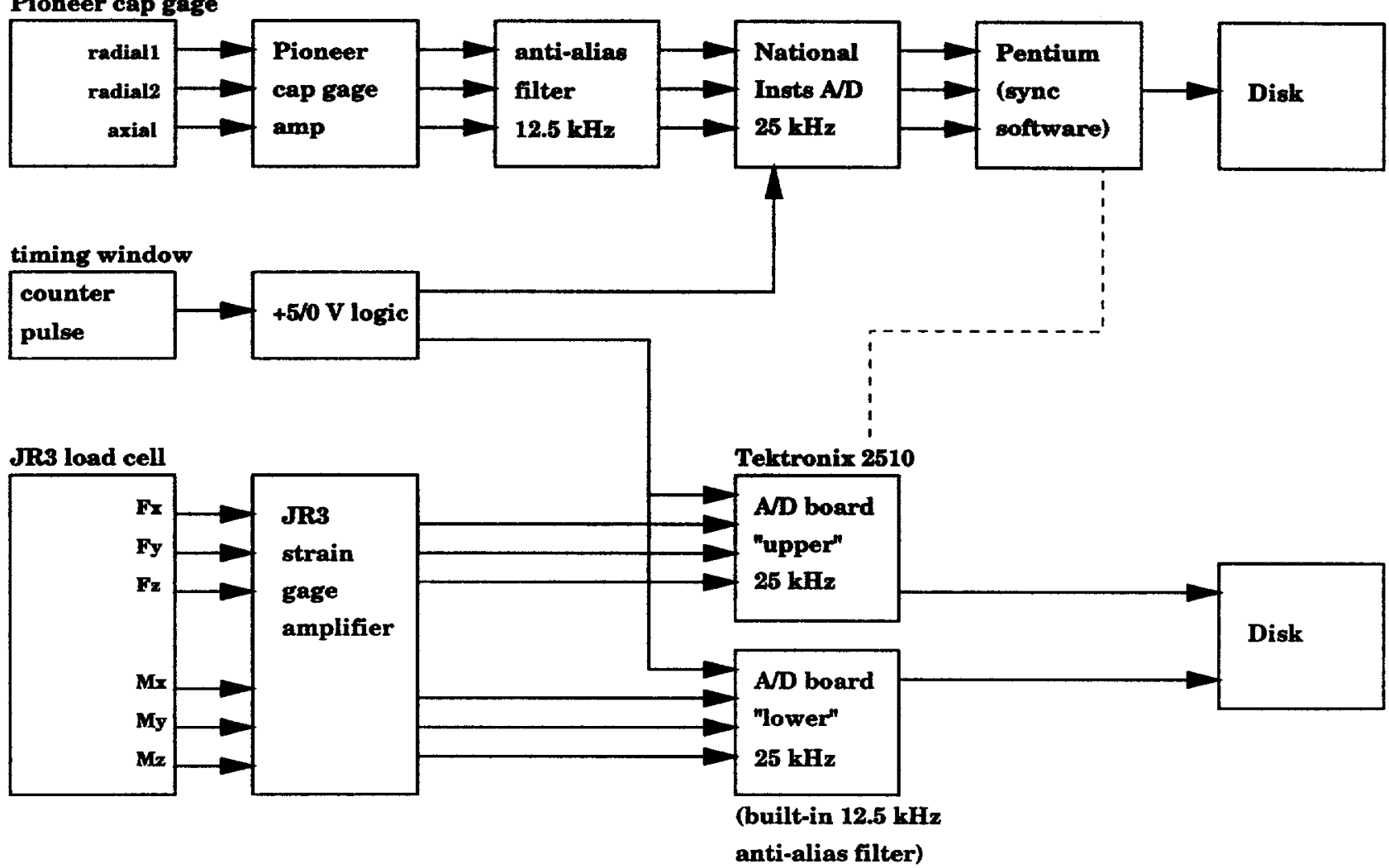

Figure 3-4: Flowchart for 12-channel data acquisition with JR3 dynamometer. 
In addition to performing startup synchronization of the two systems, the PC program had a graphical interface to monitor capacitance gauge position and spindle speed and a direct memory access (DMA) routine to record data. The DMA ran in the background and could take data indefinitely. It did this by writing data to the hard disk every time half of its allocated buffer was filled. By the time the second half of the buffer filled, the DMA was ready to overwrite the first half, and this process could continue until the hard disk filled.

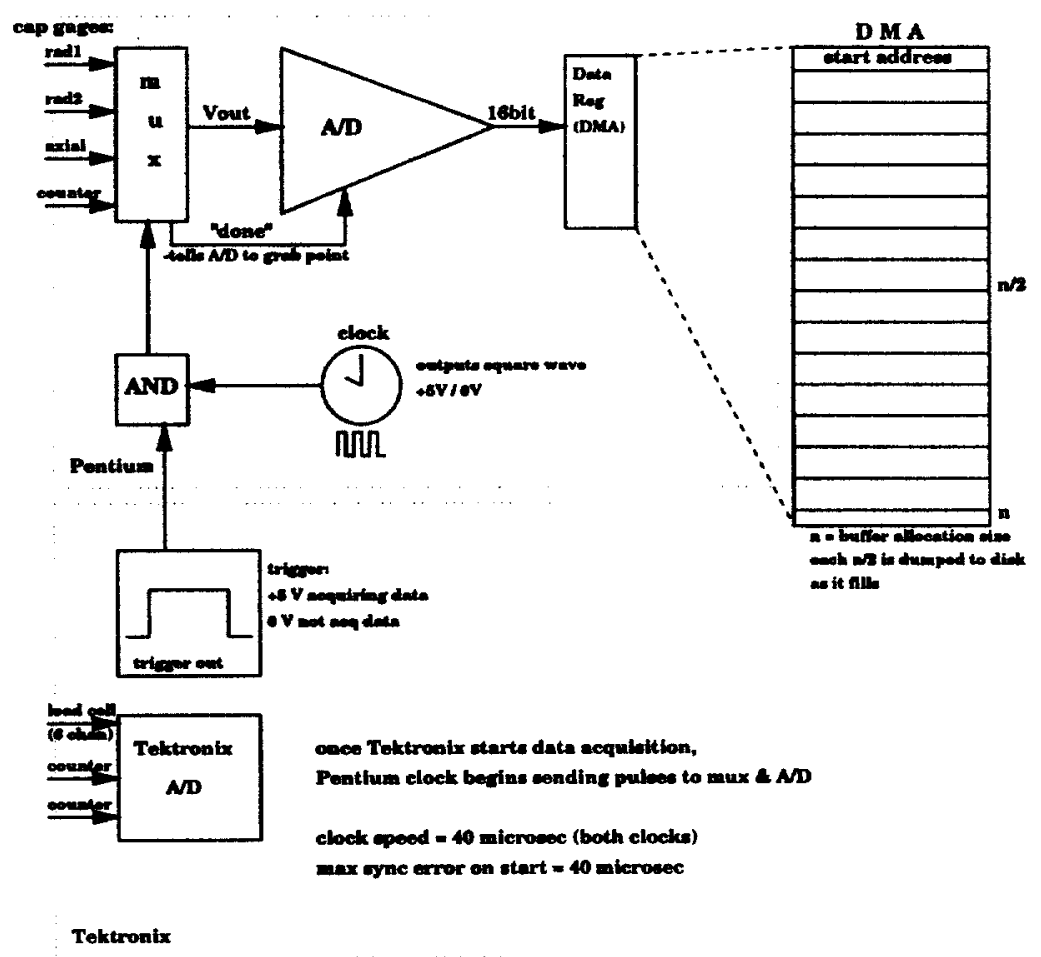

Figure 3-5: Synchronization scheme for 12-channel data acquisition using two CPUs.

The reason we did not use a frequency generator for synchronization of the two systems was simply a lack of channels. In order to take rotational speed data, capacitance gauge data, and force data from the JR3 load cell we needed to use our maximum channel capacity of 12 channels (the ten referred to above plus two additional channels for synchromization across the three A/D cards). As a compromise, we used the once per revolution counter pulse as a synchronization pulse, even though this has the disadvantage of slowing down in frequency over the course of the acquisition time (one second). This is because the spindle drifts down in speed approximately $1 \%$ over that time period. 
In the case of the Kistler setup, data acquisition was much simpler. This required only 7 channels of input ( 3 for the Kistler outputs, 3 capacitance gauges, and counter pulse). All 7 channels could be acquired on the Tektronix 2510, with an additional counter channel to synchronize data from the two A/D boards. There was no need to synchronize two systems. The graphical interface on the PC could still be used to track ball position and rotational speed, although the PC was not used to acquire any data. Figure 3-6 gives a flowchart for the Kistler data acquisition.

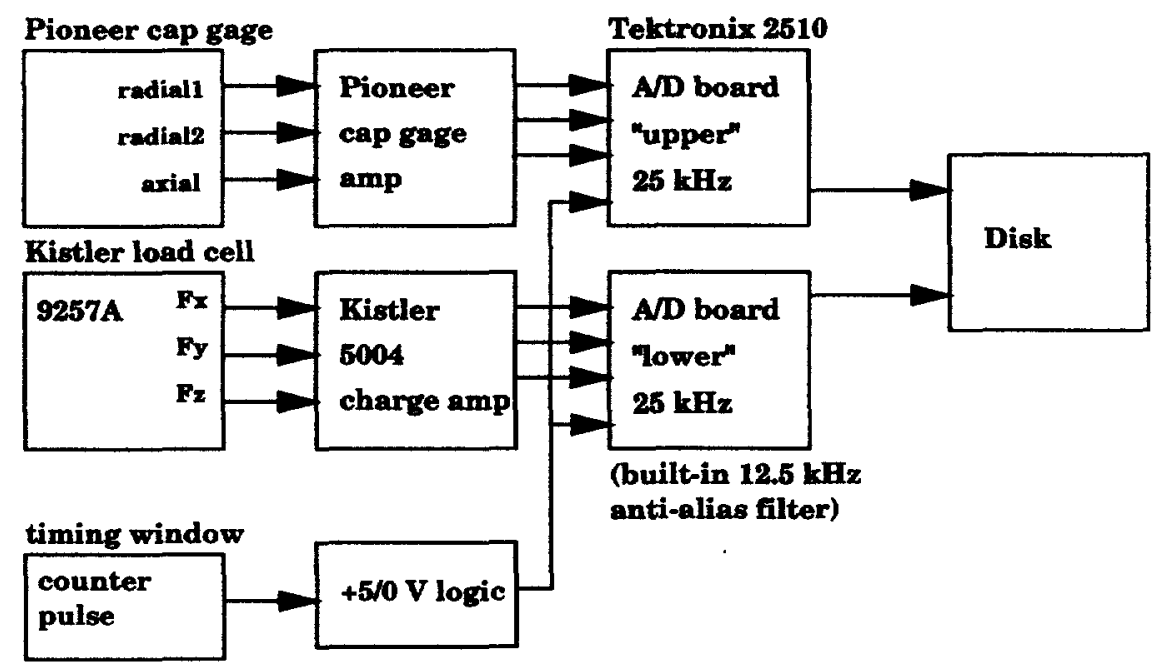

Figure 3-6: Flowchart for 8-channel data acquisition with Kistler dynamometer. 


\section{Chapter 4}

\section{Empirical Modal Analysis}

This chapter discusses empirical modal analysis, which was performed on each of the three systems: Westwind-JR3, Westwind-JR3-damping, and Westwind-Kistler, as well as a fourth system, the Westwind spindle bolted directly to the granite tabletop. Modal analysis yielded fundamental insight into the dynamics of the spindle system.

\subsection{Procedure}

Empirical modal analysis works by measuring structural response to a force input at a series of locations ("nodes"). Then the frequency responses at each node are combined to yield information on the mode shapes at each frequency. To do this in practice, the structure is instrumented with three-axis accelerometers at each desired node and excited with an instrumented hammer. A spectrum analyzer takes the time signal from each accelerometer and the hammer and converts it into a frequency response spectrum. Knowing the input and output frequency responses allows determination of a numerical transfer function between the excitation point and each node, in each direction. A software package then takes these "frequency response functions" and knowledge of the location of the nodes (the "mesh") and curvefits the frequency response functions to yield mode shapes for the structure. Alternately, modal analysis may be performed by instrumenting the structure with an accelerometer in one location and exciting the structure in multiple locations: the two processes are mathematically equivalent, although for many systems it is easier to instrument small accelerometers at each node than it is to get a much larger hammer to strike at each node. . 
For the modal tests presented here, we used STAR modal analysis software, which is a commercially-available empirical modal analysis package. The STAR software imports frequency spectra from the spectrum analyzer (Hewlett Packard 3566A multi-channel analyzer) and does one of several curvefit routines to determine the empirical modal frequencies and mode shapes. It should be noted here that modal analysis is not an exact science; it involves a fair amount of experienced decision-making on the part of the person conducting the test and analyzing the data. For example, a few frequency spectra from a system may show a small "bump" at the same frequency (in contrast to high, clean peaks at other frequencies). Whether or not this small bump is worth curvefitting as its own mode is entirely up to the experimenter. Mode shape data from such low peaks is often so deep "in the noise" that useful results are often impossible to extract. In practice, the experimenter usually curvefits anything remotely promising, and then later decides which modes are due to real system oscillation and which are relics of noisy data acquisition.

For this system, we instrumented 33 points on and around the spindle structure with Endevco model 63-500 three-axis accelerometers. These points included four on the underlying granite block (at each corner of the mounting plates), four on the aluminum plate under the load cell, four on the aluminum plate above the load cell, eight on the spindle housing, eight on the spindle tailstock, four on the headstock, and one on the rotor (at the balance ring location). Figure 4-1 shows the mesh used in the tests of the Westwind spindle mounted to a JR3 dynamometer. Each intersection of lines on the mesh represents a node, or accelerometer placement location. The large cone is the front of the spindle, with the cone vertex representing the location of the balance ring on the rotor. The box in the center is the spindle housing, while the left-most four points are locations on the spindle tailstock. At the bottom are the four points on the granite table and above those, the four points on the bottom aluminum plate. Another square of four points above those represents the top aluminum plate. In between is the dynamometer. The mesh used on the Kistler and no load cell tests is similar to the one in figure 4-1.

The frequency response was measured at each point by a three-axis accelerometer. The spindle was excited on the housing with a Dytran 5801A3 instrumented hammer, at a 45degree angle to the $\mathrm{Y}$ and $\mathrm{Z}$ axes, near the joint between housing and spindle. This point was chosen as it would excite both the housing modes and the spindle modes, in two of the three orthogonal directions. Each frequency response function output by the spectrum 


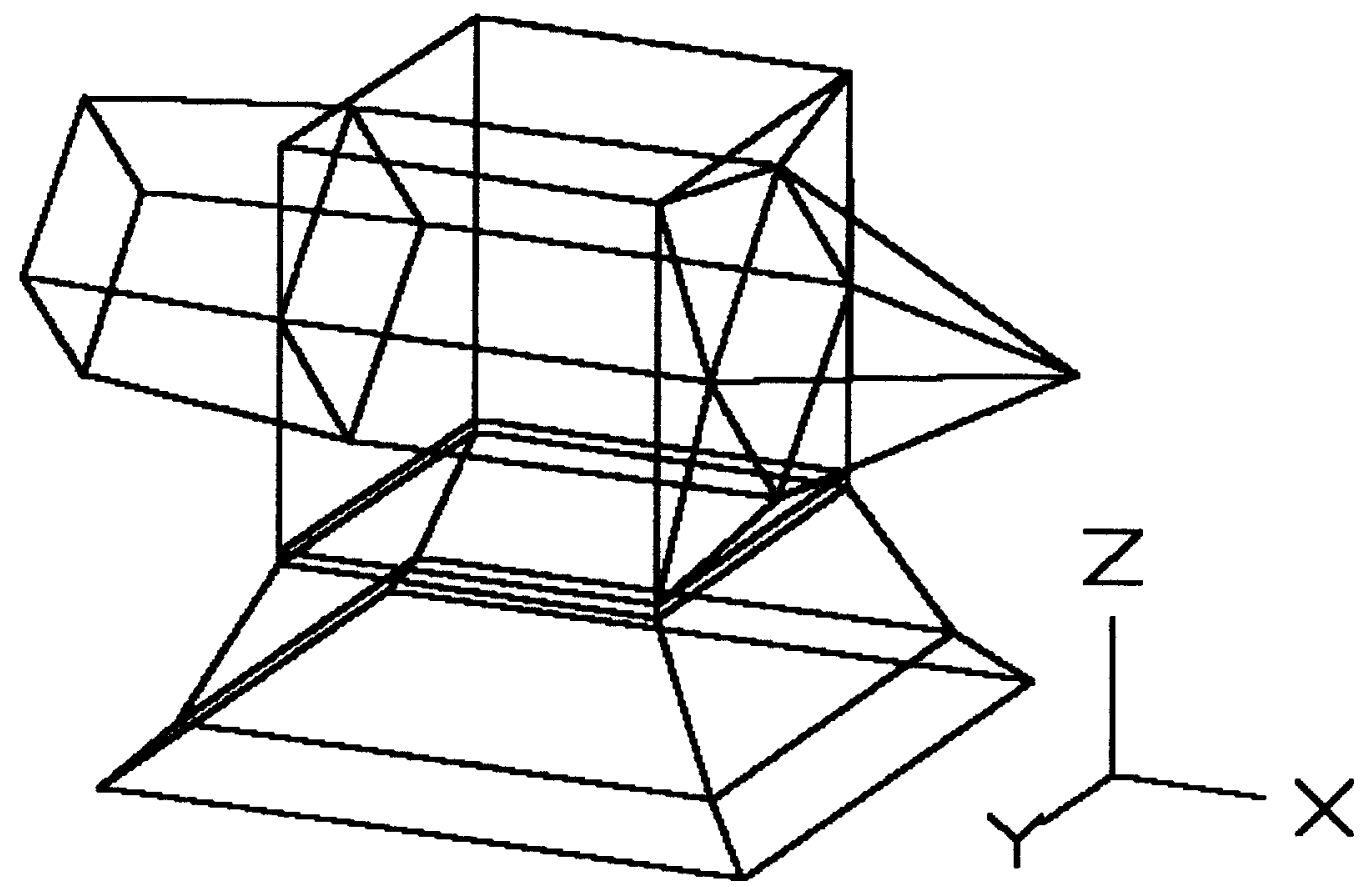

Figure 4-1: Mesh used in STAR JR3 tests. 
analyzer was actually the average of ten hammer strikes, performed in-process by the HP spectrum analyzer.

After data importation into STAR and curvefitting, the software allows the user to look at an animated picture of the motion of the structure at each calculated natural frequency. This is extremely useful as it gives the user an intuitive (and exaggerated) picture of how the structure is moving at each frequency. The software also provides tables of magnitude and phase of the motion of each node at each natural frequency. In addition, the software enables simulation of response to a driving sinusoid (or any arbitrary function) at any one of the nodes. This feature was useful, as it enabled a computational model of system response to an imbalance driving function.

\subsection{Results}

The empirical modal analysis testing produced results which are central to this project. It showed that the spindle-load cell system was essentially oscillating in a rigid-body fashion, which enabled construction of an analytical rigid-body model for spindle oscillation. By rigid-body, we mean that the entire spindle structure, housing included, moves rigidly about the compliant load cell with respect to the granite table. Most of the oscillatory modes involved the spindle structure as a whole rotating with respect to the granite table, often about the center of the load cell. In some cases, the center of rotation was somewhere else on the spindle, giving the impression of combined translational and rotational motion (although for purposes of the analytical model, this was combined into rotational motion about the stationary point).

In addition, there were other useful results: the base of the system does not move appreciably with respect to the granite table to which it was bolted. However, there is some appreciable "slip" between the spindle housing and the aluminum plate to which it is bolted at some frequencies. This is probably due to the addition of (low-friction) circuitboard material between the housing and the plate, which cuts down on an electrical noise problem (which did not turn out to be "noise"; see the chapter on "Experimental Challenges"). In addition, at some frequencies the tailstock of the spindle moves relative to the housing (and 180 degrees out of phase). This is an expected effect; according to Westwind, they have received customer feedback about a resonance between their spindle and housing at 
some rotational speeds. They call the effect "belling", the ringing of the spindle (which is attached only at the front of the housing) inside its housing. At some frequencies there is noticeable axial motion of the spindle rotor. Refer to the tables presented in this chapter for the numerical results of each modal test and descriptions of the global mode shapes.

\subsubsection{Westwind-JR3 system}

Table 4.1 shows the modal analysis results for the JR3 system without damping material. In these tables, the damping value in $\mathrm{Hz}$ refers to the width of the peak in $\mathrm{Hz}$ at the half-amplitude point on the peak. In all cases the peak is very narrow; there is very little damping in the load cell. The two curvefits ("normal" and "advanced") refer to different algorithms used within the STAR software to calculate modal peaks. It is often useful to run several of the available algorithms on the raw spectrum analyzer data; often one curvefit algorithm will pick up on a mode that another missed. Some of the algorithms are tailored to find "local" modes; others find "global" modes of the whole structure.

The first four modes of this system are all rigid-body modes: predominantly the spindle rotating about the center of the load cell in one of the three rotational degrees of freedom. Refer to figures 4-2, 4-3, and 4-4 for STAR-generated pictures of these modes. The figures show the maximum displacement of the structure at both ends of its travel. (They are taken from the STAR results animation of these modes.) Of course, the amplitudes of oscillation are greatly exaggerated for purposes of visualization.

The higher-frequency modes are more complex, and involve combinations of translation and rotation of the spindle structure with relation to the load cell. (An example of this can be seen in figure 4-5, the mode at $289 \mathrm{~Hz}$, where the spindle translates in the Z-direction and rotates about $\mathrm{Y}$ at the same time.) At $323 \mathrm{~Hz}$ there is a great deal of translation in the Z-direction and simultaneous rotation about the $Y$ direction. The highest mode, at 464 $\mathrm{Hz}$, has the spindle twisting about its centerline, with the rotor moving axially at the same time.

With the addition of damping material, not very much changes in the STAR results. Table 4.2 presents the results of the modal tests on the JR3 plus damping material system. The first mode drops slightly in frequency, while the highest raises in frequency, but both of these changes are relatively minimal. Nor does the peak width (damping) change appreciably between the two tests. Most of the data is very similar between the undamped and 
Table 4.1: JR3, no damping: natural frequencies and mode shape descriptions. *-see related figure

\begin{tabular}{|c|c|c|c|c|l|}
\hline \hline & \multicolumn{2}{|c|}{ Normal Curve Fit } & \multicolumn{2}{|c|}{ Advanced Curve Fit } & \\
\hline Mode no. & Freq, Hz & Damp, Hz & Freq, Hz & Damp, Hz & Description \\
\hline $1^{*}$ & 96.7 & 1.5 & 97.5 & 3.1 & rigid-body about Y axis, load cell \\
$2^{*}$ & 113.3 & 1.4 & 113.2 & 1.5 & rigid-body about X axis, load cell \\
3 & 128.8 & 0.7 & & & rigid-body about X axis, LC, \\
& & & & & some tail shake about Z \\
$4^{*}$ & 163.3 & 2.7 & 163.3 & 2.8 & rigid-body about Z axis, LC \\
$5^{*}$ & 289.5 & 2.3 & 288.9 & 7.7 & Z dir trans, some My also \\
6 & 323.2 & 7.5 & 323.7 & 7.4 & Z dir trans \\
7 & 464.3 & 2.1 & 463.8 & 4.5 & twist about CL of spindle, \\
& & & & & some motion of rotor in axial dir \\
\hline
\end{tabular}
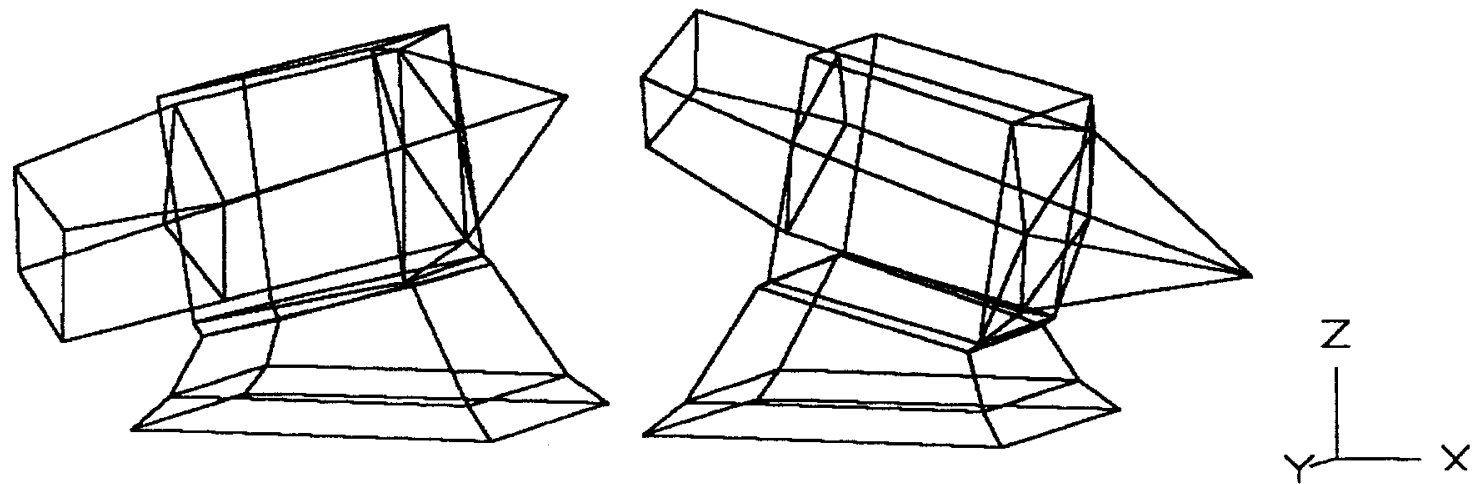

Figure 4-2: Undamped JR3 system, mode 1, 96.7 Hz. Rotation about $\mathrm{Y}$ axis of load cell. 

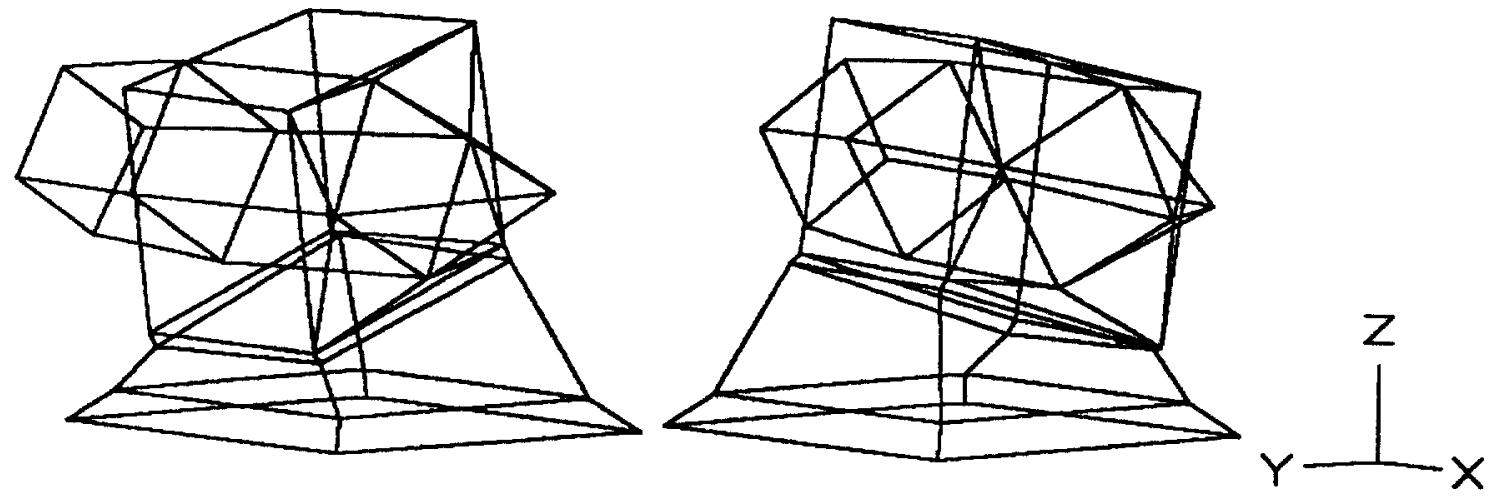

Figure 4-3: Undamped JR3 system, mode 2, 113.3 Hz. Rotation about X axis of load cell.

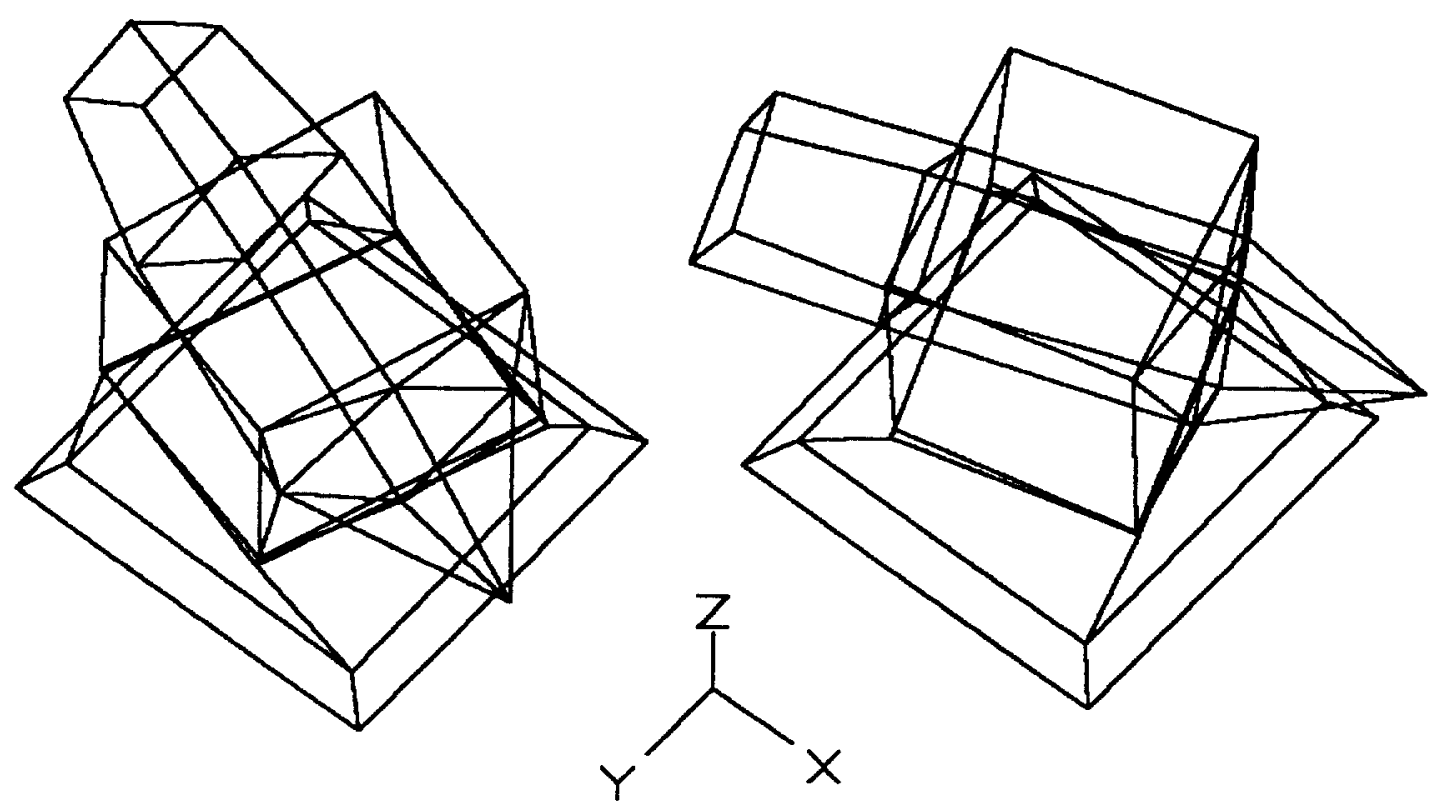

Figure 4-4: Undamped JR3 system, mode 4, $163.3 \mathrm{~Hz}$. Rotation about $\mathrm{Z}$ axis of load cell. 

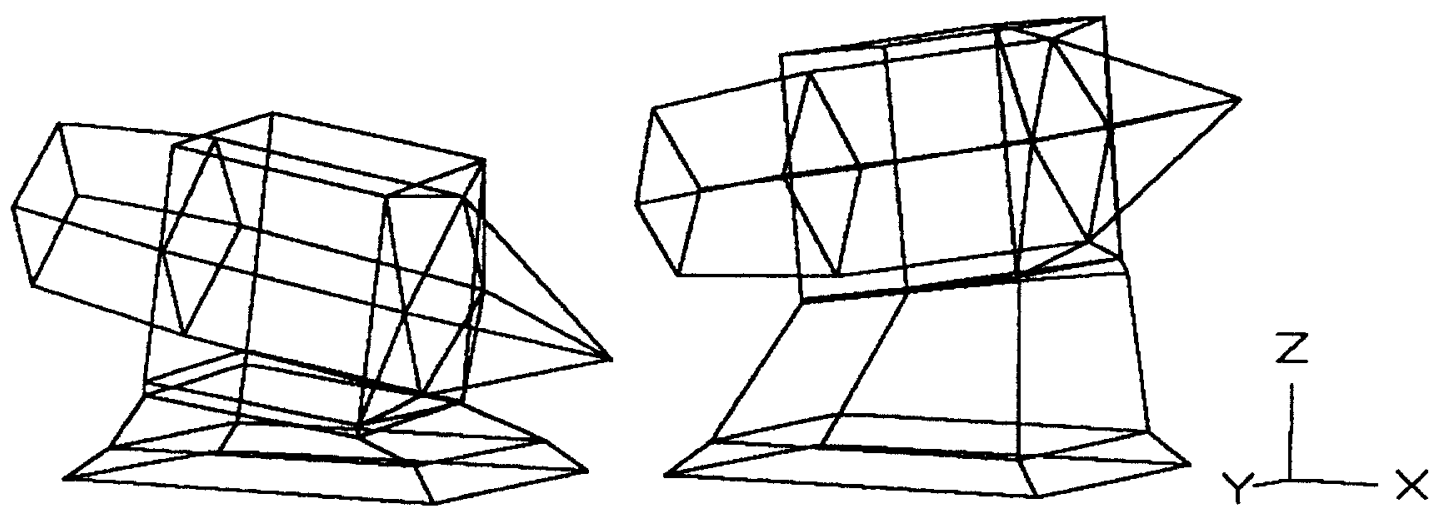

Figure 4-5: Undamped JR3 system, mode 5, $289.5 \mathrm{~Hz}$. Combined translation and rotation.

Table 4.2: JR3 with damping: natural frequencies and mode shape descriptions

\begin{tabular}{|c|c|c|c|c|l|}
\hline \hline & \multicolumn{2}{|c|}{ Normal Curve Fit } & \multicolumn{2}{|c|}{ Advanced Curve Fit } & \\
\hline Mode no. & Freq, $\mathrm{Hz}$ & Damp, Hz & Freq, Hz & Damp, Hz & Description \\
\hline 1 & 93.9 & 2.0 & 93.9 & 2.1 & rigid-body about Y axis, load cell \\
2 & 123.0 & 2.3 & 122.7 & 2.6 & $\begin{array}{l}\text { rigid-body about X axis, LC, } \\
\text { some tail shake about Z } \\
\end{array}$ \\
& 130.0 & 1.5 & & & similar to 2 \\
3 & 165.6 & 3.9 & 164.5 & 4.6 & rigid-body about Z axis, LC \\
4 & & & 167.5 & 3.0 & Z direction translation \\
5 & 289.3 & 1.2 & 289.5 & 5.5 & Z dir trans, some My also \\
6 & 320.9 & 8.8 & 321.2 & 8.1 & similar to 6 \\
7 & 491.1 & 0.2 & & & spindle shaft moving axially, \\
8 & & & & & some Z trans \\
\hline \hline
\end{tabular}

damped JR3 systems. This is probably because adding the damping material in this case mainly serves to reduce the amplitudes of the resonant oscillations; the system itself is not fundamentally changed. If the system had been significantly modified in the addition of damping, more drastic changes in the mode shapes could be expected.

\subsubsection{Westwind-Kistler system}

Table 4.3 shows the modal analysis results for the Westwind-Kistler system. The fact that this system is stiffer than the JR3-Westwind system is readily apparent. The first mode occurs at $127 \mathrm{~Hz}$, as compared to 97 for the JR3 system. Also, there are fewer fundamental 
Table 4.3: Kistler: natural frequencies and mode shape descriptions

\begin{tabular}{|c|c|c|c|c|l|}
\hline \hline & \multicolumn{2}{|c|}{ Normal Curve Fit } & \multicolumn{2}{|c|}{ Advanced Curve Fit } & \\
\hline Mode no. & Freq, Hz & Damp, Hz & Freq, Hz & Damp, Hz & Description \\
\hline 1 & 127.0 & 1.5 & 139.0 & 3.2 & $\begin{array}{l}\text { rigid-body about X axis, load cell } \\
\text { rigid-body about X axis, load cell, } \\
\text { rotor moves clockwise about axis } \\
\text { of rotation } \\
\text { rotation about Z axis, LC, } \\
\text { tail motion slightly lags housing } \\
\text { pure Z translation } \\
\text { large axial motion of rotor, } \\
\text { Z dir trans, some rot. about Y, LC }\end{array}$ \\
\hline
\end{tabular}

modes - only five. The Kistler frequency response data was subjectively much cleaner that that for the JR3; the modal peaks were more pronounced and there were less questionable peaks "in the noise." The first three modes are of the spindle about the load cell principal axes: $\mathrm{Y}, \mathrm{X}$, and $\mathrm{Z}$. At higher frequencies, we see an almost pure translation in the Z-direction at $412 \mathrm{~Hz}$ and at $464 \mathrm{~Hz}$ a large motion of the rotor against its thrust bearings (axially).

\subsubsection{Westwind (no load cell)}

Finally, the fourth modal test performed looks at the spindle by itself, bolted to the granite block. This data can be seen in table 4.4. This structure by itself (no load cell) is extremely stiff: one way to tell is to look at the relative motion between nearby nodes. On the earlier tests (with load cell) the nodes mostly move together, in a rigid fashion. On the tests of the spindle alone, however, there is sometimes great motion between neighboring points (i.e. a twist in the housing at the first mode, which was never observed with a load cell in place). This suggests that nothing is moving very much at all for these tests! One of the modes observed here, $457 \mathrm{~Hz}$, is similar to the highest seen on the JR3 and Kistler tests (both at $464 \mathrm{~Hz}$ ): the motion of the rotor of the spindle in an axial sense is a component of the modes of all three systems at this frequency. This suggests that this mode is "load cell independent" and derives from the spindle itself. Refer to figure 4-6 for a picture of this mode on the system with no load cell.

Many of the other modes in the spindle structure by itself seem to be lost when the 
Table 4.4: Spindle natural frequencies and mode shape descriptions (no load cell) *-see related figure

\begin{tabular}{|c|c|c|c|c|c|}
\hline & \multicolumn{2}{|c|}{ Normal Curve Fit } & \multicolumn{2}{|c|}{ Advanced Curve Fit } & \\
\hline Mode no. & Freq, $\mathrm{Hz}$ & Damp, $\mathrm{Hz}$ & Freq, $\mathrm{Hz}$ & Damp, $\mathrm{Hz}$ & Description \\
\hline 1 & 246.1 & 0.3 & & & tail moves in $\mathrm{Z}$ direction \\
\hline 2 & 301.8 & 6.3 & 297.8 & 10.5 & similar to 1 \\
\hline 3 & 317.5 & 0.4 & & & twist of housing about $\mathrm{Z}$, \\
\hline 4 & 429.8 & 1.0 & 425.4 & 12.3 & $\begin{array}{l}\text { twist of housing about } \mathrm{Z}, \\
\text { tail in phase w/ housing, } \\
\text { tail rotates about CL of spindle, } \\
\text { rotor moves axially }\end{array}$ \\
\hline $5^{*}$ & 456.5 & 5.0 & 455.9 & 7.0 & rotor moves axially \\
\hline 6 & 474.7 & 12.8 & 478.6 & 18.5 & $\begin{array}{l}\text { similar to } 3 \text {, } \\
\text { except tail in phase } w / \text { housing }\end{array}$ \\
\hline 7 & 696.0 & 14.7 & & & $\begin{array}{l}\text { translation in } \mathrm{Z} \text { and } \mathrm{X} \text { directions, } \\
\text { rotor moves in } \mathrm{Z} \text { direction }\end{array}$ \\
\hline 8 & 752.8 & 15.3 & 745.2 & 21.3 & similar to 7 \\
\hline
\end{tabular}

spindle is coupled to a load cell: this makes sense if these local modes are small in relative amplitude. Add a stiff system on top of a compliant spring and the modes involving the compliant spring will dominate the oscillations.

The modal data presented here was very helpful in this experiment. It allowed us to understand the shapes of the resonances we observed in our early spindle tests. Later, the modal data allowed us to model the structure and make predictions about spindle error motion based on system resonances. 

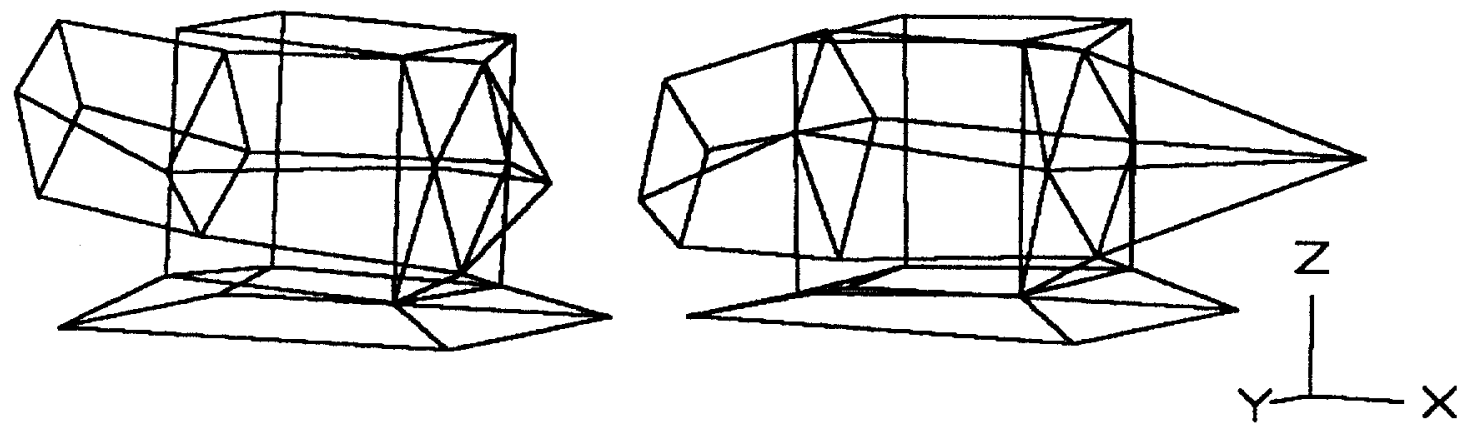

Figure 4-6: System with no load cell, rotor moving axially, $456.5 \mathrm{~Hz}$. 


\section{Chapter 5}

\section{Models}

\subsection{General Model}

\subsubsection{Description}

This section details the model presented at the ASPE conference in 1996 [1]. The model was used to check assumptions about the relevant grinding parameters given an imbalance and ic offset as inputs. The model allowed us to compare the relevance of different machine parameters in precision grinding. The model is schematically shown in figure 5-1.

This model is not meant to be applied to the real system investigated in this thesis. Rather, it looks at a large machine tool in a dynamic sense, in one dimension. For the model I will describe, it is important to note that only time-varying terms are considered. That is, constant-infeed cutting force is not considered, nor is cutting displacement. One should think of the model as providing insight into the error motions and oscillating forces about the process operating points. The model looks at small oscillatory behavior surrounding a large constant offset.

There are two inputs to the system: the imbalance force which is an oscillating sinusoid in one degree of freedom input to the grinding wheel mass, and a geometric decenter of the grinding wheel which is added as a force term acting between the grinding wheel mass and the workpiece rotor mass. This second input will be described in more detail below. In this model, we consider three resultant forces: the workpiece spindle force, the cutting process force, and the grinding spindle force. Workpiece and grinding spindle forces are the forces transmitted through the spring-dashpots connecting these spindle masses to the rest of the 


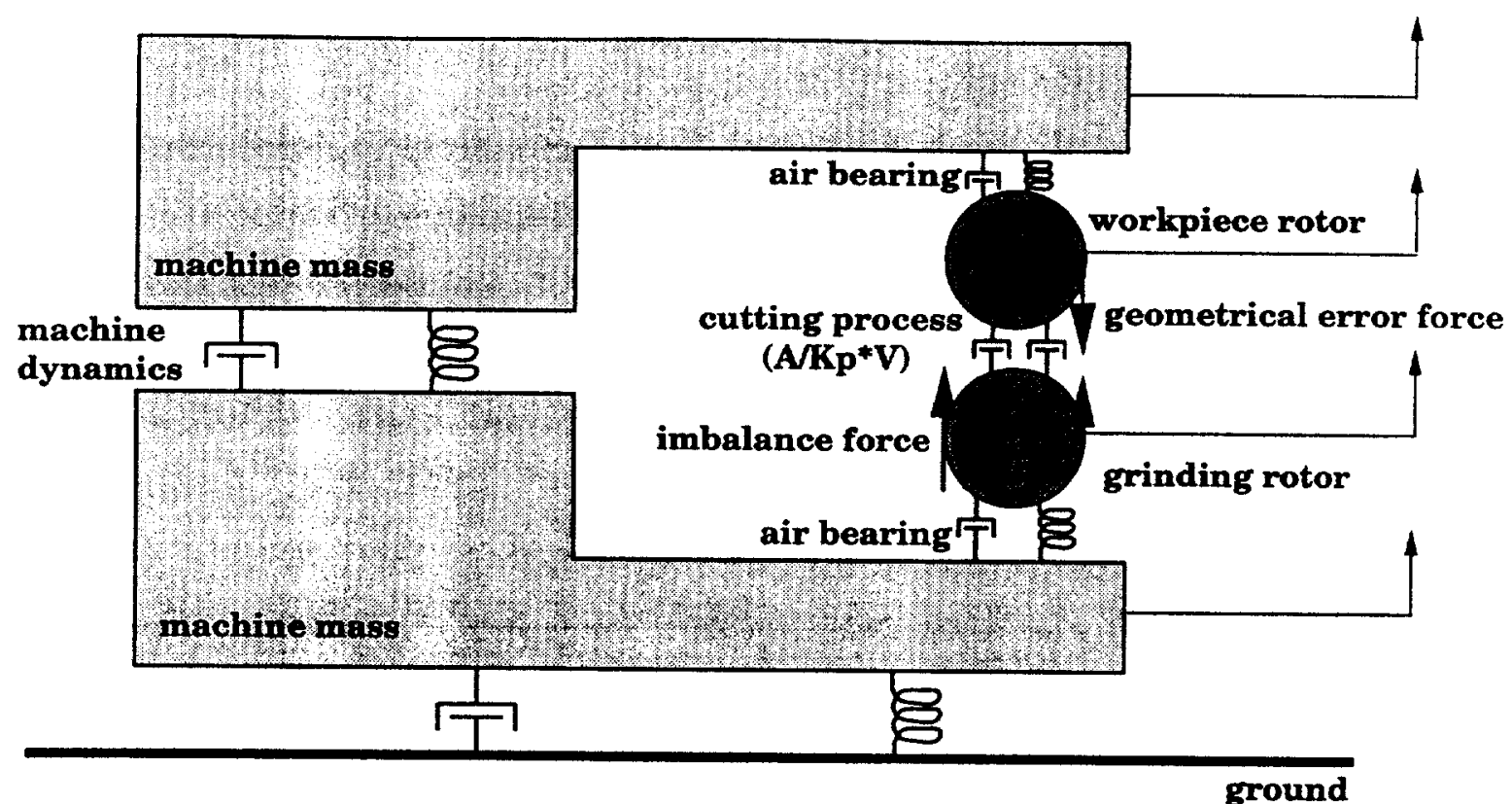

Figure 5-1: Dynamic grinding model.

machine tool. In a real machine, these correspond to the force "seen" by the air bearing. Cutting process force is the force between the spindles, felt by the workpiece.

At the heart of the model is a cutting process. For this model, I used a cutting model proposed by Thomas Kurfess in [8]. According to Dr. Kurfess, "The harder you push, the faster you grind," [10] and so the cutting process looks like a dashpot for small displacements. Putting Dr. Kurfess' statement another way,

$$
F=\frac{A}{K_{p} V} \dot{x}
$$

where $F$ is the cutting process force, $A$ is the surface area of the contact patch, $V$ is the relative velocity between cutting wheel and workpiece, and $\dot{x}$ is the infeed velocity. $K_{p}$ is Preston's coefficient, which is process-dependent and usually determined empirically. Thus, a model for the grinding process looks like a dashpot with damping constant $B_{p}$ equal to $\frac{A}{K_{p} V}$. In practice, we determined $B_{p}$ from a spark-out test: we let the wheel $\mathrm{d}$ well in one spot and watched as process force decayed to zero. The rate of decay is proportional to $e^{\left(-K_{\text {loop }} / B_{p}\right) t}$, where $K_{\text {loop }}$ is loop stiffness of the grinding machine, measured in a static test. In this way force oscillations due to imbalance on the grinding wheel play into 
velocity oscillations through the cutting model. Also, the grinding interaction is modeled as providing damping to the machine.

Separately, I wanted to consider the effect of arbitrary geometric eccentricities on the system. One of the central tenets of this model was the separation of effects due to pure imbalance and pure geometric eccentricity. An example of a geometric eccentricity would be an out-of-center grinding wheel or a wheel with lobes on the surface. (As opposed to an imbalance which imposes a pure force on the system.) In order to input this to the model, I considered the case of a pure displacement of the wheel, and its effects on the cutting process. A time-varying displacement $x$ would have velocity $\dot{x}$. This velocity would then couple into the cutting process equation to yield a force, since $F=B_{p} \dot{x}$. In this way an arbitrary velocity can be input to the model as an additional cutting process force acting on both masses: the grinding rotor and the workpiece rotor. In my implementation of the model, I used a simple wheel decenter as the velocity input; this looks like a sinusoid with a period of once per revolution of the grinding wheel. Using this method, one can phase-shift one input force relative to the other, to consider cases where the effects of imbalance and decenter happen separately. Of course, one input may be set to zero to consider cases where the imbalance or decenter dominates.

Each mass, the workpiece rotor and the grinding wheel, is separately sprung via simple air bearing models to the larger mass of the machine. Originally, this machine mass was one continuous element that surrounded the cutting elements, but later some simple machine dynamics (a spring-dashpot) were added to make the model more complete. In addition, the entire machine is separated from ground by a spring-dashpot, to simulate the effects of isolation.

This model evolved over time from a very simple two-mass (workpiece and grinding wheel) model to the one presented here. Once the graphical representation was worked out, I derived a differential equation for each mass, using $\Sigma F=m a$. The total forces acting on each mass came from the spring and dashpot equations which relate force to relative displacement or velocity, respectively. For this four degree-of-freedom model, I used MATLAB to simulate the response of each mass to force inputs. An example MATLAB script can be found in Appendix A. This script was used with the latest (unreleased) MATLAB ODE solvers, which allow better specification of error tolerancing and script specification of simulation parameters, among other improvements [16]. (The new solvers are available 


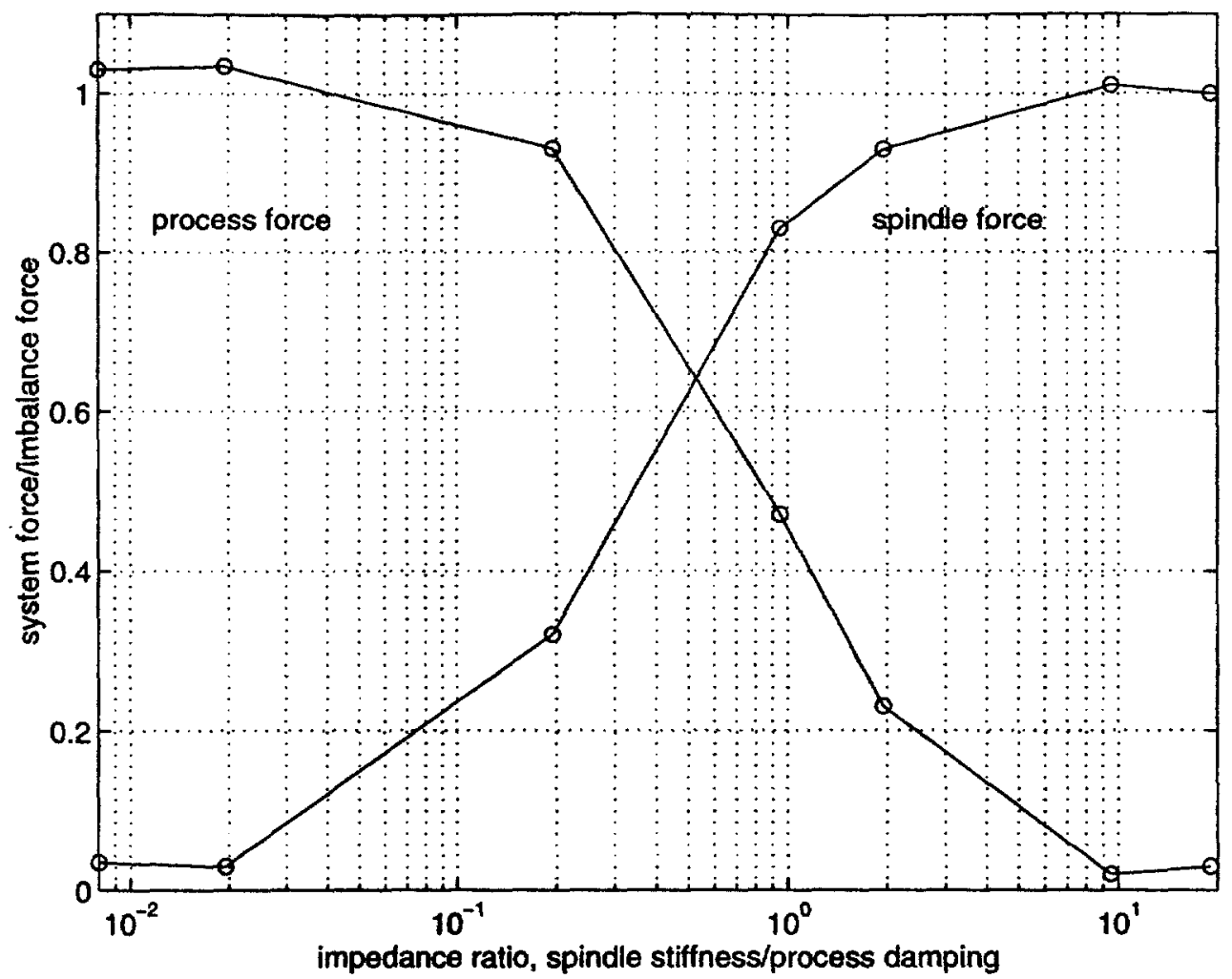

Figure 5-2: Effect of varying spindle stiffness on grinding process force and force transmitted to spindle.

from http://www.mathworks.com/.)

\subsubsection{Simulation Results}

For the purposes of the presentation at ASPE, this model was used to determine the effect of varying different machine parameters on the cutting process force. A sample output of the model can be seen in figure 5-2.

Figure 5-2 shows the effect of spindle stiffness on the cutting process force: as the spindle gets stiffer, more of the oscillating imbalance force component is transmitted to the machine through the spindle mount and less through the process. The abscissa is the ratio of impedances of spindle stiffness and damping in the cutting process. The ordinate is the cutting process force and grinding spindle force, normalized to the imbalance force. At some impedance ratio (approximately 0.5), the cutting process force and grinding spindle force are equal. The values in the graph were obtained by increasing the value for spindle 
stiffness in the model and keeping all other process parameters the same. The grinding speed in the model is $5000 \mathrm{rpm}$, which is unintentionally close to the modeled resonance of the workpiece rotor on its air bearing. Because of this resonance, the ratio of cutting process force to imbalance force is greater than 1 on the left edge of the graph. In the absence of resonances, this value would be expected to be equal to one with a soft spindle, meaning that 100 percent of the imbalance force was being transmitted through the process and none through the spindle bearing.

The model was also used at ASPE to show the effects of increasing process damping. (Recall that process damping can be increased by increasing the cutting area $A$ or decreasing the relative surface speed $V$.) As process damping is increased relative to spindle stiffness, the amount of spindle excursion decreases. This makes sense: with more damping in the cutting process the spindle shaft can be expected to vibrate less. See figure 5-3; here, spindle excursion was normalized to the excursion of a free-running spindle. Note in figure 5-3 that on the far right, the normalized spindle excursion is asymptotic to a flat line. This is because, at a high level of process damping, the system is overdamped and no further reduction in spindle excursion can be derived from additional damping. The value of the asymptote, in this case 0.78 , is dependent on grinding spindle stiffness. The stiffness of the grinding spindle sets the free-running excursion of the spindle given an input force imbalance (by Hooke's Law, $F=K x$ ). The dependent variable, normalized spindle excursion, is obtained by taking the ratio of in-process excursion to free-running excursion. Since free-running excursion is dependent almost entirely on spindle stiffness, it is spindle stiffness which sets the value for the high-end asymptote. A higher value for spindle stiffness results in a higher value of the asymptote. This makes sense physically: in very stiff systems one would expect very little change in excursion with change in process damping, and thus the asymptote would be close to 1 .

Also the transfer function between input imbalance force and cutting process force over a range of cutting speeds was found for this model. This is presented in figure 5-4. This transfer function was computed by varying the grinding speed while keeping the other parameters the same in the simulations. The resonant peak at approximately $22,000 \mathrm{rpm}$ is the resonance of the tool spindle on its air bearing, based on the parameters chosen in this model. The plot has the shape of a typical second-order system transfer function: it looks like a spring (nearly flat response) below resonance and a free mass above resonance 


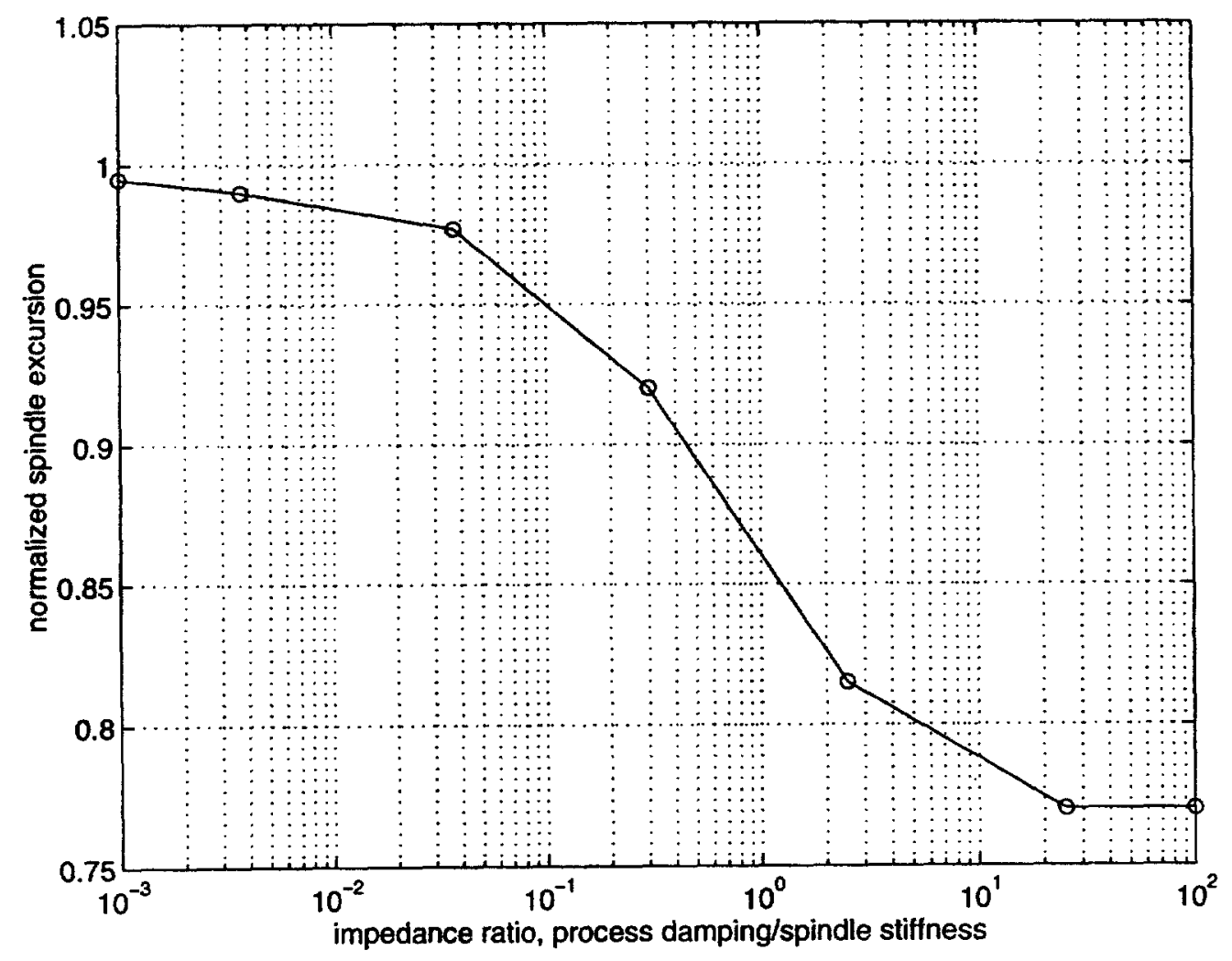

Figure 5-3: Effect of increasing process damping on spindle excursion. 


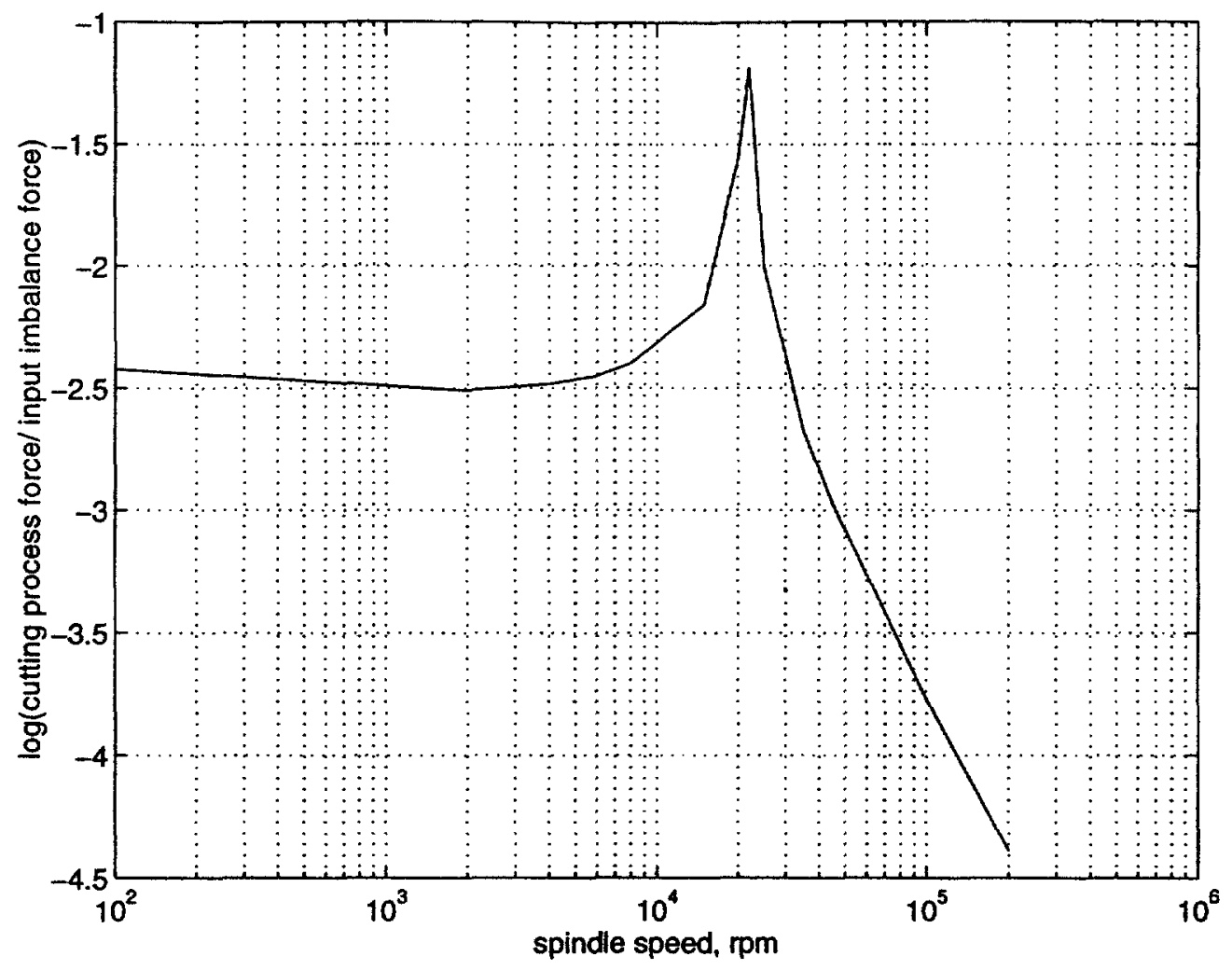

Figure 5-4: Magnitude transfer function between imbalance driving force and process force for ASPE model.

(dropping with a slope of $-20 \mathrm{~dB} /$ decade). This graph shows that in this model, imbalance oscillation is attenuated with a relatively steep slope above the resonance of the grinding spindle on its bearing. From this set of simulations, there is little influence from the resonance of the workpiece spindle on its bearing, which in this case occurs at 2,300 rpm. It should also be noted that the ratio between cutting process force and imbalance force is small over all frequencies; this is because in this simulation the value for damping given by $\frac{A}{K p V}$ is small compared to the stiffness of the spring between the grinding rotor and the machine. Most of the imbalance force is carried in this spring. With a relatively "soft" cutting process model such as the one used in these simulations, the dominant predictor of the imbalance-cutting process transfer function is the frequency response of the grinding spindle on its bearing.

These graphs are provided as an example of the potential usefulness of this modeling technique. It is relatively easy to derive a lumped-parameter model for a large machine 
Table 5.1: Modes of JR3-Westwind system used in analytical model

\begin{tabular}{|c|c|l|}
\hline \hline Frequency, $\mathrm{Hz}$ & Rot. Speed, rpm & Mode shape \\
\hline 96.65 & 5,800 & rigid-body rotation about load cell Y \\
113.25 & 6,800 & rigid-body rotation about load cell X \\
128.76 & 7,720 & rotation about load cell X \\
163.29 & 9,800 & rigid-body rotation about load cell $\mathrm{Z}$ \\
289.51 & 17,370 & rigid-body rotation about top corner of spindle \\
323.19 & 19,400 & rigid-body rotation about spindle tail \\
464.29 & 27,850 & rigid-body rotation about spindle shaft \\
\hline
\end{tabular}

tool; a model such as this highlights the complexity of machine dynamics. Such a model could prove useful in machine design as well as tolerancing for imbalance or truing.

\subsection{Explicit Analytical Model}

This section discusses an analytical model derived from the mode shapes and frequencies determined with STAR modal analysis. The procedure in obtaining this model was to isolate desired modes to be included, determine independent equations of motion for each mode based on the mode shape, and then drive those equations of motion numerically with an imbalance forcing function. Then predicted error motion of the metrology ball could be determined by combining the results of the simulation in the relevant sensitive directions. To begin, I decided on the JR3 (undamped) system, and looked at the frequencies and mode shapes given in table 5.1 .

Looking at the animation results, I decided to throw out the mode occurring at $128 \mathrm{~Hz}$. The relative displacements of the nodes in the model were large for adjacent points of the stiff spindle structure, which suggested that the mode was small in amplitude compared to the others. (The others were significantly "cleaner" rigid-body motions.) Also, looking at the frequency spectra from the accelerometers, the mode at $128 \mathrm{~Hz}$ was small in amplitude compared to the others.

Discounting the mode at $128 \mathrm{~Hz}$, I was left with six modes. The first three were rigidbody rotations approximately about the center of the load cell. The fourth, fifth and sixth modes had centers of rotation about other points on the structure. Since I possessed quoted values from the load cell manufacturer for stiffness of the load cell, I could derive equations 
Table 5.2: Moments about load cell axes

\begin{tabular}{|c|c|}
\hline Moment & Value, $\mathrm{kg} \mathrm{m}^{2}$ \\
\hline$I_{x}$ & 10.41 \\
$I_{y}$ & 7.584 \\
$I_{z}$ & 1.140 \\
\hline
\end{tabular}

of motion for each of the first three modes. For each mode, I had the natural frequency and rotary spring stiffness. From these I could determine the rotary moment of inertia. For example, in the case of the moment-about- $\mathrm{X}$ direction, the natural frequency of the mode is given by

$$
\sqrt{\frac{K_{M x}}{I_{x}}}=2 \pi f_{M x}
$$

where $K_{M x}$ is the load-cell stiffness in the moment-about-X direction, $I_{x}$ is the (unknown) moment of inertia about that axis, and $f_{M x}$ is the natural frequency of that mode, in this case $113.25 \mathrm{~Hz}$. By solving the equation for $I_{x}$ I obtained a value for the rotary moment of inertia of the spindle about load cell X. Using this procedure I was able to estimate rotary inertia about each of the three load cell axes. These can be seen in table 5.2.

At this point $I$ had stiffness and rotary moment of inertia for three fundamental modes, enough to formulate three analytic equations of motion describing those modes. The other three modes presented more of a problem: I did not have the convenience of supplied stiffness data for those modes. For each, I only had natural frequency and mode shape, which was a rotation about a point on the spindle (not the load cell center, as before). However, it is possible to determine a translated rotary moment of inertia about any arbitrary center of rotation using the parallel-axis theorem. I already had rotary moments of inertia about each of the three load cell axes, so moments of inertia about any arbitrary point relative to the structure could be computed. For these "arbitrary" points I chose the stationary points (centers of rotation) of the remaining three modes. Using the computed moment of inertia about the center of rotation of the mode and the natural frequency of that rotation, it is straightforward to compute an equivalent stiffness value using an equation of the form of equation 5.2. By this procedure I was able to formulate approximate equations of motion for the other three modes. 
Table 5.3: Moments and equivalent stiffnesses for six modes

\begin{tabular}{|c|c|c|}
\hline axis & $\mathrm{I}, \mathrm{kg} \mathrm{m}^{2}$ & $\mathrm{~K}, \mathrm{~N} \mathrm{~m} / \mathrm{rad}$ \\
\hline load cell $\mathrm{x}$ & 10.41 & $3.84 \mathrm{e} 6$ \\
load cell $\mathrm{y}$ & 7.584 & $3.84 \mathrm{e} 6$ \\
load cell $\mathrm{z}$ & 1.140 & $1.20 \mathrm{e} 6$ \\
top corner spindle & 9.580 & $3.17 \mathrm{e} 7$ \\
spindle tail & 12.41 & $5.12 \mathrm{e} 7$ \\
spindle centerline & 6.159 & $5.24 \mathrm{e} 7$ \\
\hline
\end{tabular}

I first had to find moments of inertia about the stationary points of modes four, five, and six. Each calculation required two applications of the parallel-axis theorem: the first to translate from load-cell center (where the moments in table 5.2 are defined) to approximate spindle center, and the second to translate from there to the stationary point on the spindle (the axis of rotation of the structure for the mode in question). This was necessary because the parallel-axis theorem must always translate between the center of mass of a body and any other point in space. For the approximate center of mass of the spindle, I chose a point that was geometrically in the center of the large mass.

The parallel axis theorem in this case is:

$$
I_{t r a n s}=I_{c t r}+m d_{p}^{2}
$$

where $I_{\text {trans }}$ is the translated moment of inertia, $I_{c t r}$ is the moment of inertia about the center of mass, $m$ is the mass of the body, and $d_{p}$ is the distance between the parallel axes. I obtained the mass of the spindle, $m$, from the manufacturer. By the applying the parallel-axis theorem twice for each of the remaining three modes, I was able to compute moments of inertia for those modes. From there, an application of an equation like 5.2 for each mode gives an equivalent stiffness for that mode. Table 5.3 presents the rotary moments of inertia and equivalent stiffness about each of the centers of rotation for the six modes.

Knowing the stiffness and moment of inertia of each mode allows analytic expression of the unforced equation of motion for that mode, for some arbitrary choice of damping constant. (It would be potentially more accurate to use damping values provided by the modal analysis software; this was not done in this case due to time constraints.) The general 
form for the rotational equations of motion for the unforced system are given by:

$$
I \ddot{\theta}+B \dot{\theta}+K \theta=0
$$

where $I$ is the rotary moment of inertia, $\theta$ is the angle of rotation, $B$ is the damping, and $K$ is the stiffness. Each equation of motion is forced by its own forcing function, which in this case is a moment function specified by the imbalance driving the system and the distance from that force input to the center of rotation of the relevant mode. Adding in forcing, we have

$$
I \ddot{\theta}+B \dot{\theta}+K \theta=A d \sin (\omega t+\phi)
$$

where $A$ is the amplitude of imbalance force, $d$ is the moment distance from that force to the center of rotation of the mode, $\omega$ is the spindle speed (frequency of imbalance driver), and $\phi$ is the phase lag between a reference phase and the phase of maximum excitation of the relevant mode. In this system, the $+Y$ direction serves as the reference direction for phase. Since the modes involve rotation in different directions, each is excited in some phase relative to the others by the rotating imbalance sinusoid.

For the equations of motion, I arbitrarily chose $B$ so that $\zeta=0.1$; all modes are lightly damped for solution stability. ( $B$ is related to $\zeta$ by $B=2 \zeta \sqrt{K I}$.) Earlier incarnations of the algorithm without any damping had not been numerically stable.

After deriving the six independent equations of motion for the system, including damping and excitation phase, I made the decision to drop the highest mode, for two reasons. The principal reason is that the mode is about the spindle centerline and therefore it is hard to excite with a rotating imbalance. Second, the high frequency of the mode places it beyond the range of my experiments and other testing (about 28,000 rpm). For these reasons I decided to drop the mode from consideration.

At this point I had five equations of motion that, when solved, would yield angles of rotation about each of five points on the structure. It remained to translate these rotations to displacements of the metrology ball. In this way the model could be compared to empirical data from the capacitance gauges. The translation from rotation to ball translation for each mode was accomplished using small-angle approximations (valid because the angles of rotation are on the order of thousandths of a radian!) and trigonometry. Each rotational 
mode contributes to translation in two of the three sensitive directions of measurement. By adding the contributions from each mode, the overall spindle error motion in each of the three directions can be predicted.

The end result of this derivation is a model that predicts spindle deflections due to imbalance at any rotational speed, given any imbalance mass. This model uses knowledge of the system mode shapes obtained through empirical modal analysis and supplied values for system stiffness. This modeling technique is powerful because it is rooted in experimental modal data but permits analytical insight into the dynamics of the system. The model is flexible in that the relevant parameters are accessible: it is easy to analytically change values of system damping, for example. It is also easy to see how the model works, and the user has great flexibility in choosing a solution method for the differential equations of motion. This can be contrasted with the forced response synthesis model (see the next section) available in some modal analysis packages, which is essentially a "black box" algorithm which outputs system motion based on driving-point sinusoids and mode shape data.

Appendix B gives the MATLAB ode code and post-processing code (translation from rotation angle to metrology ball translation) for the analytical model used in this thesis. The next chapter will present results of the analytical model for the JR3 (no damping) system alongside experimental spindle error results.

\subsection{Implicit STAR Model}

To predict motions of the spindle structure driven by imbalance, I also used the Forced Response Synthesis feature which is built in to the STAR modal analysis software. This feature allows the user to drive the system at a specified node or nodes with a sinusoidal force oscillation. Following the calculation of one structural response, the user may superimpose a second result. In this way, many sinusoidal drivers may excite the system at different nodes, with different phases. After the simulation is completed, the user is provided with a table of results for each node. These results consist of amplitude of oscillation and phase relative to the driving function. In addition, the user can look at an animated display of system motion due to the driving forces. Refer to [17] for more information.

For the Westwind spindle-load cell system I simulated imbalance by the superposition of two sinusoids, exciting the structure at the location of the imbalance ring. One sinusoid, at 
90 degrees to the other in direction, acted with a 90-degree phase lag. This pair of sinusoids looks like a rotating imbalance to the structure model in STAR. The force amplitude of the sinusoids was chosen to equal the theoretical imbalance magnitude at each rotational speed. In this way, spindle motion due to imbalance was predicted. Since it was quick to run compared to deriving an analytical model, I ran this simulation for each of three systems: JR3-Westwind, JR3-Westwind-damping, and Kistler-Westwind. The results are presented alongside the experimental results in the next chapter.

This method has the advantage over the analytical method of requiring practically zero time: once the modal data is processed, the forced response can be calculated immediately. As mentioned in the last section, the disadvantages are that there is less insight into system dynamics with this method and there is no explicit system model in which to vary parameters. 


\section{Chapter 6}

\section{Experimental and Model Results}

This chapter presents and discusses results from both the experimental capacitance gauge and load cell data collected and two different models: the analytical lumped-parameter model and the output from STAR software forced response synthesis.

\subsection{Sample Experimental and Model Outputs}

Figure 6-1 shows a sample output from the data acquisition system. The upper plot shows the counter pulse output: this is the once per revolution signal that indicates when the timing window has passed the photo-eye. Looking at this signal allows determination of the revolution speed of the spindle. It also serves as a reference for determining phase angle information from the other signals. In the case of the JR3 data acquisition, which required two CPUs and three A/D boards, one counter pulse signal was measured by each board to serve as a reference between the three boards. In this manner differences in clock speed, for example, could be determined.

The lower plot of figure 6-1 shows a sample capacitance gauge output, synchronous with the counter signal. This is for the radial1, or Y-direction capacitance gauge, which at this speed is predominantly showing the effects of a ball decenter of 27.5 microinches. (Making for a peak-to-peak displacement of 55 microinches.) Before each data measurement, the amount of ball decenter and phase relative to the timing window was measured by hand. These values were later subtracted out using Tehranchi's identity [18] for summing complex exponentials, to determine the amplitude and phase due to imbalance alone. (See Appendix $\mathrm{C}$ for further discussion.) For each test, three synchronous measurements from 

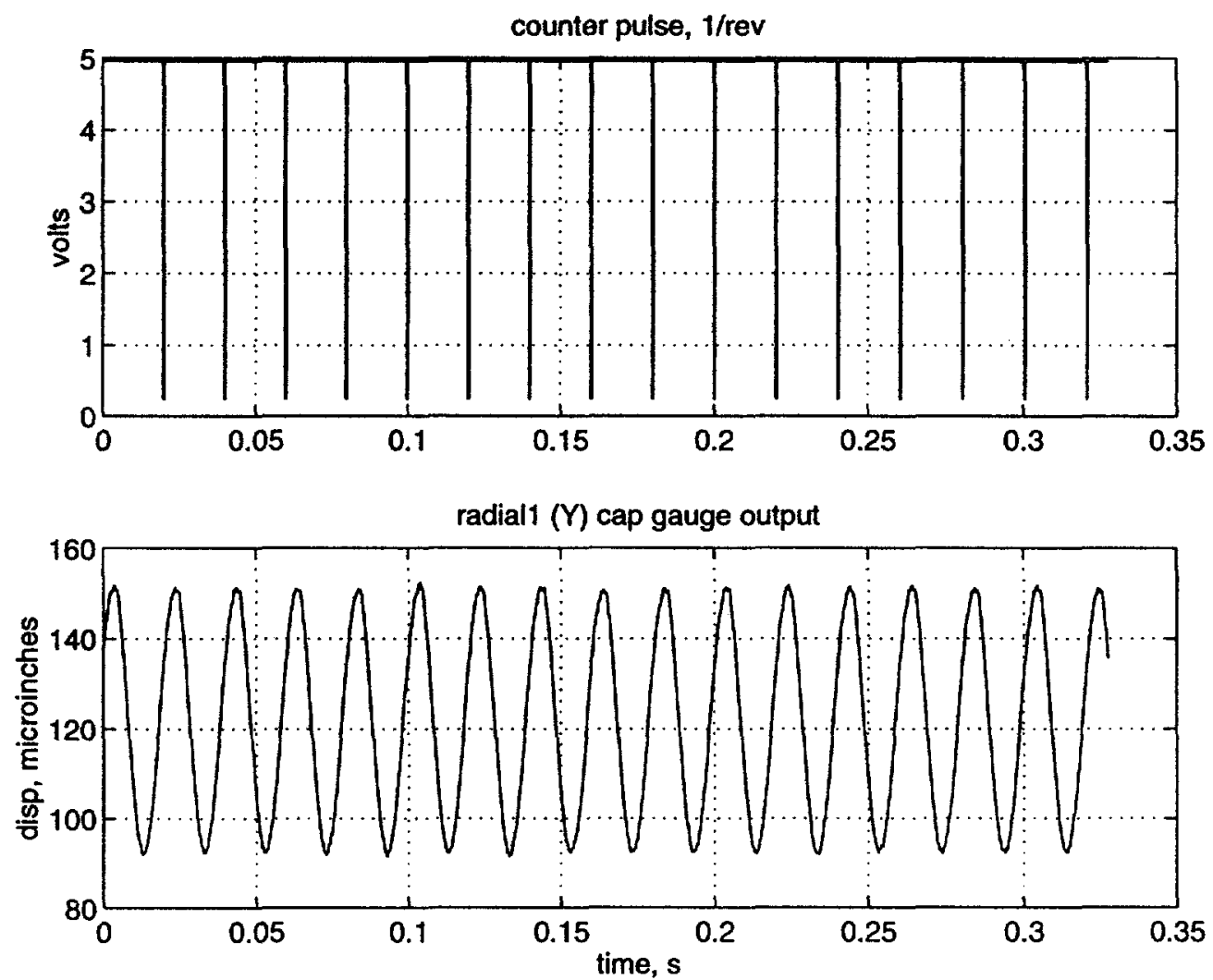

Figure 6-1: Sample data trace from experiment, $3000 \mathrm{rpm}$ with undamped JR3 system. 

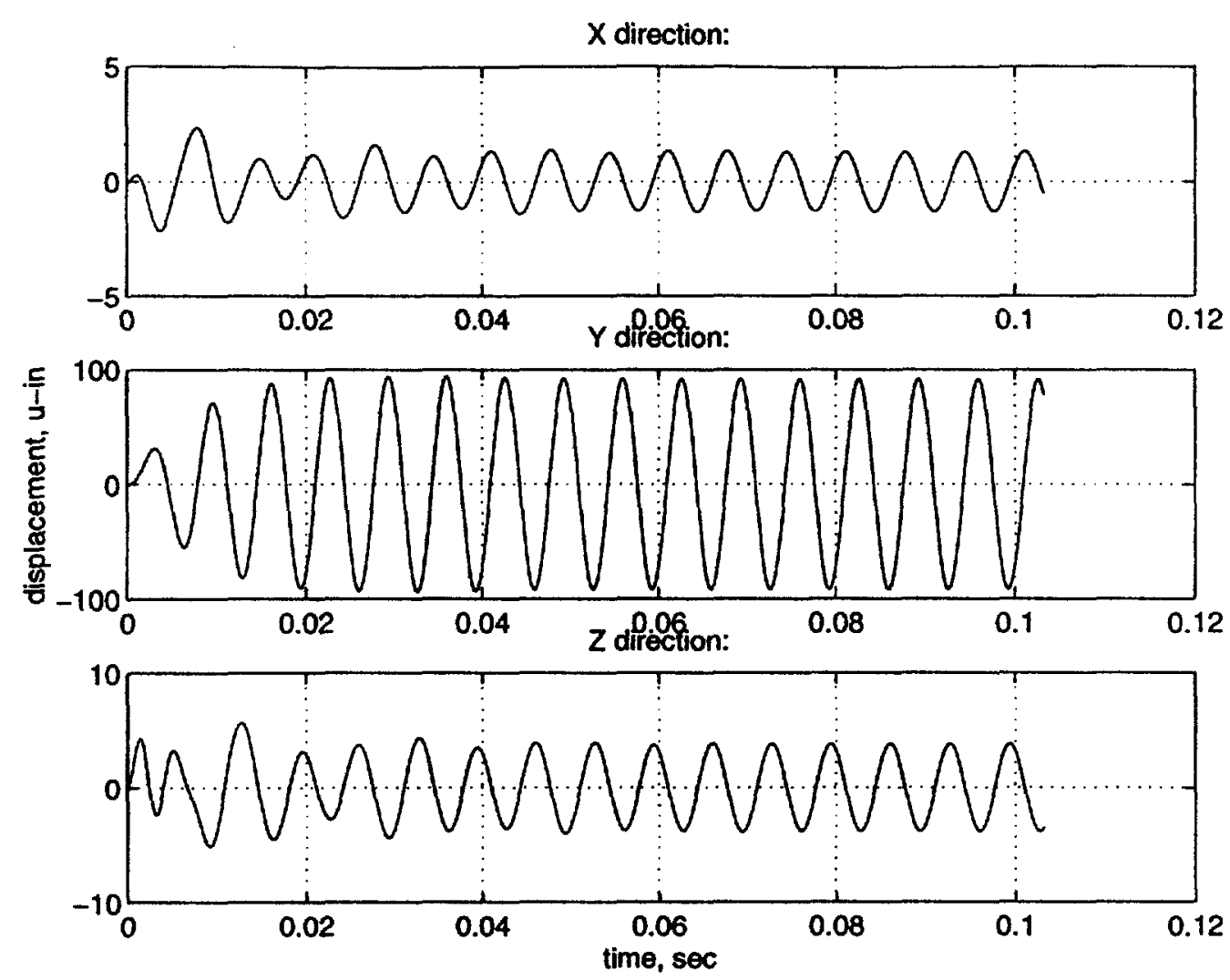

Figure 6-2: Sample output trace from analytical model, $9000 \mathrm{rpm}$ with undamped JR3 system. Displacements are in microinches.

the capacitance gauges were made, and in the case of the JR3 load cell, six force directions were also measured synchronously (three for the Kistler load cell). This data was taken over $1 / 3$ second at a sampling rate of $25 \mathrm{kHz}$ per channel. (The combined data file size for each JR3 test is over 800 kilobytes.) Amplitude and phase information for each signal of interest was determined graphically, using MATLAB routines.

A sample output from the analytical lumped-parameter model can be seen in figure 6-2. This figure shows the model running with an excitation speed of $9,000 \mathrm{rpm}$ and mass " $A$ " for imbalance. The three graphs show the translational motion in the $\mathrm{X}, \mathrm{Y}$, and $\mathrm{Z}$ directions, respectively. In the first 0.03 seconds of simulation, the initial conditions are settling out (due to the light damping in this model; earlier versions with no damping showed many frequency components for each trace!) and the model is settling into a stable once-perrevolution oscillation which can be compared with the experimental data gathered from the capacitance gauges. Phase and magnitude information from the model were determined 
graphically using MATLAB scripts.

\subsection{Undamped JR3 System: Empirical Error Motion and Two Models}

This section compares capacitance gauge data for the JR3 system with no damping material and the two models discussed previously. Those models are the analytical model, which is presented in the middle graph, and the STAR forced response synthesis, which is presented in the bottom graph. There are six figures: three for oscillation amplitude in each direction and three for phase (relative to the driving force) in each direction. Figures 6-3, 6-5, and 67 compare the amplitude information while figures 6-4, 6-6, and 6-8 compare the phase information.

Looking at figure 6-3, which looks at X-direction or axial data, we notice first that there is a large discrepancy in the oscillation magnitudes predicted by the two models and the capacitance-gauge data. In this case, take as an example the resonance at $5,800 \mathrm{rpm}$. (This mode is pictured in figure 4-2.) Taking the analytical model first, the discrepancy could be due to any of several different factors. For one, the damping of oscillatory modes in the model was chosen more or less arbitrarily. Since it is damping that entirely determines peak height at resonance, the choice of $\zeta$ as 0.1 may be much larger than the actual system damping, and may be pushing down the resonant peaks.

Another factor in the discrepancy between experiment and the analytical model may be unmodelled dynamics: the analytical model assumes direct excitation of the spindle structure (stator) at the imbalance ring location. In actuality, the imbalance is acting on the spindle shaft which is coupled to the stator through an air bearing. For the purposes of this model, the air bearing stiffness was assumed to be "infinite" (perfectly rigid coupling between shaft and stator). The stiffness of the air bearing was assumed to be much larger than the stiffness of the load cell. In actuality, however, the apparent shaft stiffness at the shaft's end as quoted by the manufacturer is indeed finite. $\left(4.5 \times 10^{7}\right.$ Newtons per meter at $10,000 \mathrm{rpm}$; air bearing stiffness is a function of rotational speed.) When compared in a moment sense with the rotational load cell stiffnesses which dominate.the rigid-body modes of this system, the values are similar, suggesting that air bearing stiffness is an effect which should have been included in the model. See Appendix D for details on the comparison of 

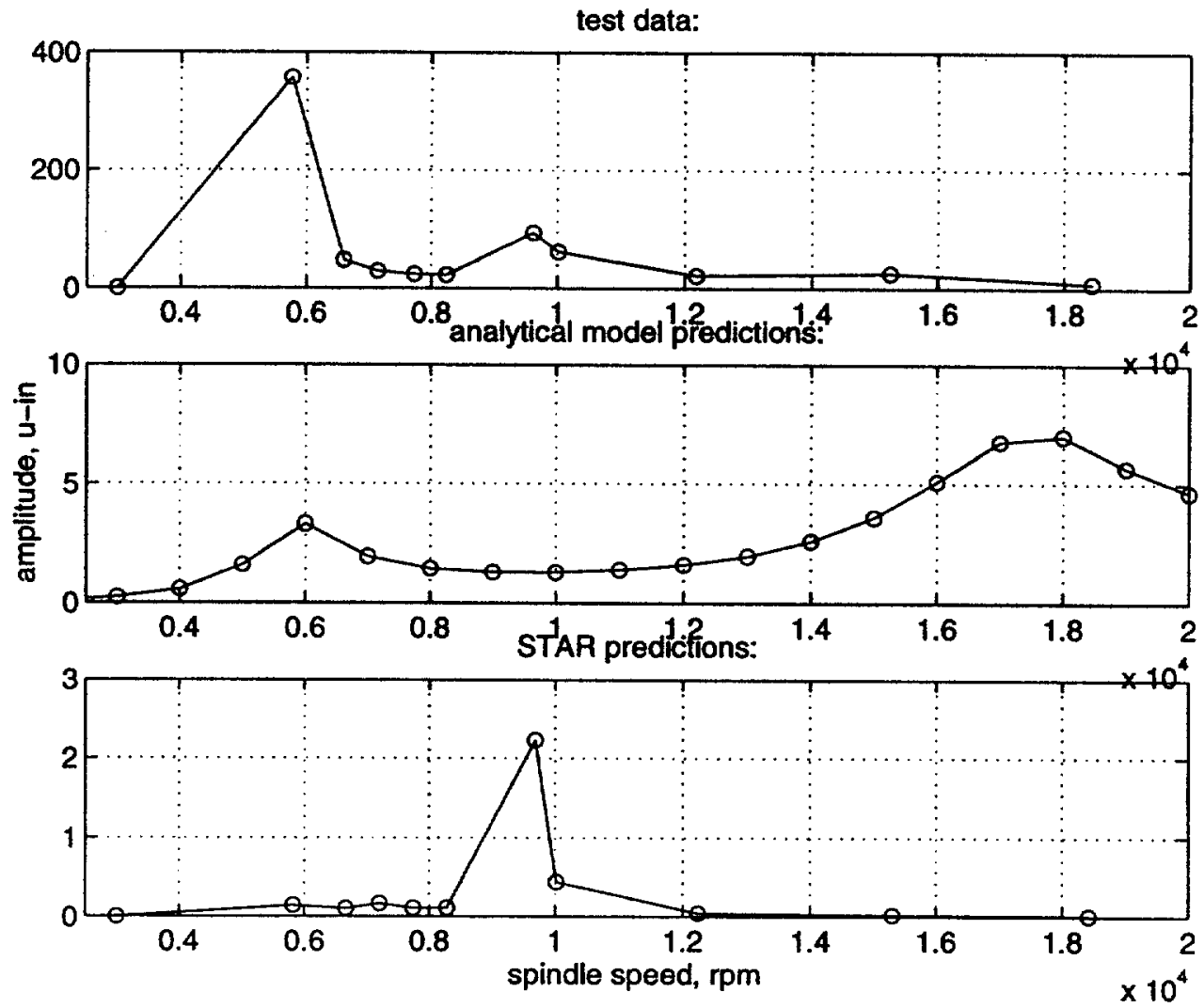

Figure 6-3: JR3 system without damping material, as sensed in the $\mathrm{X}$ (axial) direction; comparison of data and 2 models for displacement amplitude. 
these stiffnesses and a discussion on the unmodelled dynamics of the air bearing shaft.

The analytical model missed the 9,700 rpm peak, but this is understandable given the simplified mode shapes used by the analytical model: at this frequency the analytical model has an equation of motion which specifies pure rotation about the load cell Z-axis only. For this reason the model does not predict any appreciable motion of the ball in the $\mathrm{X}$-direction. In reality, the system is predominantly rotating about the load cell Z-axis but it is also exhibiting some smaller motions in other directions, including axially. Obviously, in the real system these effects are apparent. One problem with constructing a model "backed out" from mode shape data is that it in simplifying the mode shapes to construct analytical equations of motion, some information is lost. This, too, is a deficiency of this model. The STAR model nicely predicts this motion at $9,700 \mathrm{rpm}$.

The analytical model predicts a high-speed resonance band at 16,000 to $18,000 \mathrm{rpm}$ that did not show up in the capacitance-gauge data or the predictions of STAR. This band appears analytically because the model was constructed with high-end resonances at 17,400 rpm and 19,400 rpm. It is not clear why this band does not appear in the capacitance-gauge data or the STAR simulation. One possible explanation is that damping in the analytical model, which was arbitrarily chosen, was incorrect. Another is that the finer frequency mesh of the analytical model (see figure 6-3) shows resonances that the empirical capacitancegauge data and STAR prediction miss. However, I would like to make the point here that it was not my goal to tweak the analytical model until it matched the experiment perfectly and then claim perfect understanding of the system; rather, I wanted to demonstrate that modeling machine dynamics in this way is of potential use.

In the case of the STAR model (bottom graph of figure 6-3), the discrepancy between oscillation amplitudes could be caused by incompatibility between the source of the modal spectra and STAR. The STAR software determines mode shapes from input spectra from another package - in this case a Hewlett-Packard spectrum analyzer. The compatibility of the engineering units used for the spectra between these two pieces of software was set to be the same but it is difficult to verify whether they are in fact the same. In many cases the predictions of the STAR package are off by a more or less constant ratio from the actual amplitudes.

However, I do not have a good explanation for the failure of the STAR package to predict the large resonant peak in the capacitance-gauge data at 5,800 rpm. Across the three JR3 
directions (X, Y, and Z), the STAR system failed to predict any appreciable motion below 9,700 rpm. A disadvantage of using the STAR forced response synthesis is that it is a "black box" in the software and its results are hard to check. The failure to predict the mode could arise from many mechanisms, including software error. One possible explanation, as with the analytical model, is failure to take into account relevant machine dynamics. The STAR model was excited through a node on the rotor, but when the modal data was taken the stiffness of the rotor was different than at operating speed. Also, the system in the modal tests was not excited via the rotor, but on the stator. So, the knowledge of the motion of the rotor is limited in STAR to one accelerometer on the end of the rotor. This does not give a wealth of information on rotor-stator dynamics. But I reiterate that I do not have a satisfactory explanation as to why STAR is not predicting large-amplitude oscillation at $5,800 \mathrm{rpm}$.

Phase angle predictions and data in general show much poorer agreement than magnitude data. By phase angle, we mean the lead or lag of oscillation in a sensitive direction relative to the force input oscillation in that direction. The discrepancy is potentially due to a number of reasons: our experimental phase data is measured graphically from the data files and thus there is some room for error in the measurement. Additionally, there is overlap in phases: many different values of phase angle are equivalent. For example, it is nearly impossible to tell the difference between a 175-degree lead in phase and a 175-degree lag, yet when graphed the two look wildly different. In practice they are only 10 degrees apart in terms of their effects on the system. Figure 6-4 shows the measured and predicted phase for the undamped JR3 system in the $\mathrm{X}$ (axial) direction. There is very little agreement between the data and the model predictions for phase angle. Phase information is mainly presented here for completeness.

Figure 6-5, which looks at magnitude of oscillation in the $Y$, or radial1 direction, shows nice agreement between the capacitance-gauge data and the model data, with the exception of a resonance at 6,700 rpm that was missed by both the analytical model and STAR. Again, both of these models had knowledge of a mode at this frequency: this mode corresponds to a rocking motion about the $\mathrm{X}$-axis of the load cell (pictured in figure 4-3). It is unclear why neither model predicted a resonant peak at 6,700 rpm. As stated before, possible explanations include unmodelled dynamics of the bearing shaft and errors in damping value. In the case of STAR it is difficult to pinpoint the source of errors. I would like to state 


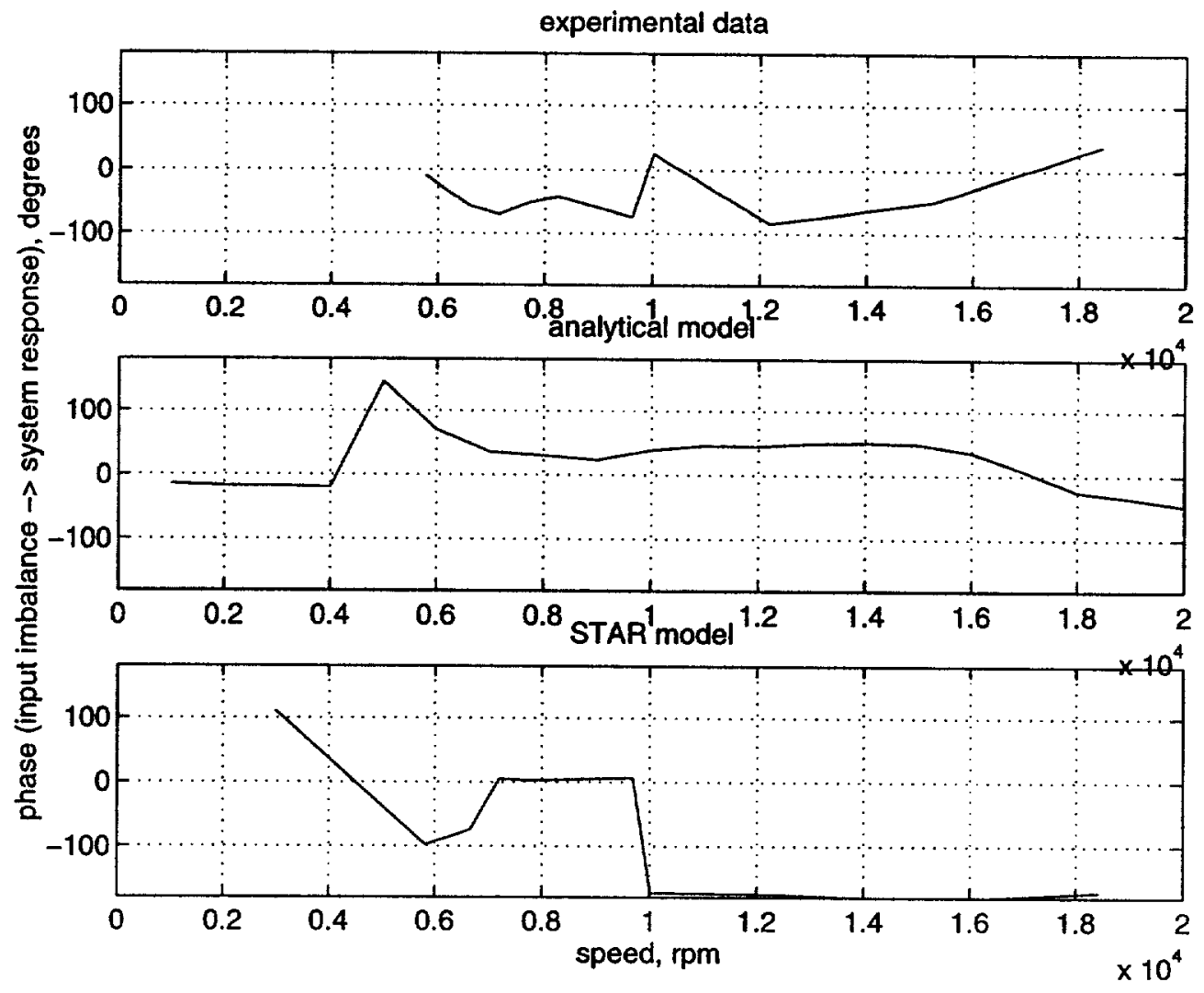

Figure 6-4: JR3 system without damping material, as sensed in the $\mathrm{X}$ (axial) direction; comparison of data and 2 models for phase relative to excitation input. 

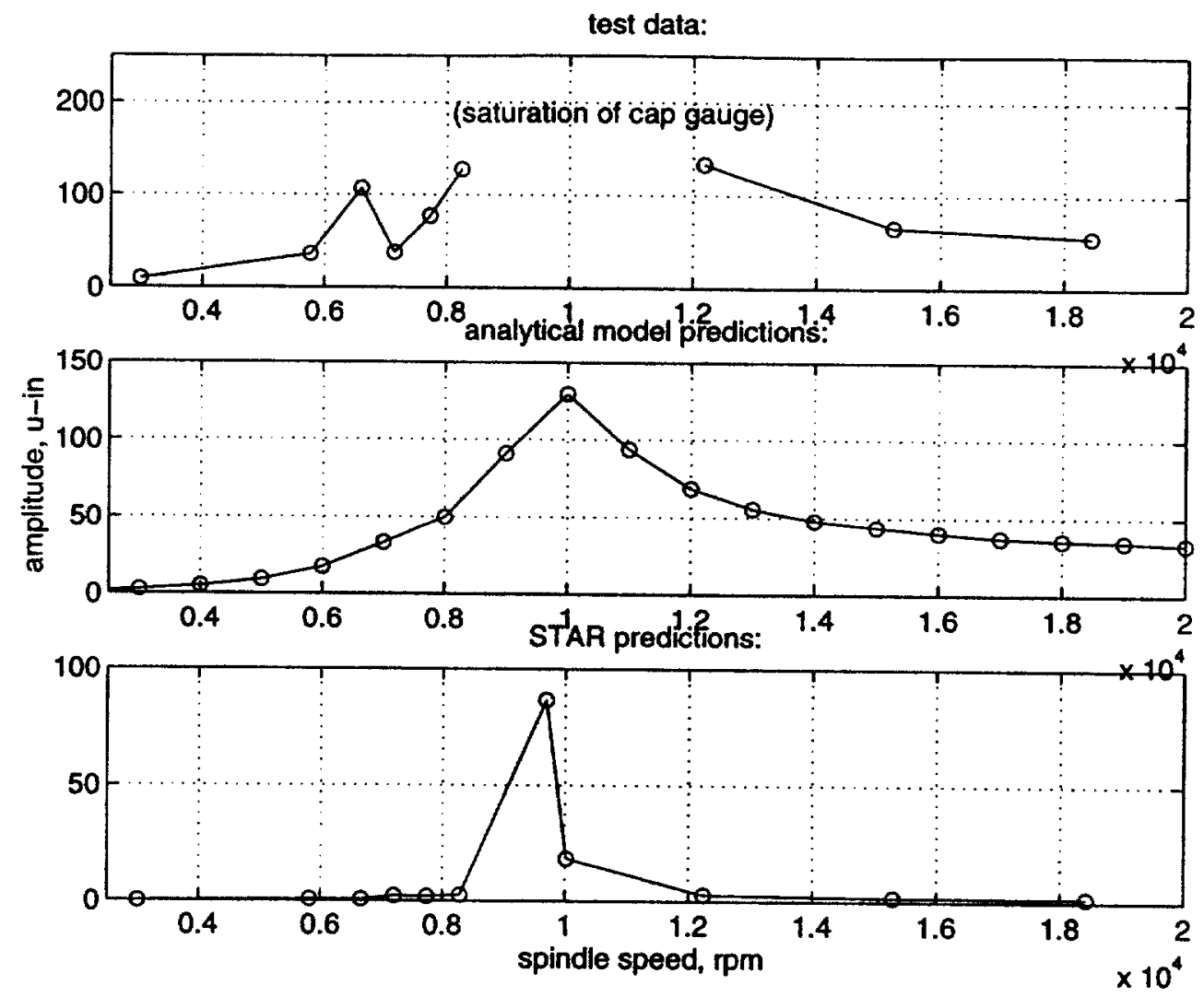

Figure 6-5: JR3 system without damping material, as sensed in the Y (radial1) direction; comparison of data and 2 models for displacement amplitude.

again that I do not have a satisfactory explanation for this discrepancy.

The large resonance at around 10,000 rpm was predicted well by both models. In the experimental data, the capacitance gauge, which has a range of 1000 microinches, was saturated over this frequency range! The undamped JR3 system shows a lot of oscillation in this range, which corresponds to the entire spindle rotating about the Z-axis of the load cell. (This mode is pictured in figure 4-4.)

There is better agreement in the phase data for the Y-direction than the X-direction. (See figure 6-6.) Both models predict phase starting at zero degrees and ending up at -180 degrees ( +180 is equivalent), with a transition through approximately $10,000 \mathrm{rpm}$. The STAR phase prediction, having fewer data points, shows a very abrupt transition to -180 degrees. The capacitance-gauge data (top plot) shifts in phase at around 7,000 rpm, to about +50 degrees, which is equivalent to a shift downward to -135 degrees. It then continues to lag as frequency increases, like the STAR prediction. 


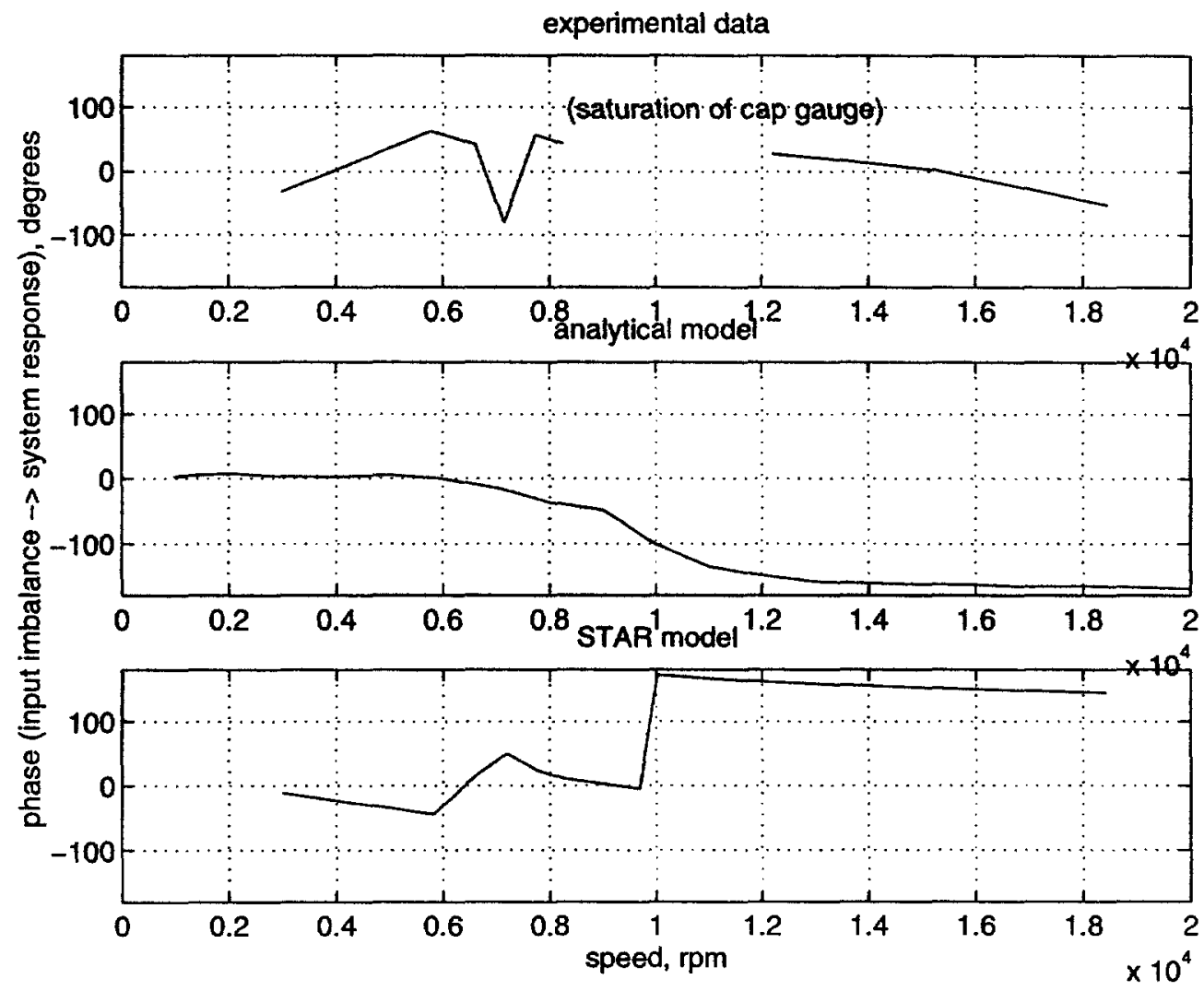

Figure 6-6: JR3 system without damping material, as sensed in the Y (radial1) direction; comparison of data and 2 models for phase relative to excitation input. 
test data:
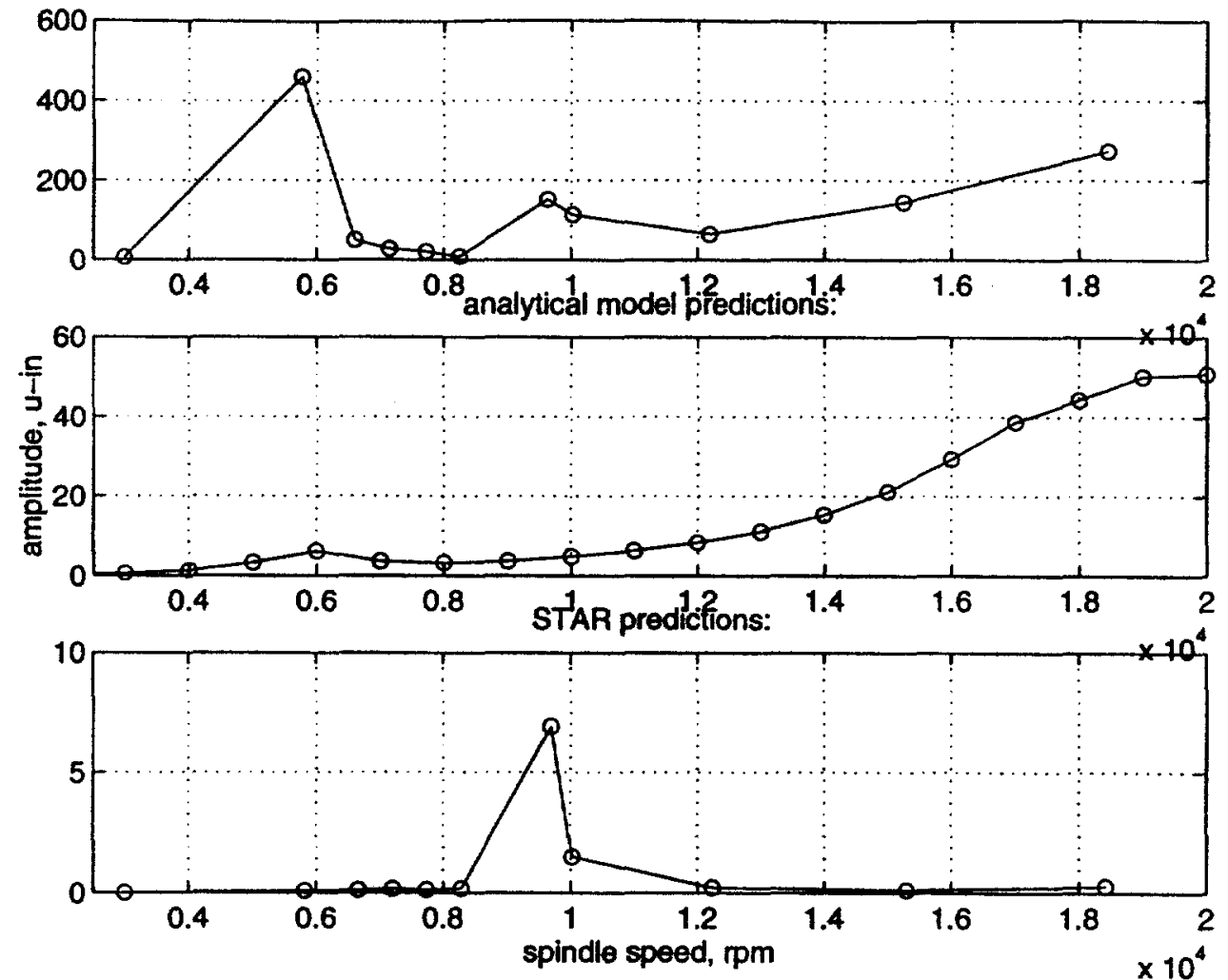

Figure 6-7: JR3 system without damping material, as sensed in the Z (-radial2) direction; comparison of data and 2 models for displacement amplitude.

Figure 6-7 shows the capacitance-gauge amplitude of oscillation data versus two models in the Z-direction (negative radial2 direction). Like the $\mathrm{X}$-direction(figure 6-3) there is some discrepancy in the locations of natural frequencies in this direction. The STAR model missed a significant low-frequency oscillation at 5,800 rpm (this is the same resonance as discussed earlier, in the discussion of figure 6-3). Again, it is unclear what was the cause of STAR's failure to predict the resonance.

The analytical model missed the 9,700 rpm peak, but this is again understandable given the simplified modes used by the analytical model: at this frequency the analytical model has an equation of motion which specifies pure rotation about the load cell about the Z-axis. Therefore, this simplified model does not predict any Z-axis translation of the spindle end. In reality, however, this effect is observable. Again, this is a deficiency of the simplified analytical model.

It is difficult to find much agreement in the phase information for the $\mathrm{Z}$ (negative radial2) 


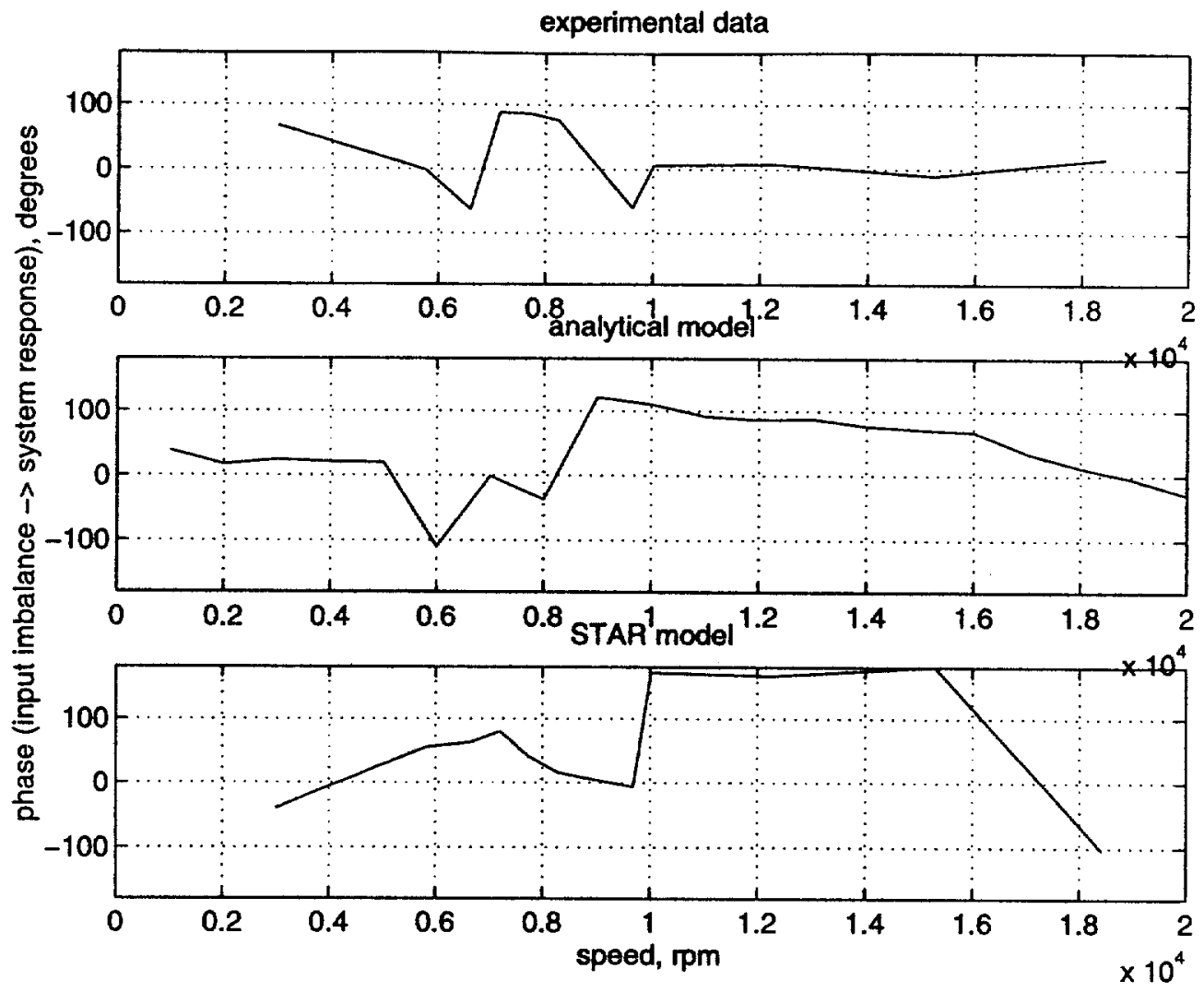

Figure 6-8: JR3 system without damping material, as sensed in the Z (-radial2) direction; comparison of data and 2 models for phase relative to excitation input.

direction. This data is plotted in figure 6-8.

It should be mentioned here that while the softness of the spring and variety of modes encountered with the JR3-Westwind setup provided for an interesting set of experiments, it is not recommended that this system be used for doing any in-process measurements of grinding forces. Such a soft load cell has the disadvantage of introducing a very compliant member into the machine structure and by this mechanism potentially introducing large error motions into the grinding process.

\subsection{Kistler System: Empirical Error Motion and STAR Predictions}

This section compares the results of the Kistler capacitance-gauge data to the predictions made by STAR forced response synthesis for the frequency range from 3,000 to $20,000 \mathrm{rpm}$. 

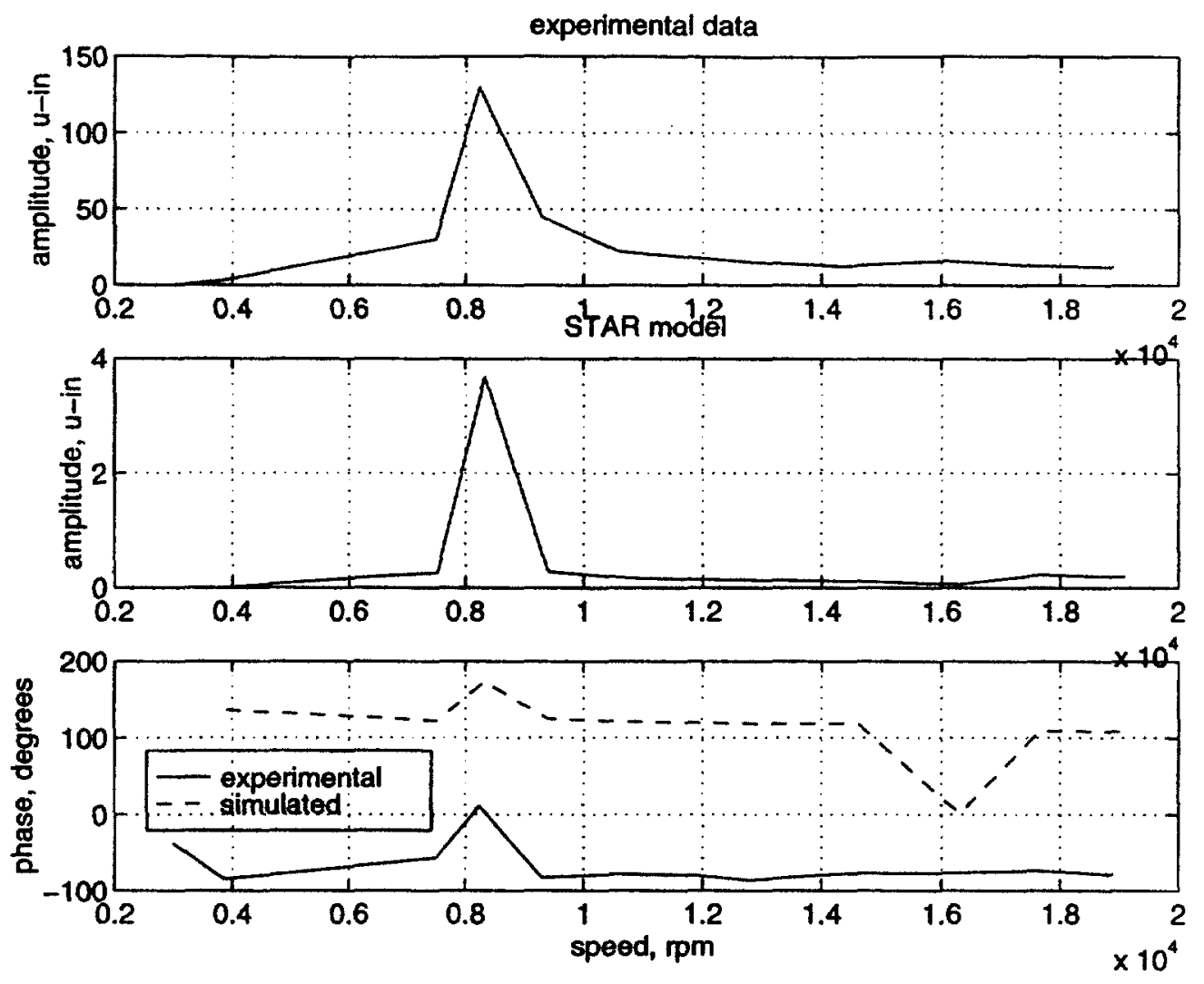

Figure 6-9: Kistler system, as sensed in the $\mathrm{X}$ (axial) direction; comparison of data and STAR model.

No analytical model was developed for this system.

In general, there is very good agreement between capacitance-gauge data and the STAR predictions for the stiffer Kistler system. The exception is again the magnitudes of oscillation predicted by the STAR routine: they are much smaller in magnitude than the test results. This effect suggests that there is a systematic scaling error in our data, possibly arising from incompatibility of units between the frequency spectra from each accelerometer and the STAR program which combines those spectra into mode shapes.

Figure 6-9 shows the response of the Kistler system to imbalance in the X, or axial, direction. There is good agreement in the placement of a natural frequency at around 8,300 rpm. The stiffer Kistler system exhibits a much cleaner frequency response spectrum than does the JR3, in general. There are fewer natural frequencies to avoid in operation of the machine. The phase angle has roughly the same shape between the test data and STAR prediction but does not agree numerically. 

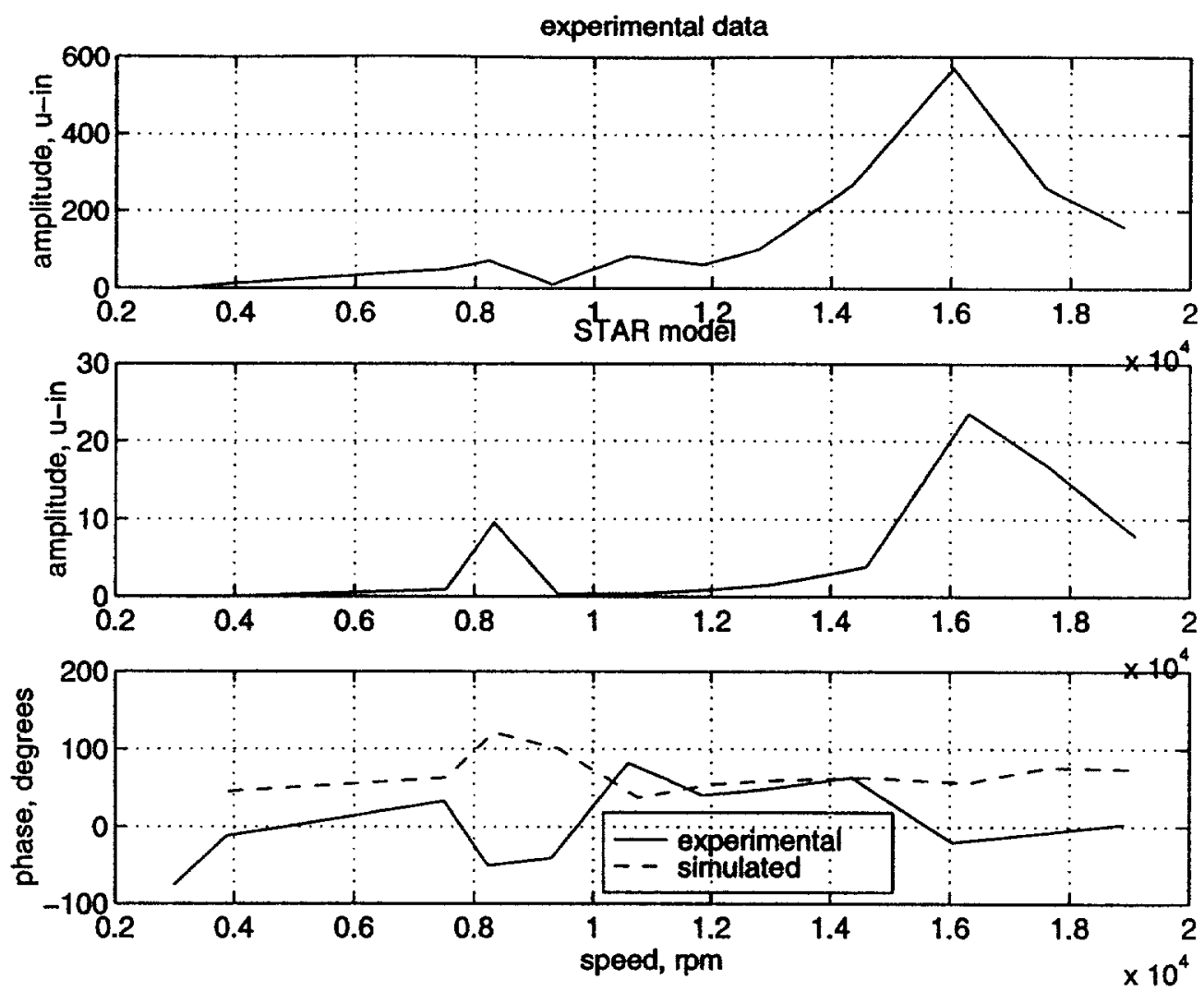

Figure 6-10: Kistler system, as sensed in the $\mathrm{Y}$ (radial1) direction; comparison of data and STAR model.

In figure 6-10, we see the amplitude and phase response of the Kistler system in the $\mathrm{Y}$, or radiall, direction. Again, there is good agreement between the predictions of the STAR model and test data, although STAR predicts a relatively larger oscillation at $8,300 \mathrm{rpm}$ than in actuality. The phase angle data is also in agreement over much of the speed range of the experiments.

Lastly, figure 6-11 shows the Z-direction response of the Kistler system over the frequency range tested. There is again good agreement between the STAR model and capacitance-gage data with regards to placement and relative magnitude of resonant peaks in this sensitive direction. The shape of the phase curves show some agreement, as well.

The Kistler data makes a strong case for using modal analysis to predict potential ranges of large spindle error. With this stiffer structure there is even better agreement between the predictions of the STAR modal package and test data. Should this Westwind-Kistler system be used as part of a larger machine tool, the results of this modal testing would be 

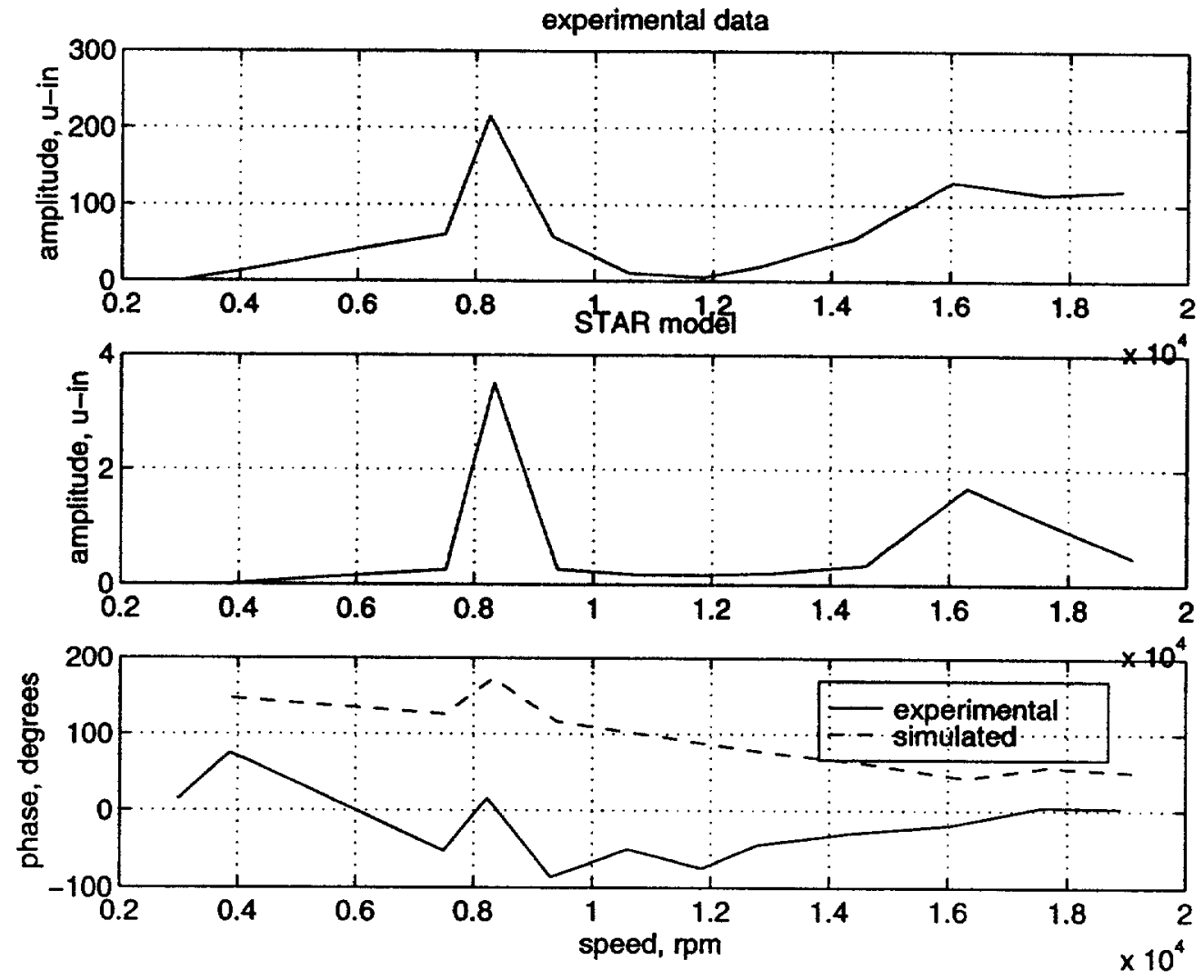

Figure 6-11: Kistler system, as sensed in the Z (-radial2) direction; comparison of data and STAR model. 
valuable in selecting a grinding speed for this system, or in specifying the demands that such a spindle places on the dynamics of the machine.

\subsection{Discussion of Error Motion Results}

The important result from the last two sections is that modal testing is potentially very beneficial in precision grinding applications. To fully instrument a spindle to obtain spindle error motion is a time-intensive process. It requires setting up capacitance gauges to measure spindle error motion in every relevant direction. It requires a data acquisition system for the capacitance gauges, which is not commercially available for high-speed applications such as this one. It requires a great deal of experimental testing to determine stable operation frequencies for the machine. And at the end, the machine or process designer only has error motion quantified in the directions in which they tested, and very little or no understanding of the fundamental causes underlying that error motion.

On the other hand, modal testing is an established field with many software packages for sale and an abundance of technical help available. A modal test of a machine tool and data reduction could be performed by an experienced modal analysis expert in much less time than a full spindle metrology test, and the modal testing software and equipment is much more readily available than the custom hardware and software currently required for spindle metrology. Once the modal test is completed, the machine tool mesh can be computer-animated to determine relative displacements due to excitation forces: be they imbalance error, seismic error, or other arbitrary force inputs. This sort of test can give the machine or process designer an intuitive understanding of the causes behind spindle error motion. Looking at a frequency spectrum of the modal data (excited at the spindle rotor) will tell the process designer which speeds to avoid in operation of the machine (resonances), in each sensitive direction. The modal data can also be incorporated into generating analytical models (such as the one presented here) which may give the process designer more understanding of the mechanisms at work. Finally, packages like STAR give the user the option of adding lumped-parameter elements to their mesh, to investigate the effects of adding damping to the system, for example. (Although this was not investigated for this thesis.) The cost advantages of modal analysis over conventional spindle error motion testing, at least for a first cut in understanding machine dynamics, are clear. 
However, I would also like to express some reservations about modal analysis as a panacea for spindle error determination: it should not be used without thought and understanding to accompany it. Some results of this modal analysis forced response synthesis are troubling in that they do not predict error motion which is observed in the real system. In the case of the JR3-Westwind system, STAR forced response synthesis did not predict spindle error motion at all below $9,700 \mathrm{rpm}$, when that error motion was in fact present in the experiments. (At higher frequencies there was decent agreement between STAR predictions and experiment.) The source of this discrepancy is not understood and further work would be needed to determine the cause of this discrepancy. In balance, modal analysis is a promising alternative or supplement to traditional spindle error motion metrology.

This preliminary work also suggests that it will be worthwhile to pursue further work on developing analytical models from modal test data. It would be helpful to start with a much simpler system than the spindle-load cell of this thesis and perform empirical modal analysis while deriving an analytical model alongside that analysis. In this way the analytical model would be well-understood and rooted in physical reality. If, for example, modal analysis was performed on a one-degree-of-freedom mass-spring system, the simple analytical model derived alongside the modal data could be compared easily with the modal data. For example, it would be easy to select a value for damping in the analytical model based on modal testing results. In this way, the two processes would be well understood with at a fundamental level. This kind of understanding would lead to better intuition on more complicated models.

Overall, I would recommend the judicious use of commercially-available modal analysis packages as an alternative to conventional spindle metrology methods. The insight and time advantages of modal analysis make it worthwhile. Using analytical modeling to predict error motion may also be useful, although I would recommend modeling the system from the "ground up", instead of the approach taken here, which was to back out the analytical model parameters from STAR modal testing results. This approach is of some use but fails to adequately capture all of the relevant system dynamics. 


\subsection{JR3 System: Effects of Adding Damping Material}

This section looks at the effect of adding damping material to the JR3-Westwind system on spindle error motion. Both capacitance gauge data and STAR predictions for error motion with damping will be presented.

Figure 6-12 shows a comparison of axial, or X-direction, amplitude for the JR3 system. It is clear from this picture that adding the Sorbothane damping material significantly reduces error motion due to imbalance at spindle speeds near resonance. At 5,800 rpm, the undamped system exhibits more than 350 microinches of axial excursion amplitude. Adding the damping material reduces that error motion to around 80 microinches, less than a quarter the amplitude of the original. At the next resonant peak, 9,700 rpm, the addition of Sorbothane cuts the oscillation amplitude roughly in half. It can be seen from the figure that at frequencies away from the resonant peaks, the damping material has much less effect.

The lower half of figure 6-12 shows STAR simulation results for the same two systems. Although (as mentioned before) STAR failed to predict the large resonant peak at 5,800 $\mathrm{rpm}$, it did in general predict the same sort of behavior from the damped system. That is, at frequencies of resonance the peak amplitude is reduced significantly, while at frequencies away from those resonances there is little change from the undamped system.

Subjectively, during the data acquisition for the tests with the damping material, the effects could be noticed immediately. Without the addition of Sorbothane, the spindle "buzzed" near the resonances when excited with imbalance. The oscillations were large enough that by simply touching the spindle in different locations, one could predict the magnitude of the oscillatory mode shape at that frequency. It was also audible to the operator that the spindle was near a resonance. Once the damping material was added, however, the system quieted down noticeably around the resonances. The physical shaking had also significantly lessened.

In figure 6-13, which shows the effect of adding damping material in the Y-direction, we can see the same sort of results as in the axial direction. Here during the experimental testing, the capacitance gauge signal in this "radial1" direction was saturated at 9,700 and $10,000 \mathrm{rpm}$ without the damping material added - meaning more than 0.001 " of radial amplitude at these excitation speeds. Once the damping was added, however, this dropped 

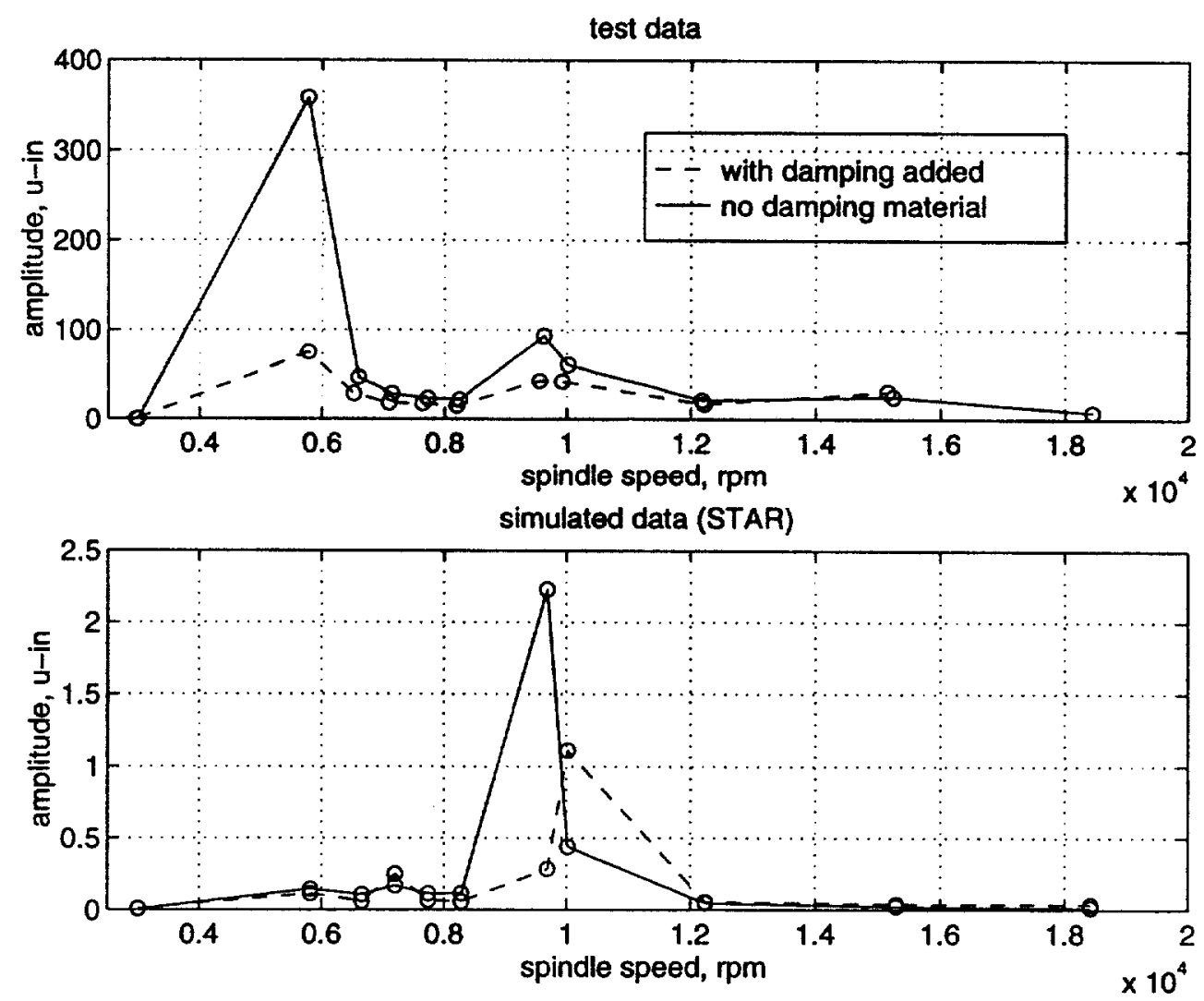

Figure 6-12: JR3 system with and without damping material, as sensed in the X (axial) direction; capacitance-gauge and STAR simulation results for displacement amplitude. 

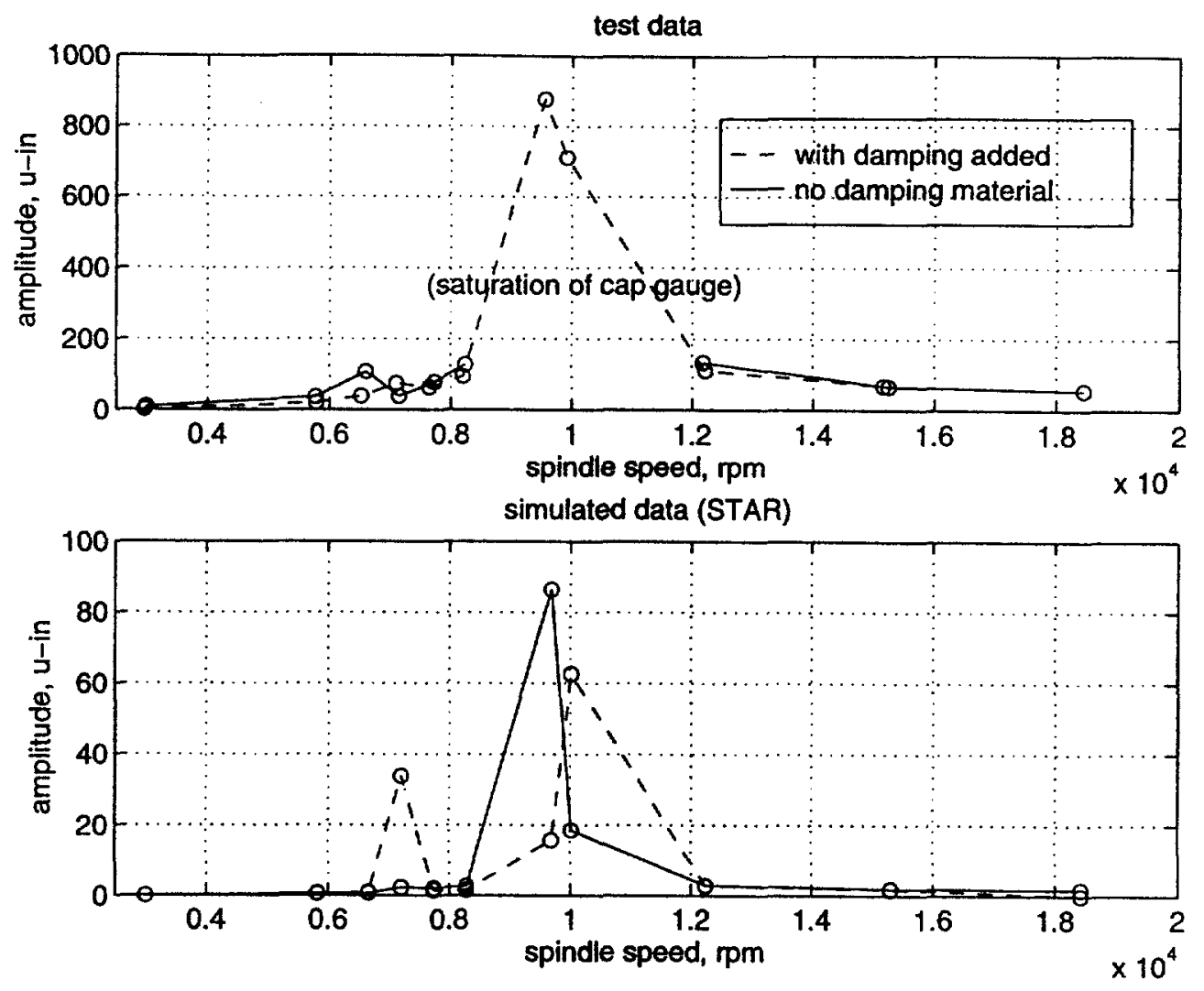

Figure 6-13: JR3 system with and without damping material, as sensed in the $\mathrm{Y}$ (radial1) direction; capacitance-gauge and STAR simulation results for displacement amplitude.

to around 900 microinches. In this case, by damping out the oscillation in a momentabout-Z mode, the damping material was acting in a purely shear fashion (less effective for Sorbothane than compression). This is because the damping material lay in a plane defined by the $\mathrm{X}$ and $\mathrm{Y}$ axes. It is postulated that with compression damping material placed more effectively (i.e. in the $\mathrm{XZ}$ or YZ-plane) for this disruptive mode, the results would be even better. In addition, different types of damping material are available which are designed for shear damping applications; in a machine design using a setup such as this (lots of rotational modes), shear damping may be more effective.

The slight damping of this mode by the addition of the damping material can be seen even more clearly in the STAR graph (lower half of figure 6-13). STAR predicts roughly a $30 \%$ reduction in amplitude at this frequency. This can be compared to a $50 \%$ reduction in the X-direction (see lower half of figure 6-12).

Similar results hold for the system as viewed in the Z-direction (figure 6-14). The greater 

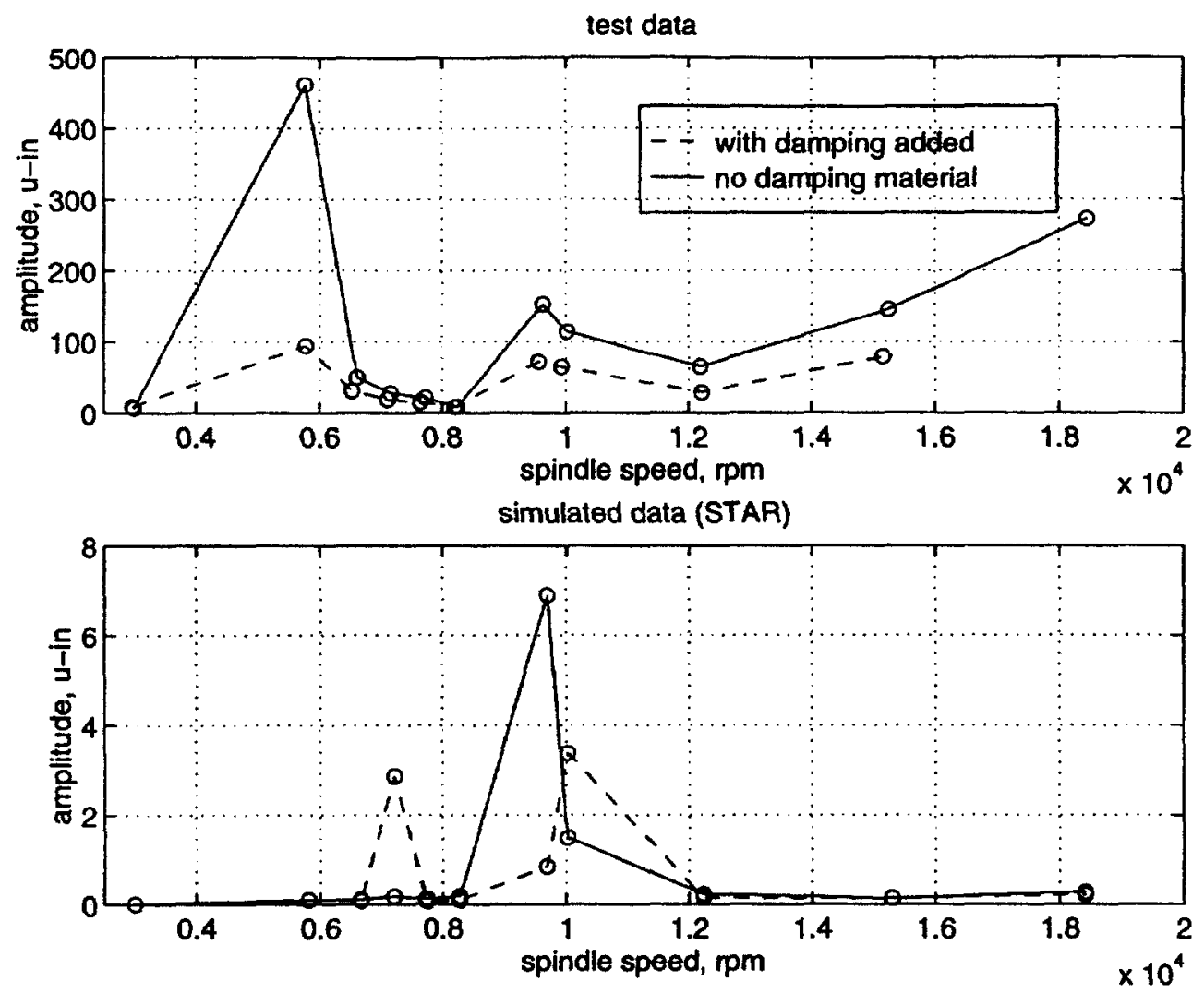

Figure 6-14: JR3 system with and without damping material, as sensed in the Z (-radial2) direction; capacitance-gauge and STAR simulation results for displacement amplitude.

the oscillation amplitude, the more the effect of damping on the system. The addition of damping material serves to level off the frequency response curve of the system. It is useful, then, to add damping to a grinding system for rejecting imbalance disturbance, especially if the natural frequencies of the grinding system are not known. If modal analysis or some other method of determining natural frequencies in the system has been performed, then in most cases it is easy to avoid those frequencies in the selection of spindle speed. If, however, it is impossible to avoid running the system near resonance, or if the resonances are not well understood, the addition of damping to the system may be the best first cut at reducing the effects of imbalance or other process error sources. 


\subsection{Force Transmitted to Machine Structure}

In addition to causing error motion in the spindle, an unbalanced spindle also drives the surrounding machine structure with an oscillating force. Figure 6-15 shows a plot of the experimental force in the Y-direction over a range of spindle speeds. It is clear from this plot that the significantly stiffer Kistler load cell transmits higher forces to the surrounding machine than the softer JR3 setup. It should be noted that this plot is not normalized for input imbalance force, which is also shown as a reference. For the purposes of comparing forces driving the machine structure, I chose the Y-direction because it exhibited large displacements in the capacitance gauge measurements (due to moment-about- $Z$ oscillation), and it would be the chosen sensitive direction for peripheral grinding with this setup. The force transmitted in the JR3 system with damping was not considered as this force is not directly comparable with the others; the damping material transmits some force which is not measured.

The tradeoffs between the two load cells are clear. With the stiffer Kistler load cell, there is in general less spindle error motion due to imbalance but more force transmitted into the surrounding machine structure. A softer load cell (JR3) exhibits more oscillation amplitude but less force transmitted out from the imbalance source into the machine. These conclusions can be extended to design of precision grinding machines: designing a stiffer bearing or support structure will improve error motion seen by the part, but the downside is that potentially more force due to imbalance will excite the machine structure. If the surrounding structure has some "Achilles' heel" - a relatively compliant member in some frequency range - imbalance forces may play into error motion through the mechanism of indirect excitation of the structure, which would also carry through to part error. Again, ideally all relevant natural modes of the machine tool should be understood, to minimize potential errors on the part. With the understanding of machine mode shapes and frequencies would come the intelligent selection of process speed and placement of damping material, and avoid potentially expensive trial-and-error selection of grinding wheel speed and other process parameters. 


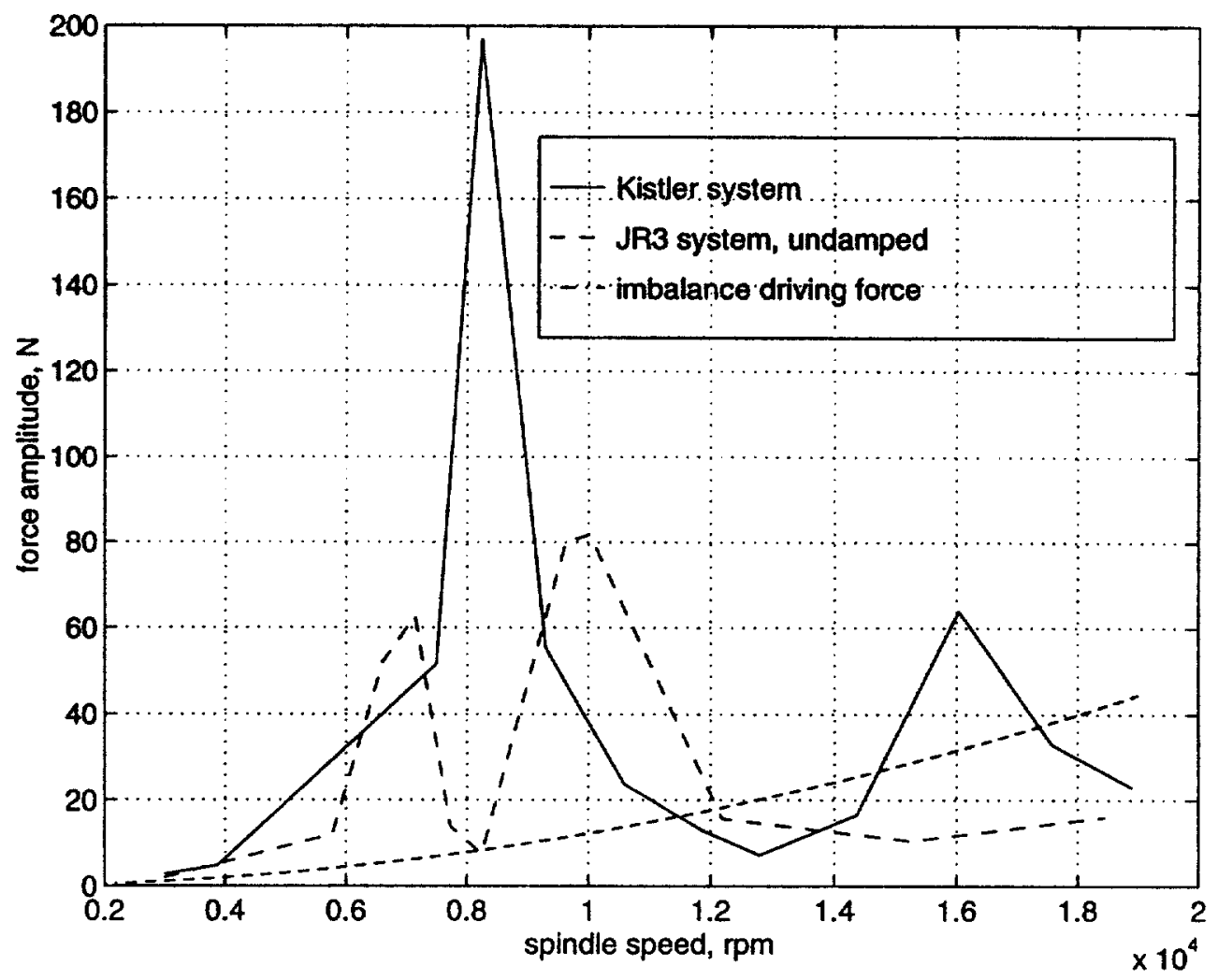

Figure 6-15: Comparison of force into machine structure, $\mathrm{Y}$ direction, JR3 and Kistler systems. 


\subsection{Compensating for Decenter with Imbalance}

One objective of this series of experiments was to determine whether, in practice, decenter of the grinding wheel can be compensated with imbalance. In this series of experiments, I used the Westwind spindle system with no load cell underneath (bolted directly to the large granite table). I did this to get around problems of resonance encountered in the load cell systems, and make for a "cleaner" test. I ran each test at 10,000 rpm. Before each run, I measured the location and amplitude of the decenter of the ball. I then rotated the imbalance ring to a specified phase angle ahead of the location of the maximum decenter. I did this for angles starting at zero degrees and advancing in increments of 45 degrees back around to zero again.

Figure 6-16 shows the results of this set of experiments. Along the abscissa one can see the advancing phase lead in degrees. The ordinate axis shows a "normalized decenter." By "normalized decenter," I mean the ratio of the output amplitude to the input decenter. This normalization was necessary because at the small levels of decenter involved in these tests (order of 30 microinches), some drift in decenter occurred between tests. (A 5 microinch drift between tests would change the decenter by $17 \%$.)

From the figure it is obvious that in conditions where the imbalance and decenter are coincident, the effect of an initial decenter is greatly magnified $-400 \%$ in these tests. As imbalance location physically advances relative to decenter, the magnification effect lessens. At one location only, 225 degrees, the initial offset was improved by the addition of imbalance. In this case an offset of 22.5 microinches was reduced to 11.3 microinches by adding an imbalance. This has implications for correcting decenter (truing error in grinding wheels) with imbalance. It is very difficult and time-intensive to true a grinding wheel. (This process can take on the order of a week in some precision grinding applications!) On the other hand, many grinding systems already come with in-process balancing (systems made by Balance Dynamics, for instance). If the grinding speed is chosen so that there are minimal adverse effects on the machine structure, it may be beneficial to apply a small amount of imbalance to the system to compensate for truing errors in the wheel. The dynamic balancers sold by Balance Dynamics work by sensing oscillation of the spindle stator (via an accelerometer) and moving high-density fluid (Halon) between chambers in a rotating assembly to minimize the shaking due to imbalance. If, instead, the balancer closed 


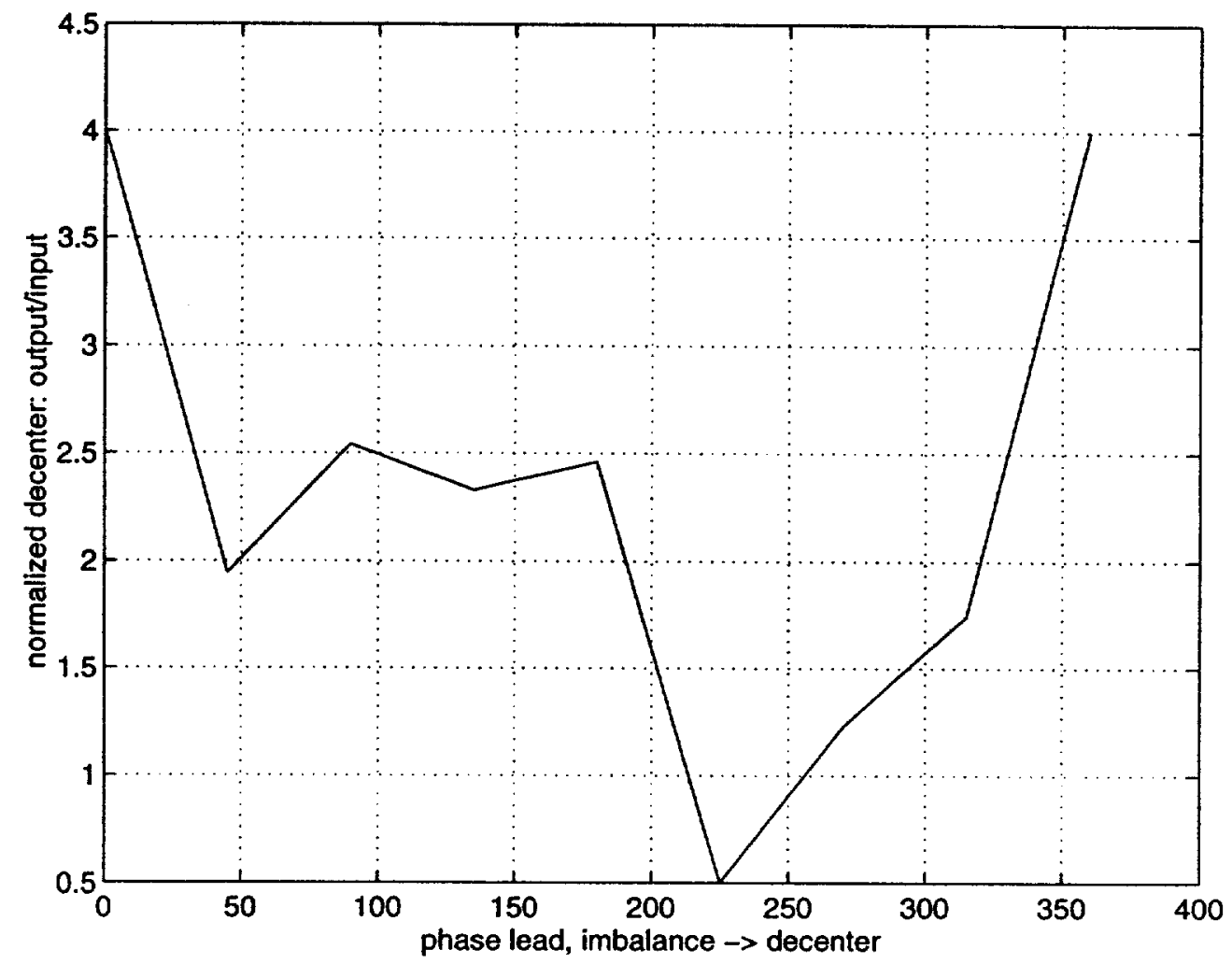

Figure 6-16: Compensating for decenter with imbalance: the effect of phase lead on normalized decenter. 
its loop around force sensed on the other side of the process (an in-process dynamometer, for example), once-per-revolution geometric errors in the wheel could be compensated for by applied imbalance; the closed-loop control system would take care of amplitude and phase of the compensating imbalance. This is clearly an area for potential future research.

\subsection{Effect of Increasing Mass of Imbalance}

Two series of experiments were performed to determine the effect of increasing imbalance mass on spindle error motion and force transmitted to the machine structure. Four set screws were used as imbalance masses, designated A, B, C, and D (refer to table 3.3). The spindle with undamped JR3 load cell was run at 3,000 rpm (below all resonances) and 5,800 rpm (approximately at the first mode). Figure 6-17 shows the increasing spindle error motion in the $\mathrm{Y}$-direction for both speeds, through the range of masses. Figure 6-18 shows the force transmitted to the machine at both speeds, through the range of masses. In both cases, the relationship between the dependent variable and the increasing mass is logarithmic-shaped. These graphs are provided as a reference to indicate what levels of spindle error motion and force transmitted to the machine structure can be expected as the amount of imbalance mass increases. 


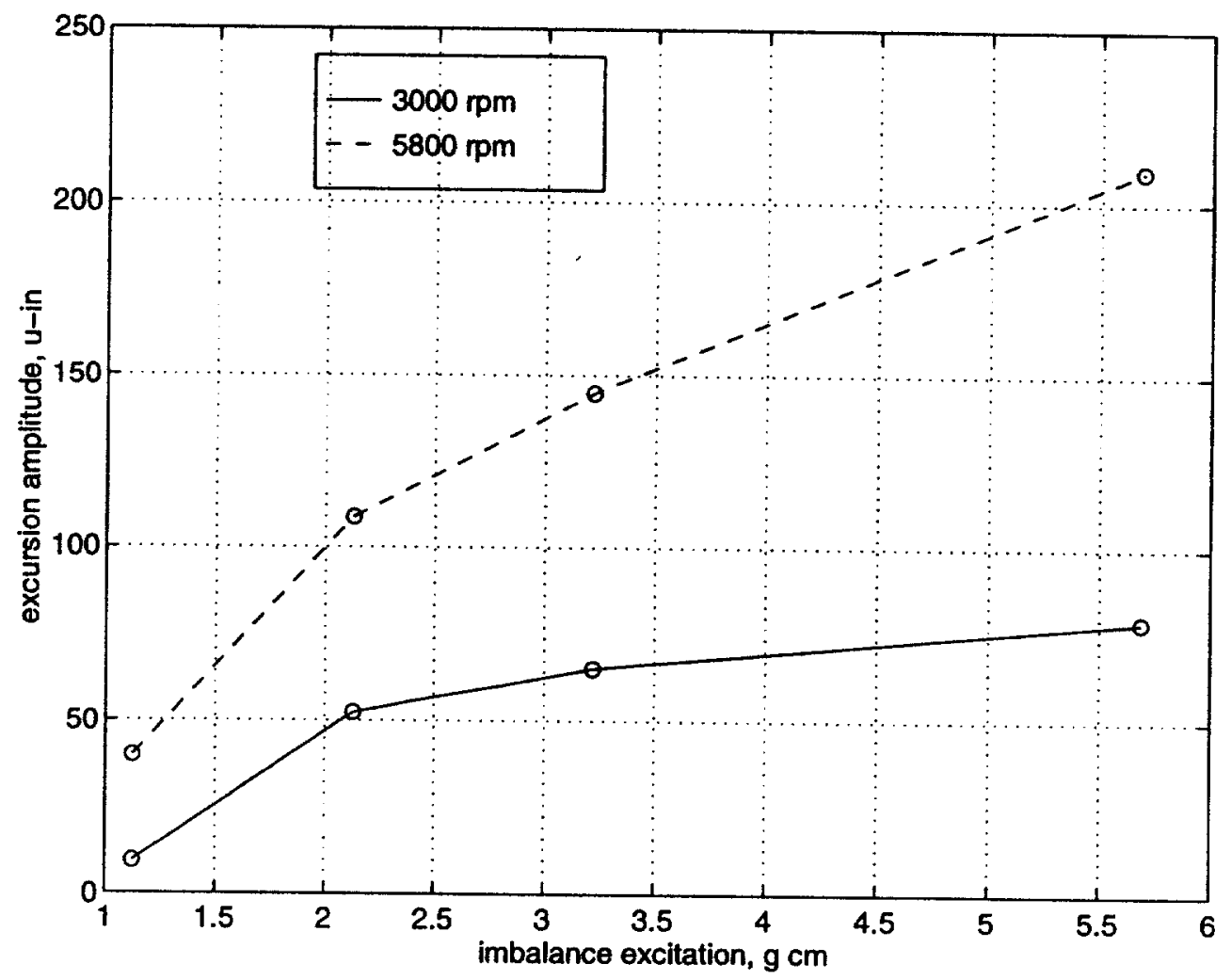

Figure 6-17: Effect of increasing mass of imbalance on error motion in the Y-direction, at two speeds. 


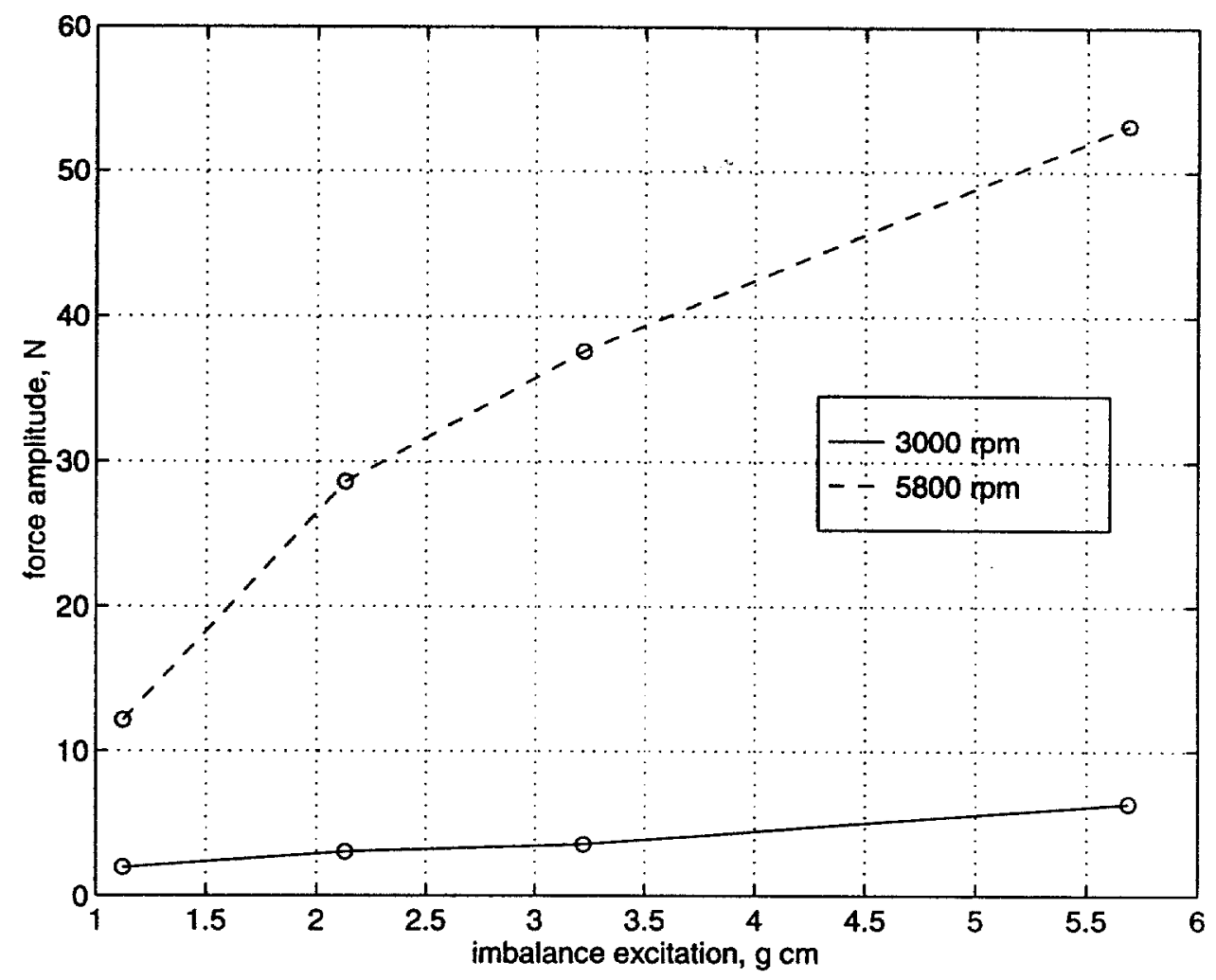

Figure 6-18: Effect of increasing mass of imbalance on force in the Y-direction, at two speeds. 


\section{Chapter 7}

\section{Experimental Challenges}

The purpose of this chapter is to discuss some of the challenges I faced in conducting the research presented. Since most theses and technical papers present their research as if it had been perfect all along, there is no opportunity for others to learn from the mistakes of the researchers. This chapter picks out several of the challenges I faced while conducting the experiments so that others may learn from them.

\section{1 "Noise" and Natural Modes}

A major problem which stymied the acquisition of spindle data with the Westwind spindle was "noise" in the load cell data. This problem took weeks to solve. Every time the spindle was powered up, the load cell would register a good deal of noise, on the order of 1.5-2 volts in amplitude, surrounding the DC offset voltage. To combat the problem I tried a variety of potential remedies. First, I talked to the supplier of the load cell (JR3) and asked about electrical noise isolation. Their load cell amplifier was equipped with three different electrical grounds, each referenced differently. Their technical support did not know which ground wire to use in which application, so I tried all three separately. These did not seem to change the output at all. Next, I looked at the power supply we were using for the JR3 amplifier. I compared the ground of that power supply to the electrical ground of the A/D board and to the electrical ground of the room, and I got a 5-10 millivolt difference. Thinking that differences in ground were a problem, I rearranged the experimental setup so that all of the instruments were powered off of the same circuit and dropped the ground differential to 2 millivolts. The problem persisted. 
Next I looked at the cabling from the JR3 load cell to the amplifiers and through to the computer. Thinking that electrical noise was being introduced through the cables, I used aluminum foil to shield the cables, but to no avail.

Since JR3 could not tell me whether or not the load cell should be electrically isolated from the system to which it was attached, I went ahead and tried isolating them. We used a piece of circuitboard material, cut out for the mounting holes, in between the spindle and the top mounting plate. (See figure 3-1.) In addition we isolated the mounting bolts from the spindle using plastic sleeves and washers. This reduced the noise somewhat, but there was still about \pm 1.5 volts of noise with the motor running.

I also tried combinations of shunt resistors at the input to the A/D board, at the suggestion of someone experienced with data acquisition. I shunted across from the instrument negative to ground with a $10 \mathrm{k} \Omega$ resistor, and this yielded the best results up to that point, although there was still an unacceptable level of electrical signal oscillation with the spindle powered up.

The problem was not solved until a site visit by my thesis advisor, who identified the cause immediately, and made it a major basis of this thesis. Reasoning that the "noise" was not at either the $60 \mathrm{~Hz}$ (power supply switching frequency) or $18 \mathrm{kHz}$ (Volkmann drive PWM switching frequency), he hypothesized that it was not electrical at all, but mechanical. To test his hypothesis, he looked at the JR3 outputs with an oscilloscope while he tapped the spindle with a screwdriver. The ringdown of the spindle after the excitation was identical in magnitude and frequency to the "noise". We had been observing natural modes of the system, excited by the powered rotation of the motor, and not electrical noise at all. It is postulated that the high-speed switching of current in the electric motor coil was creating a force oscillation which excited the system mechanically. (As current is passed through a coil, force $(F=i \times B)$ acts to drive apart neighboring windings.) When the motor was powered down, the mechanical oscillations rang down quickly, even as the spindle continued to rotate at speed.

This discovery had two effects. The first dealt with taking meaningful spindle motion data with the knowledge that modes were being excited in the spindle-load cell system by the motor. This meant taking data at speed with the motor powered off, since the modes would ring down almost immediately without motor power. To accomplish this, we eliminated the electric braking routine from the spindle controller, so that the spindle would 
spin down freely when stopped. Being an air-bearing spindle with large rotary inertia and low frictional dissipation, the natural spin-down time without braking is on the order of 5 minutes from $10,000 \mathrm{rpm}$. To take spindle data, I spun the spindle to about $1 \%$ above the desired speed, cut the controller power, and took the data after about a second of spin-down.

The far more important effect, however, was that understanding the modal behavior of the test setup became of paramount importance. Since production shops and machine designers use load cells to measure cutting parameters in-process, the dominant effect of spindle modes in a system such as this is of great concern. This same effect had been discovered by others at LLNL using a similar Westwind spindle and the same JR3 load cell earlier in the year: the system had large natural-frequency oscillations (although this was not understood at the time), and produced parts of poor quality when it produced parts at all. The load cell idea was scrapped in that particular setup due to instabilities which were ostensibly caused by natural frequency oscillations of the load cell-spindle system. In an industrial machining center, it is important that care be taken to avoid this sort of problem. There are many possible solutions, including using a stiffer load cell or adding damping material to the system to reduce unwanted oscillations. Any of these fixes may reduce vibrations to an acceptable level while preserving the ability to measure machining forces in-process with a load cell.

In the case of this particular experimental setup, I decided to understand the modal behavior as completely as possible in the remaining months. First, I obtained a spectrum analyzer which would allow me to look at the output of the JR3 load cell in the frequency domain. I watched as five dominant peaks, resonances of the system, shifted in magnitude with different rotational speeds of the spindle. (I could also see electrical peaks at $60 \mathrm{~Hz}$ and $18 \mathrm{kHz}$.) These were the fundamental modes of the system, and they occurred between 100 and $200 \mathrm{~Hz}$. To further understand to which oscillatory modes these natural frequencies corresponded, I undertook a rudimentary modal analysis. Using the JR3 load cell as a force sensor, and looking at outputs in each of the three force and moment directions with the spectrum analyzer, I excited the spindle with a plastic hammer and recorded the spectra. Each spectrum was an average of ten "grabbed" spectra from a hammer strike. By exciting the spindle in a variety of different locations, I was able to obtain different spectra which could be compared, in each of the sensing directions. Figure 7-1 shows the spectra for excitation of the spindle at the nose as sensed by the load cell in the X, Y, and Z-directions. 


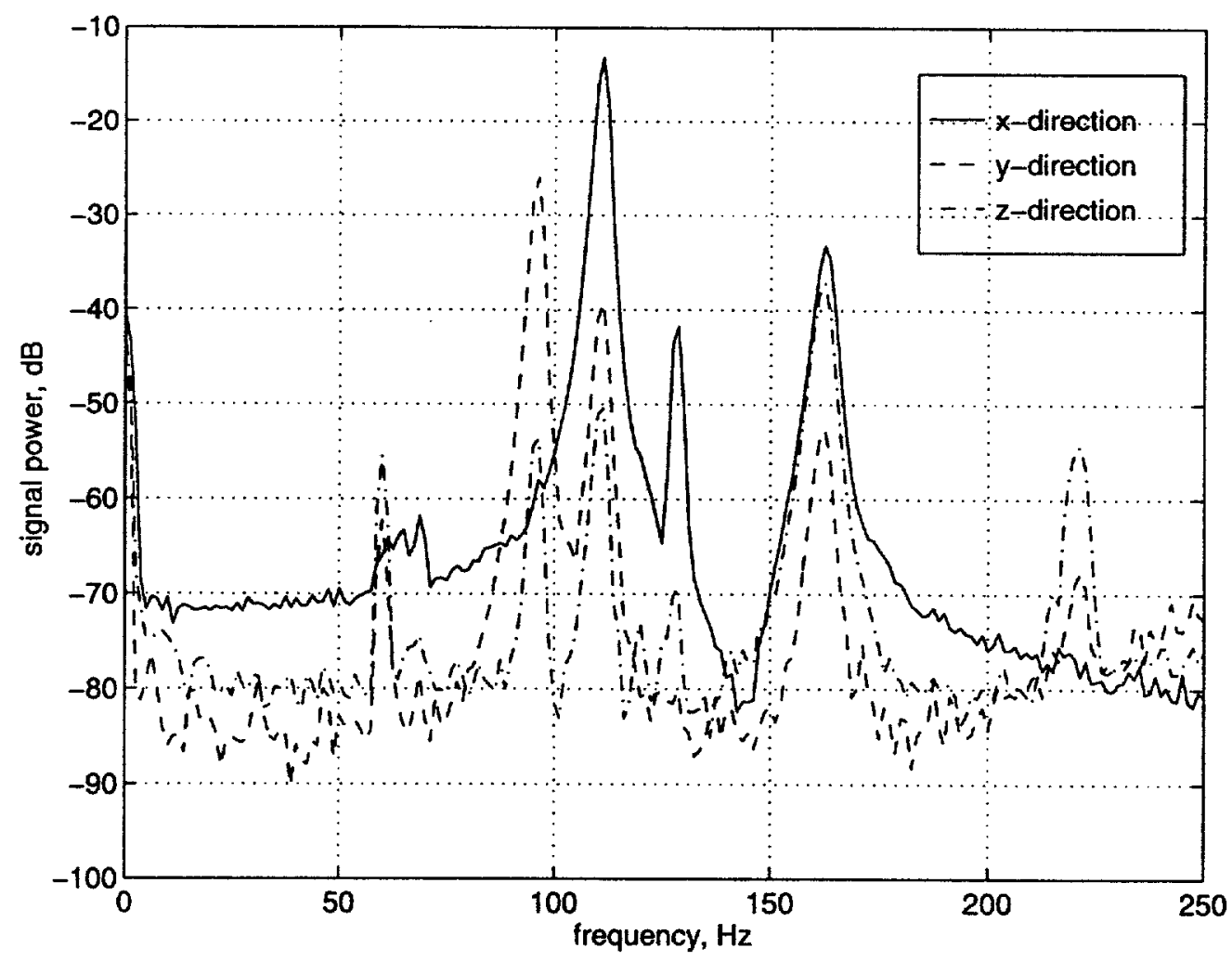

Figure 7-1: Sample graph of frequency domain output for load cell; spindle excited at nose and sensed in each of three directions. (Directions are as defined in figure 3-1, with $\mathrm{x}$ and $y$ interchanged.) 
I repeated this rudimentary modal test using the stiffer Kistler $9257 \mathrm{~A}$ load cell. $\mathrm{My}$ thought was that some effects would be inherent to the Westwind spindle itself and hence "load cell independent." By testing with two different load cells, I could observe what these effects were. (The original reason for adding the Kistler load cell was to see if the number of system modes would decrease with that setup.) In reality, each setup proved to be its own global system. In modal tests with the load cell in place, many rigid-body modes were apparent. In the test with just the spindle bolted to the granite block, bending modes which took place within the spindle itself were much more apparent.

Using the frequency spectra obtained with different excitation points on the setup, I attempted to deduce mode shapes for the system. I looked at peaks which occurred on all the spectra and compared their magnitudes to come up with a hypothesis that explained each peak. For example, if a peak featured prominently in the Z-direction, but not the $\mathrm{X}$ - or Y-directions, I would hypothesize that the mode associated with that frequency was predominantly translation in the Z-direction. I then looked at the three moment direction spectra to see if the frequency showed up there, as well. Many times there would also be a rotational component to the mode. This worked very well; I was able to come up with simple explanations for the prominent natural frequencies for each setup. My hypotheses were later validated and expanded by the more rigorous STAR modal tests.

Another upshot of the discovery of important natural modes in the system was the addition of damping material to the JR3 setup. I tried adding damping to see if I could in practice lessen the effect of the modes on spindle error. This modification would have applications if this or a similar setup were used in a machining operation at the Laboratory. I added thin strips of Sorbothane-30 material between the fixture plates in parallel with the load cell, along the length of the fixture plates on two sides. I ran the imbalance tests again with the damping material in place.

Ultimately, the discovery of natural modes led to a full modal test of the four systems (spindle-JR3, spindle-JR3-damping, spindle-Kistler, and lone spindle) using STAR modal analysis software. This testing provided the greatest insight into how the system oscillates at various frequencies and gave animated mode shape data and the ability to simulate arbitrary force inputs to the system, both of which are of great use to this project. 


\subsection{Clock Speed Differences}

In taking the simultaneous load cell and spindle analyzer data with the two CPUs (as described in Chapter Two) one problem we encountered was differences in the counter pulses observed by each system. The counter pulse is the once per revolution signal from the rotating timing wheel, as observed by the photoeye. We gathered counter pulse data on each of the three A/D boards used in the data collection. Since the systems started taking data at the same instant (within \pm 40 microseconds of each other) the reference pulses on the datafiles initially lined up. However, after about a second of data collection, the pulses from the two systems had drifted apart, with the Pentium pulse leading the two Tektronix pulses by around $1 \%$.

This differential can be attributed to different clock speeds between the two systems. In most applications, the user of a computer does not care if his clock is running at $25 \mathrm{MHz}$ or $25.1 \mathrm{MHz}$, and so the manufacturers of PCs do not put much effort into using accurate clock chips, or even making sure the clock speeds agree unit to unit. The drift between the two systems also varied: in some cases it was a $0.8 \%$ lead, in others a $1.3 \%$ lead. It was instructive to me to learn that this potential discrepancy exists in computer clocks; this has implications not only in the kind of synchronization problems we faced in this project but also potentially in applications like digital control. In our application, the problem is relatively easy to fix after the data has been gathered, by measuring the offset on MATLAB or a similar program and scaling one set of time data so that the two signals match.

\subsection{Metrology Ball Drift}

When taking the first sets of spindle and load cell data, I checked the position of the metrology ball at the outset of each run. I rotated the spindle by hand and looked at the maximum capacitance gauge offset and its position relative to the timing window. This number varied between each run. Sometimes the drift from run to run was fifty microinches or more. This meant that ball offset data could not be trusted, even within the same run. Next I tried running the spindle at high speeds for long periods (order of hours) to see if the ball offset would "seat" in one place. This worked in the short term, but over time (overnight) thermal drift would move the ball to a new location.

Next I looked at the metrology ball setup with the help of a machinist. We noticed that 
simply tapping the fixture lightly with a small screwdriver would cause the ball to move erratically, by as much as 100 microinches. Examining the ball fixture, we found a precision metrology nightmare - there were three attachment systems in use simultaneously. The ball shaft was attached to the spindle through a small aluminum collet, into a brass slip ring, and finally into a the $5 / 8^{n}$ Westwind spindle collet. The high number of degrees of freedom made ball accuracy impossible.

The solution to this problem is the fixture presented in figure 3-2. This fixture consists of a $5 / 8^{\prime \prime}$ sleeve for the metrology ball shaft which fits directly into the spindle collet. The new fixture preserves the flexural adjustment of the ball center found in the original fixture, and it eliminates two extra collets in the original system. Performance with the new fixture is markedly better, with drift between tests on the order of two microinches, and consistent angles of offset. The thermal drift problem also improved significantly with the new fixture. This experience taught me the importance of thinking through each assembly in a precision machine and making sure that it is properly constrained.

\subsection{Spindle Analyzer and Anti-Aliasing Filter}

Before the development of the new $\mathrm{C}$ code and synchronization routine for taking spindle analyzer data (which is described in Chapter Two of this thesis), I was attempting to use a spindle analyzer program that had been developed at the Laboratory five years before. This program had originally been implemented in PASCAL, and then converted into $\mathrm{C}$ code, and since then had been modified by three other users. It ran on a PC and took data from the capacitance gauges through an $\mathrm{A} / \mathrm{D}$ board. It had functions for displaying the resultant spindle analyzer data in polar form and calculating relevant spindle error parameters. (For example, asynchronous axial and radial error motion, as described in the ANSI Axis of Rotation Standard, [7].) Originally I was planning to use this software to acquire data for my experiments.

At some rotational speeds, however, this software produced erratic results. It would display a jagged "triangle wave" or yield outputs which were unreasonably low in error magnitude (for example, one microinch of radial error at 9,000 rpm, for a setup that had a known once per revolution error of 50 microinches). However, this occurred only at multiples of $3,000 \mathrm{rpm}$ in spindle speed. Previous users of the software advised me to "avoid 
those speeds." At other speeds the software performed consistently, producing believable error plots. I read through the source code for the program, and could not find anything glaringly wrong. I decided to check into sampling rate issues, since the errors appeared to be so closely coupled with rotational speed. The software picked a sampling rate based on fifty data points per revolution and real-time measured rotational speed, based on the counter pulse output. It then commanded the A/D board to sample at this rate and took the data. However, it also wrote half of the sampling rate (the Nyquist frequency) to an address that anti-aliasing filters read. These filters were located external to the rest of the data acquisition system, inside the box which contained the capacitance gauge amplifiers.

Next I found that the make and model on the actual filters did not match the one given in the program. After talking to the manufacturer of the filters, I realized that an entire section of code had been written for another filter and never changed. Each filter was setting its anti-aliasing cutoff at tens of times the desired cutoff, and so high-frequency data (which is plentiful in a rotating spindle!) was being aliased down and misinterpreted by the software. A basic change in the code fixed the problem.

We did not use this code for our spindle analysis in the end due to problems of synchronization with the Tektronix system. However, the experience pointed out the importance of knowing exactly how data is processed at each step in a data acquisition system, and in a larger sense the importance of understanding as completely as possible any tool used in engineering. 


\section{Chapter 8}

\section{Recommendations}

\subsection{Summary of Results}

In the chapter "Project Description", I set up a series of questions relating to balance in grinding that could be asked by a machine or process designer. These were:

- "How fast should I cut?"

- "What difference does a 'softer' or 'stiffer' machine make?"

- "How are wheel truing and balance related?"

- "How does error motion scale with imbalance magnitude?"

- "If I add damping, will that improve the spindle error motion due to imbalance?"

This thesis attempts to answer these questions through experiment and modeling. The first question, "How fast should I cut?", can best be answered by understanding the resonant frequencies of the machine structure. Assuming that large spindle error motion should be avoided, the speed of the grinding wheel should be chosen such that it is far away from resonances in the sensitive direction of cutting. In the Westwind-load cell systems described in this thesis, each direction (X, Y, and Z) had its own set of resonant peaks, sometimes at very different frequencies. Depending on the direction of concern in cutting, the spindle speed can be chosen so as to avoid these resonances. Determining machine resonances can be done in many ways: in this thesis I explored the relatively time-intensive capacitancegauge method of spindle characterization. In addition, I looked at a much quicker method: 
empirical modal analysis. From either of these methods, it is relatively easy to pick an operational speed for a system which avoids large oscillation due to structural resonance.

The second question, "What difference does a 'softer' or 'stiffer' machine make?", was explored by comparing the Westwind-JR3 system to the Westwind-Kistler system. With the softer JR3 system, there were more structural resonances and spindle error motion was in general larger than with the stiffer Kistler system. However, the Kistler system also transmitted more force oscillation due to imbalance through to the external structure (in this case the isolated granite block) than did the JR3. This result can be extended to other grinding systems: if the underlying structure had been a conventional machine tool, rather than an enormous granite block, it would have been subjected to larger force amplitudes with the stiffer load cell. This force oscillation could excite additional machine dynamics in the structural loop and contribute to error motion through that route. In the more general case of machine design, however, the advantages of a stiff machine over a softer one are obvious. A stiffer machine will reduce the spindle error motion due to imbalance, geometric eccentricity of the wheel, or other error sources.

The next question, "How are wheel truing and balance related?", was explored with a series of tests which used an offset metrology ball as an "untrued" grinding wheel and an imbalance mass placed in varying angular positions relative to the decenter. With the imbalance mass and decenter co-located angularly, the effects compounded, leading to a more pronounced error motion. As the mass was rotated relative to the decenter, however, the amount of error motion lessened. And at one location the imbalance did compensate out some of the decenter. One of the central developments in this work is explicitly separating these two effects, which are often confused in practice. These tests showed that the effects are indeed separate, and either reinforce each other, increasing error motion, or cancel each other, lessening error motion. This has some potential applications in the truing of grinding wheels: if it is possible to add a small amount of imbalance to a grinding wheel to offset a small truing error this may save process setup time greatly. This method is already used in the manufacture of large grinding wheels: once the center hole is drilled, material is removed to change the balance of the wheel until the wheel runs true. This idea does not seem to be applied yet in precision grinding, however.

"How does error motion scale with imbalance magnitude?" was investigated by increasing the mass of imbalance and watching how both error motion and force transmitted to 
the machine in one direction changed. From the results, there is a logarithmic shape to the relationship between both magnitude of imbalance and force transmitted to the surrounding machine as imbalance mass increases.

The final question posed was, "If I add damping will that improve the spindle error motion due to imbalance?" After tests on the JR3-Westwind system, the answer is definitely yes. The addition of damping to that system, across a critical gap of machine motion, greatly improved the error motion due to imbalance. In these tests, that fix was very quick: a backof-the-envelope calculation of an appropriate damping material thickness, and addition of the material to the system on top of boxbeam. This reduced displacement oscillation due to imbalance by a half or better in some cases. The addition of damping flattens out resonant peaks in the frequency response of the machine, making for a more homogenous frequency response curve. It would seem that most precision machine tools would benefit greatly from the intelligent addition of damping material. Vibrating energy which is dissipated in damping material is energy that is not carried through the cutting process to the part surface!

\subsection{Extension of Study}

There are many paths to pursue to carry this study forward. One potentially very interesting experiment would be to perform empirical modal analysis on a large machine tool, such as one of the air-bearing grinders set up on a T-base foundation at Lawrence Livermore. By using the modal data to predict system resonances in the sensitive direction of grinding, grinding speeds which were sensitive to imbalance of the spindle could be predicted. Then these predictions could be tested by measuring the error motion of the spindle across its range of operating speeds. Even more interesting would be the correlation of part surface roughness across the range of cutting speeds with modal data for the structure. This type of experiment would yield insight into the importance of balance as it relates to both surface finish and cutting speed. It would also validate the idea that modal analysis can be used as a cheaper alternative to more costly spindle analysis methods for quantifying error motion of a machine at various speeds.

Another set of experiments which would be an excellent complement to this thesis would be the investigation of decenter versus imbalance in a commercial precision grinding 
machine. This set of tests could consist of two parts: the first part showing that truing error can be compensated by carefully-chosen imbalance and the second part investigating the quality of work produced by a traditionally trued and balanced system and the new unbalanced system. This would show the feasibility of such a procedure in precision grinding applications. Additionally, the closed-loop use of a dynamic balancer to compensate for force oscillation on the part (as opposed to shaking of the spindle stator) could be investigated.

One very useful set of experiments would be to validate the dashpot model ( [8]) of the grinding process over a frequency range. In the general one-dimensional model presented in this thesis, the dashpot is assumed to be good over all frequencies. In practice, however, the interaction between grinding wheel and workpiece could potentially be much more complicated. The dashpot model may hold at low frequencies, with the Hertzian-contact stiffness between the wheel and workpiece dominating at higher frequencies, for example. To explore these ideas, a series of experiments with an instrumented workpiece and varying grinding wheel rotational speeds (exciting the system at different frequencies) and varying infeed speeds could be undertaken. The results from these experiments would be a step towards formulating a robust cutting process model.

Further modeling work could also be undertaken, to develop a lumped-parameter model of a grinding machine like the one presented in this thesis. An enhanced model could use well-measured parameters from a commercial grinding machine as its process parameters. It could be extended to multiple dimensions and degrees of freedom from the model presented here. The goal of this model would be to simulate a real grinding machine and predict the magnitude of relevant variables of the grinding process. With the level of understanding from a robust, validated model would come many advantages. Effects of many types of error sources (i.e. balance, decenter, thermal errors, seismic errors) could be studied, and error budgeting for the system vastly improved. In addition, having a robust model of a large grinder would have potentially rewarding applications in active control of the grinding process instead of the open-loop grinding most common today. (See [9].)

Very helpful in any work of this sort would be a model which predicts part surface finish based on wheel geometry, wheel speed, part composition, input grinding force, and input spindle error motion. Then force and error motion could be input from a separate model (akin to the general model presented in this thesis) and part surface predicted using the cutting model. The model would ideally be able to be modified for any of a number of 
machine. This set of tests could consist of two parts: the first part showing that truing error can be compensated by carefully-chosen imbalance and the second part investigating the quality of work produced by a traditionally trued and balanced system and the new unbalanced system. This would show the feasibility of such a procedure in precision grinding applications. Additionally, the closed-loop use of a dynamic balancer to compensate for force oscillation on the part (as opposed to shaking of the spindle stator) could be investigated.

One very useful set of experiments would be to validate the dashpot model ( [8]) of the grinding process over a frequency range. In the general one-dimensional model presented in this thesis, the dashpot is assumed to be good over all frequencies. In practice, however, the interaction between grinding wheel and workpiece could potentially be much more complicated. The dashpot model may hold at low frequencies, with the Hertzian-contact stiffness between the wheel and workpiece dominating at higher frequencies, for example. To explore these ideas, a series of experiments with an instrumented workpiece and varying grinding wheel rotational speeds (exciting the system at different frequencies) and varying infeed speeds could be undertaken. The results from these experiments would be a step towards formulating a robust cutting process model.

Further modeling work could also be undertaken, to develop a lumped-parameter model of a grinding machine like the one presented in this thesis. An enhanced model could use well-measured parameters from a commercial grinding machine as its process parameters. It could be extended to multiple dimensions and degrees of freedom from the model presented here. The goal of this model would be to simulate a real grinding machine and predict the magnitude of relevant variables of the grinding process. With the level of understanding from a robust, validated model would come many advantages. Effects of many types of error sources (i.e. balance, decenter, thermal errors, seismic errors) could be studied, and error budgeting for the system vastly improved. In addition, having a robust model of a large grinder would have potentially rewarding applications in active control of the grinding process instead of the open-loop grinding most common today. (See [9].)

Very helpful in any work of this sort would be a model which predicts part surface finish based on wheel geometry, wheel speed, part composition, input grinding force, and input spindle error motion. Then force and error motion could be input from a separate model (akin to the general model presented in this thesis) and part surface predicted using the cutting model. The model would ideally be able to be modified for any of a number of 
grinding configurations - cup grinding, peripheral grinding, face grinding, etc. Currently the grinding models which exist are very application-specific and not easily modified to solve general problems. Having a robust cutting model would be of great help to precision grinding research.

\subsection{Industrial Applications}

There are a number of potential industrial applications from the work presented in this thesis. One is that empirical modal testing is a potentially useful alternative to other types of spindle error motion analysis. It has the advantage of being much faster than capacitance gauge testing and provides good insight into the dynamics of the machine tool. With understanding of the dynamics comes an intelligent choice of process speed and perhaps good guidelines for the amount of imbalance and decenter which can be tolerated in the process. Further, this thesis has demonstrated that knowledge derived from the modal testing can be used to produce useful analytical and theoretical models of the machine tool. As the tolerances in precision grinding get ever smaller, it will be of paramount importance to understand the dynamics of the system as fully as possible.

Another potential application is compensation of truing error with chosen imbalance. In precision grinding, much time is spent in getting the wheel trued to an acceptable level of error. With electro-discharge machining methods and a bronze-bond wheel this can sometimes take on the order of days. The judicious addition of a small imbalance could correct the truing error with minimal effect on the part. This method is already being used in the manufacture of grinding wheels; it should be attempted in precision grinding. If found to be practical, it could save a great deal of time in process set up.

Finally, it has been shown here that adding damping to a grinding system can greatly reduce spindle error motion due to imbalance. This could translate into improved process quality with no adverse effects. This is another potential solution to the problem of imbalance in grinding. By flattening out the frequency response spectrum of the machine, the effects of resonant frequencies can be lessened. Many existing precision grinding machines could benefit from the careful addition of damping material. It is helpful in dissipating energy from diverse error sources that would otherwise go into the cutting process. 


\section{Appendix A}

\section{MATLAB Script for General Grinding Model}

function [out1, out2, out3] $=\operatorname{Grind10}(t, y)$

$\% \% \% \%$ SOLUTION PARAMETERS $\% \% \% \% \%$

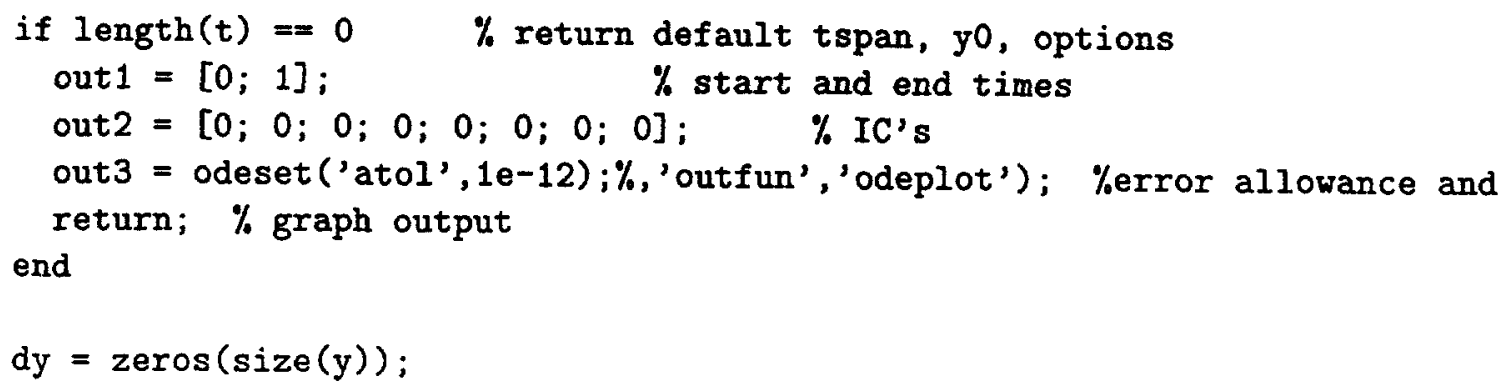

$\% \% \% \%$ DEFINITION OF VARIABLES $\% \% \% \%$

$\%$ component

$\%$ machine mass 1

$\%$ wkpc spindle mass

$\%$ tool spindle mass

$\%$ machine mass 2 position

$\mathrm{y}(1,:)$

$\mathrm{y}(2,:)$

$\mathrm{y}(3,:)$

$y(4,:)$ velocity

$\mathrm{y}(5,:)$

$y(6,:)$

$\mathrm{y}(7,:)$

$y(8,:)$

$\% \% \%$ ELEMENT PARAMETERS $\% \% \% \%$

$M 1=2000 ;$

M2 $=1000 ; \% 500 / 2.2$ for Moore

M3 $=25.36 / 2.2$;

$M 4=2000$; 


$$
\begin{aligned}
& \mathrm{K} 1=12.8 \mathrm{e} 6 ; \\
& \mathrm{K} 2=1 \mathrm{e} 5 ; \\
& \mathrm{K} 3=57.8 \mathrm{e} 6 ; \\
& \mathrm{K} 4=57.8 \mathrm{e} 6 ; \\
& \mathrm{B} 1=1 \mathrm{e} 5 \\
& \mathrm{~B} 2=1 \mathrm{e} 5 \\
& \mathrm{~B} 3=1 \mathrm{e} 5 ; \\
& \mathrm{B} 4=0 ; \% \text { see below }
\end{aligned}
$$

$\% \% \%$ CUTTING MODEL PARAMS \%\%\%\%

rpm $=5000 ; \%$ grinding spindle speed

$A=1 e-6 ; \%$ size of contact patch

$\mathrm{Kp}=0.2 \mathrm{e}-4 ; \%$ Preston coefficient (should itself be a function)

$\mathrm{V}=(\mathrm{rpm} / 60) *(\mathrm{pi} * 0.30) ; \%$ relative velocity of surfaces

Fth $=0 ; \quad \%$ threshold grinding force for mat'l removal, assumed zero

$\%$ since we're already grinding

$\mathrm{Bp}=\mathrm{A} /(\mathrm{Kp} * \mathrm{~V})$;

$F b p=B p *(y(7,:)-y(6,:))+F t h ;$

$\% \% \% \%$ FORCE INPUTS $\% \% \% \%$

$\mathrm{Me}=.016$

$\mathrm{e}=0.10$;

omega $=\mathrm{rpm} *(2 * \mathrm{pi} / 60)$;

Fbe $=$ Me*e*omega ${ }^{\wedge} * \sin ($ omega $* t) ;$

$\operatorname{del} \mathrm{r}=100 * 2.54 \mathrm{e}-8$;

phi $=0 *(2 *$ pi/360); \%phase lag between peak imbalance and peak geo. eccent. ygedot $=$ delr*omega*cos (omega*t + phi); \%deriv of fxn describing geom error $\%$ displ

Fge $=0 ; \%($ ygedot $* B p)+F$ th

$\% \% \% \%$ ELEMENT FORCE RELATIONS \%\%\%\%

$\mathrm{Fk} 1=\mathrm{K} 1 *(\mathrm{y}(1,:)-\mathrm{y}(4,:)) ;$

$F k 2=K 2 *(y(4,:))$;

$\mathrm{Fk} 3=\mathrm{K} 3 *(\mathrm{y}(1,:)-\mathrm{y}(2,:))$;

Fk4 $=K 4 *(y(3,:)-y(4,:))$;

$\mathrm{Fb} 1=\mathrm{B} 1 *(\mathrm{y}(5,:)-\mathrm{y}(8,:)) ;$

$\mathrm{Fb} 2=B 2 *(y(8,:))$;

$\mathrm{Fb} 3=\mathrm{B} 3 *(\mathrm{y}(5,:)-\mathrm{y}(6,:)) ;$

$\mathrm{Fb} 4=0 ; \% \mathrm{~B} 4 *(y(7,:)-\mathrm{y}(8,:))$;

$\% \% \%$ SYSTEM EQUATIONS $\% \% \% \%$ 


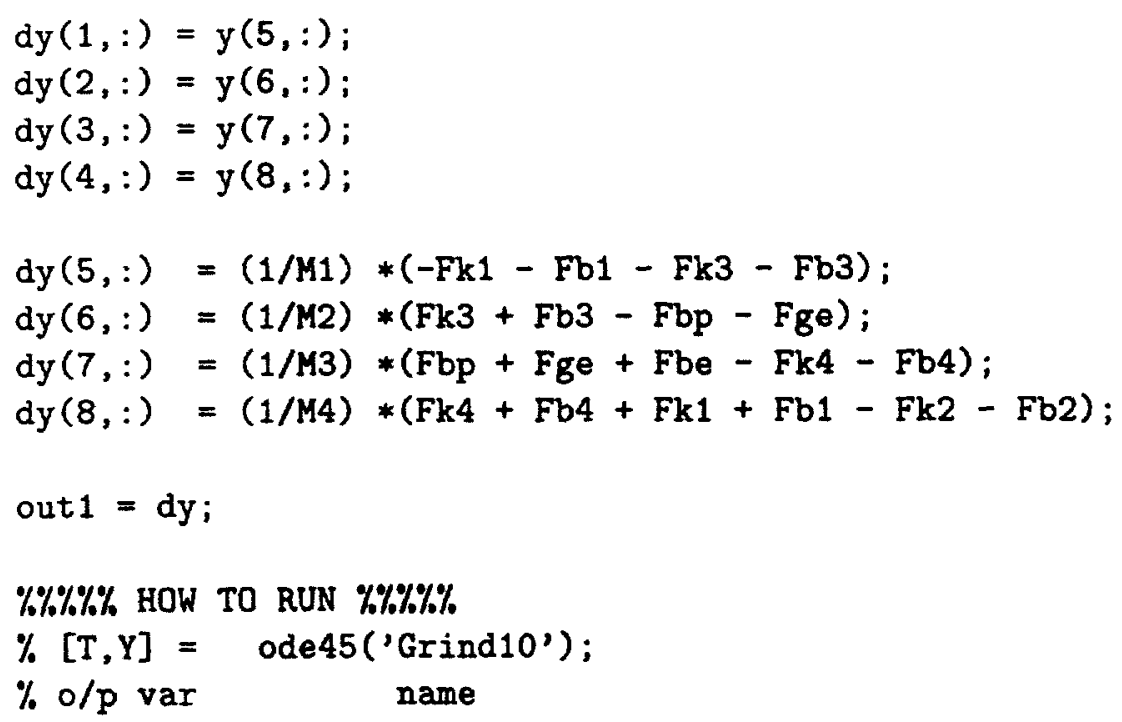




\section{Appendix B}

\section{MATLAB Script for Analytical Grinding Model}

\section{B.1 ODE solver}

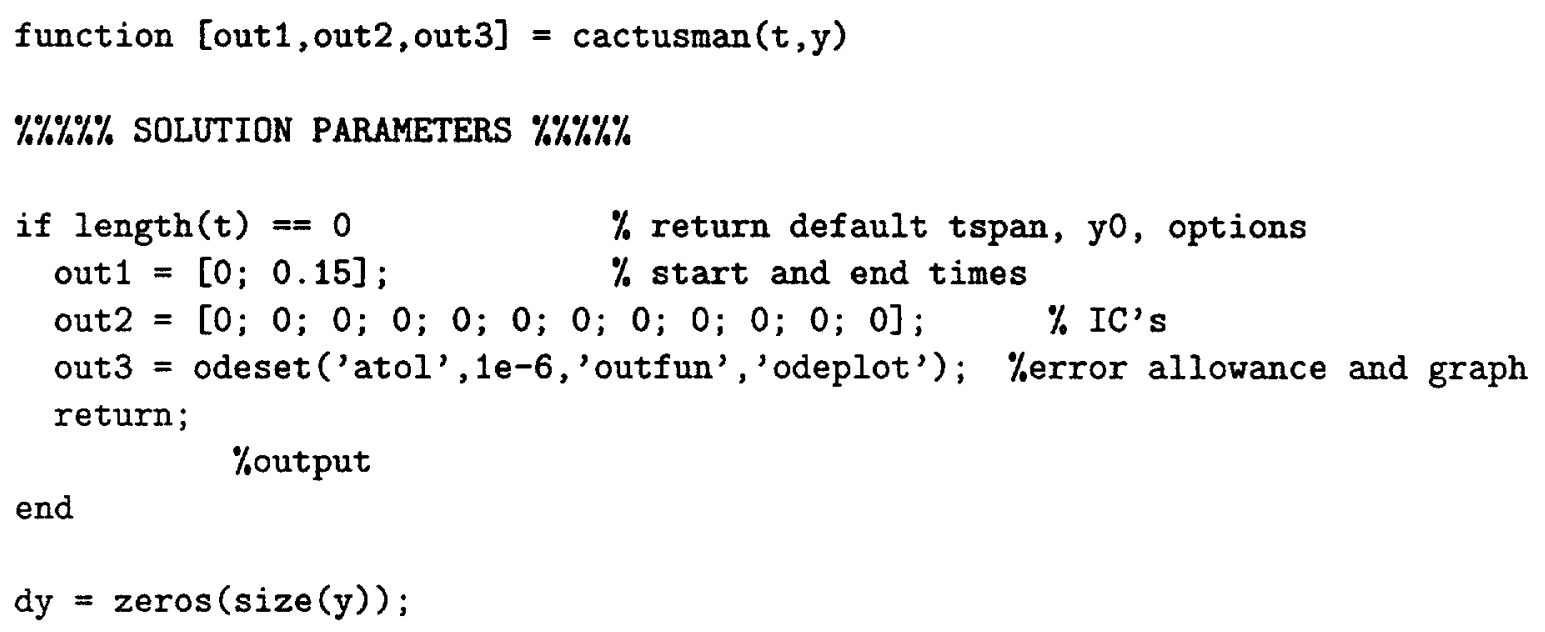

$\% \% \%$ ELEMENT PARAMETERS $\% \% \% \%$

$I 1=10.41$; 


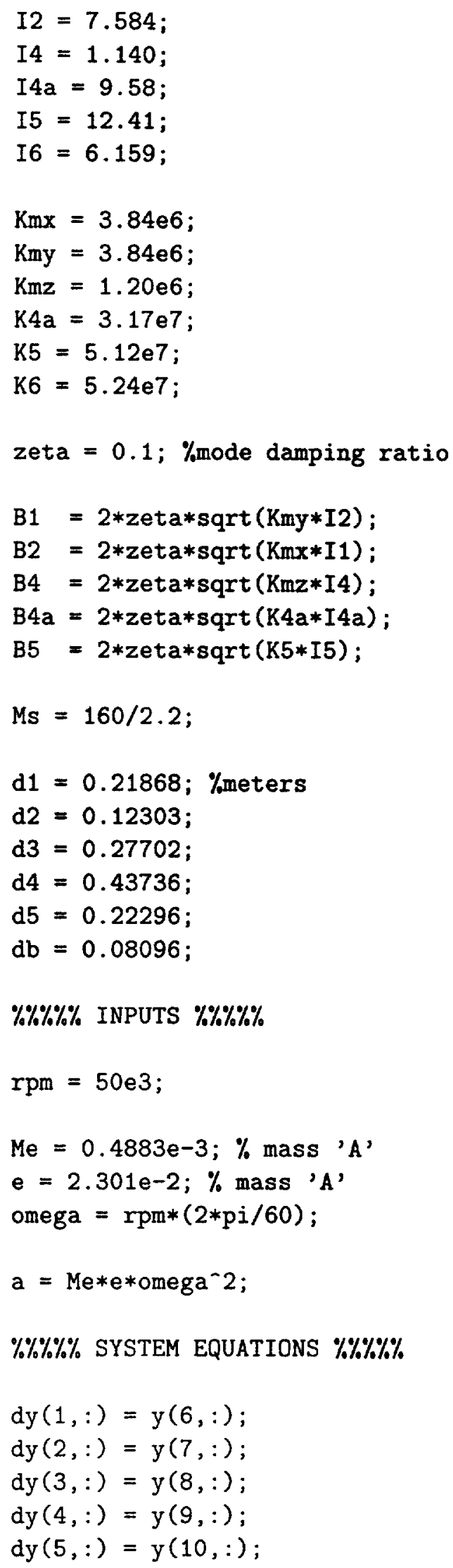

$\% \% \%$ SYSTEM EQUATIONS $\% \% \% \%$

$\operatorname{dy}(1,:)=y(6,:)$;

$\operatorname{dy}(2,:)=y(7,:)$;

$\operatorname{dy}(3,:)=y(8,:)$;

$\operatorname{dy}(4,:)=y(9,:)$;

$\mathrm{dy}(5,:)=\mathrm{y}(10,:)$; 
$\operatorname{dy}(6,:)=(1 / I 2) *(-K m y * y(1,:)-B 1 * y(6,:)+a * \sin ($ omega*t $+3 * p i / 2) * d 1) ;$

$\operatorname{dy}(7,:)=(1 / I 1) *(-K m x * y(2,:)-B 2 * y(7,:)+a * \sin ($ omega*t + pi $) * d 2)$;

$\operatorname{dy}(8,:)=(1 / I 4) *(-K m z * y(3,:)-B 4 * y(8,:)+a * \sin ($ omega*t $) * d 1)$;

$\operatorname{dy}(9,:)=(1 / 14 a) *(-K 4 a * y(4,:)-B 4 a * y(9,:)+a * \sin ($ omega*t $+3 * p i / 2) * d 3) ;$

$\operatorname{dy}(10,:)=(1 / I 5) *(-K 5 * y(5,:)-B 5 * y(10,:)+a * \sin ($ omega*t $+3 * \mathrm{pi} / 2) * d 4) ;$

out1 $=\mathrm{dy}$;

$\% \% \%$ HOW TO RUN $\% \% \% \%$

\%path ('/mit/case/thesis/MatLaB/odesuite',path)

$\%[T, A]=$ ode45 ('cactusman') ;

$\%$ o/p var name

$\%$ use the file post 2 .m to convert coordinates and add in ball decenter

\section{B.2 Post Processor}

$\%$ post-prccessor for cactusman.m model

$\%$ this routine will map the trans. and rot. deflections from the model into

$\%$ spindle error motion

$\% \% \% \%$ SYSTEM DIMENSIONS $\% \% \% \%$

$\mathrm{d} 1=0.21868 ; \%$ meters, make sure same as cactusman.m !

$\mathrm{d} 2=0.12303 ;$

$\mathrm{d} 3=0.27702$;

$\mathrm{d} 4=0.43736$;

$\mathrm{d} 5=0.22296$;

$\mathrm{db}=0.08096$;

$\operatorname{rad} 1=\left(\mathrm{d} 2 \_2+(\mathrm{d} 1+\mathrm{db}) \sim 2\right) \sim 0.5$

$\operatorname{rad} 2=\left((d 2-d 5)^{\wedge} 2+(d 3+d b)^{\wedge} 2\right) \wedge 0.5$;

ang1 $=\operatorname{atan}(d 2 /(d 1+d b))$;

$\operatorname{ang} 2=\operatorname{atan}((d 3+d b) /(d 5-d 2)) ;$

$\% \% \%$ INPUTS $\% \% \% \%$

$\mathrm{rpm}=50 \mathrm{e}$;

omega $=\operatorname{rpm} *(2 * \mathrm{pi} / 60)$;

driver $=\sin ($ omega $* T)$;

$\% \% \%$ OUTPUTS $\% \% \% \%$

ythetax $=-d 2 * \sin (A(:, 1))$;

zthetax $=-d 2 *(1-\cos (A(:, 1)))$; 


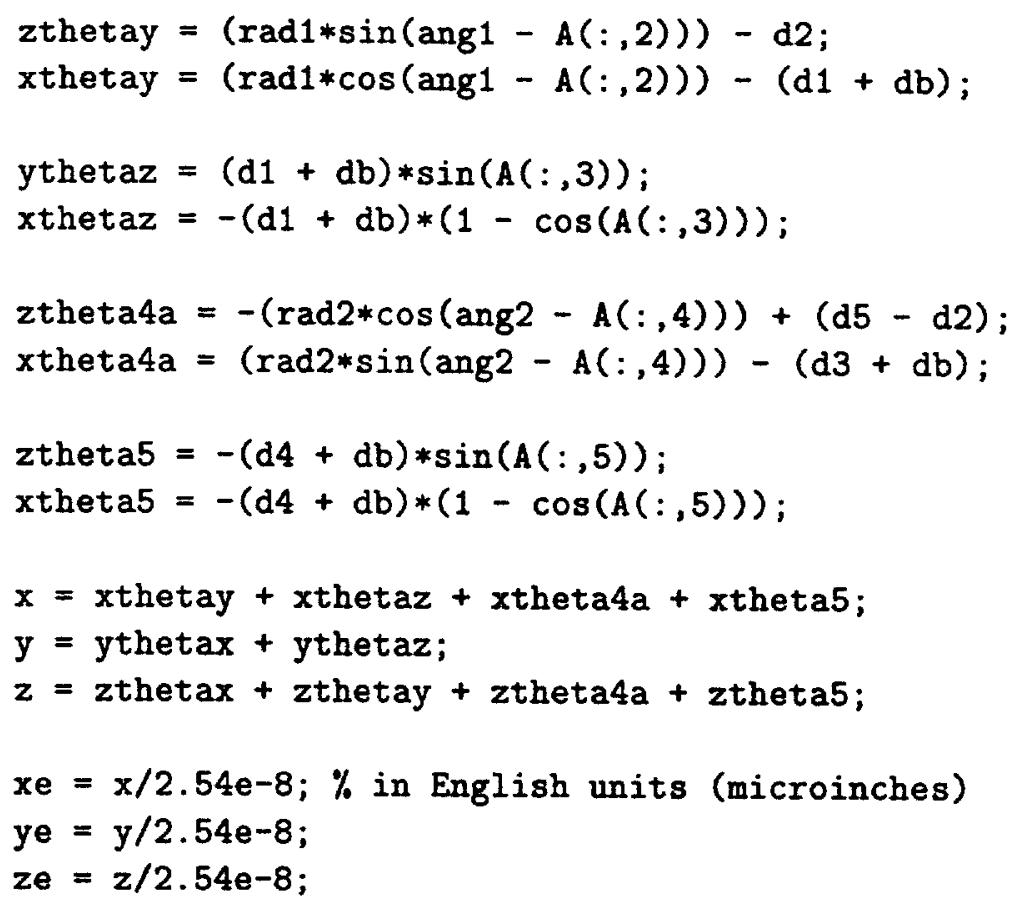




\section{Appendix $\mathbf{C}$}

\section{Data Reduction}

\section{C.1 MATLAB Procedure}

Each spindle test (approximately one second of data) was stored to its own data file (around 800 Kbytes of data) and the relevant information on rotational speed, amplitude, and phase of each relevant signal had to be extracted from this file. This was done graphically using MATLAB scripts which, after a data file had been loaded (as a large, multiple-column array), allowed the determination of the relevant parameters. The scripts allow the user to click on a region of the graph, zoom in, and then they output desired quantities after getting user input from the graph. Amplitude of the signal was found by taking half of the peak-to-peak value. Phase was found relative to the once per revolution counter pulse, and then later adjusted for phase relative to the desired sensitive direction. A portion of an example MATLAB script, for reduction of Kistler axial data, follows.

clear;

load ka39.dat;

$\mathrm{J}=\mathrm{ka} 39$;

$\% \%$ FREQUENCY DETERMINATION

$\operatorname{plot}(J(:, 7), J(:, 8))$; \%plot pulses

title('Choose frequency window:');

corner $=$ ginput $(3) ; \%$ this corner routine lets the user zoom in on a

$\%$ region by clicking on the bottom left, bottom right, and top corners axis ([corner (1,1) corner (2,1) corner $(1,2)$ corner $(3,2)]) ; \%$ reset the axis

title('Choose 5-tick span for frequency measurement:'); \% prompts next step 


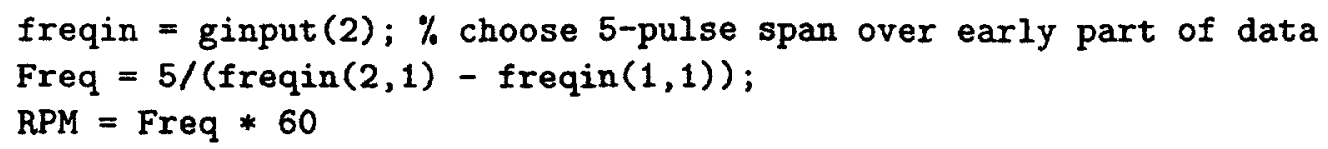

Similar MATLAB scripts enabled data reduction for the different types of data files. The data was then entered into arrays which were stored and graphed on MATLAB.

\section{C.2 Tehranchi's Identity}

Before each set of data was obtained, the amount of ball decenter and its phase were measured manually. This was for the purpose of correcting the data for the geometric offset, so that only error motion due to imbalance remained. To do this, one must apply Tehranchi's identity, which allows the subtraction of two sinusoids of the same frequency, but different amplitudes and phases.

$$
A e^{j(\omega t+\phi)}-B e^{j(\omega t+\psi)}=R e^{j(\omega t+\theta)}
$$

where

$$
R=\sqrt{A^{2}+B^{2}-2 A B \cos (\phi-\psi)}
$$

and 


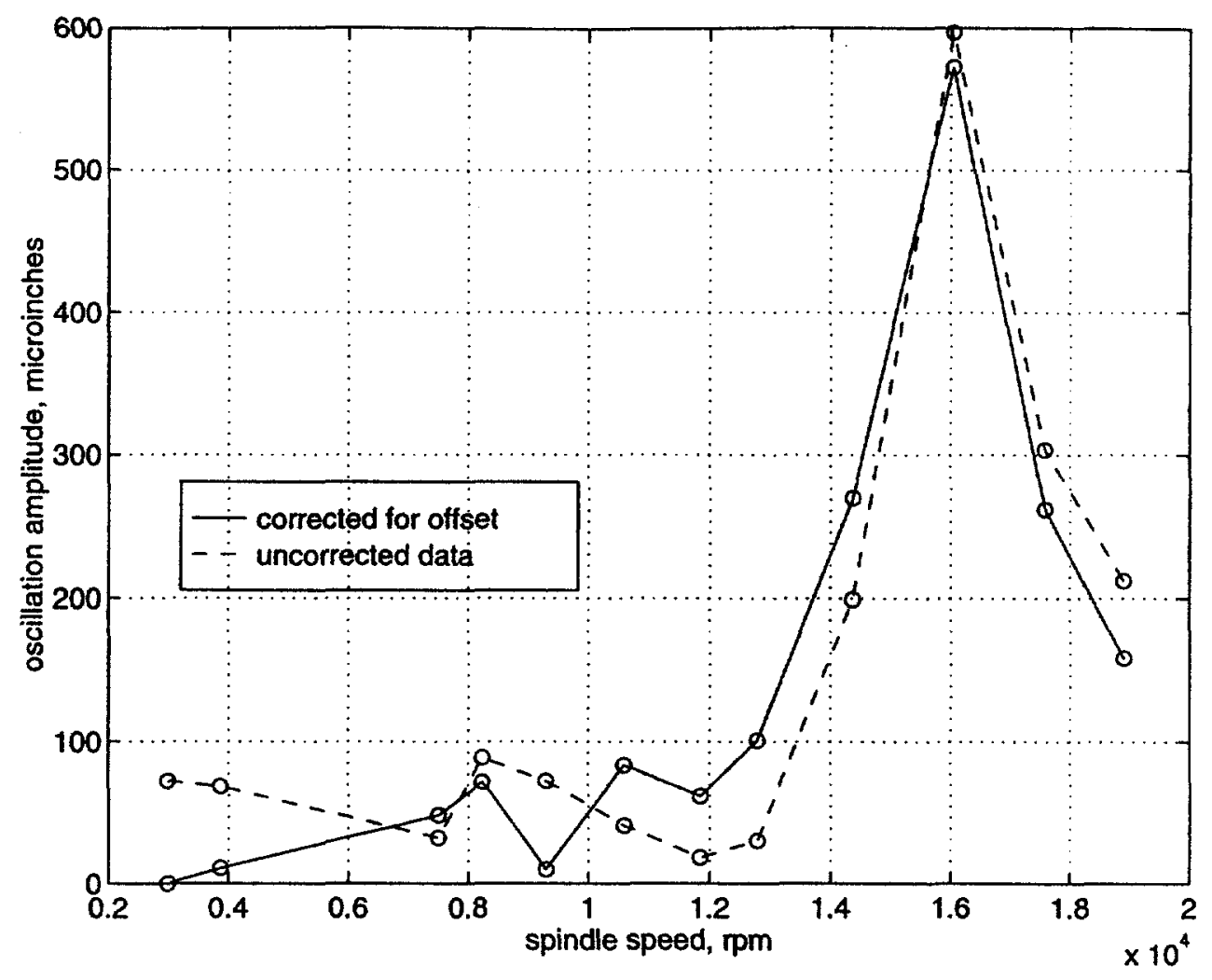

Figure C-1: Effect of correcting the capacitance gauge output for initial geometric decenter: trace of radiall ( $\mathrm{Y}$ ) cap gauge on Kistler system.

$$
\theta=\tan ^{-1}\left(\frac{A \sin \phi-B \sin \psi}{A \cos \phi-B \cos \psi}\right)
$$

An example of the result of applying this formula to the data can be seen in figure C1. This graph shows the output of the radial1 capacitance gauge for the Kistler load cell system over a speed range, for both the uncorrected and corrected data sets. At the large amplitudes (600 microinches at $16,000 \mathrm{rpm}$ ) there is not much difference between corrected and uncorrected data, but at lower speeds (i.e. $3,000 \mathrm{rpm}$ ), almost all of the signal oscillation is due to geometric decenter. Once corrected, the amplitude at these speeds drops near zero, suggesting that unbalance plays a minimal role at low speeds compared to geometric decenter. Also interesting in this particular example is the evidence of a possible harmonic oscillation at 10,600 rpm which does not appear in the uncorrected data. 


\section{Appendix D}

\section{Comparing Westwind Stiffness with Load Cell Stiffness}

To compare the apparent stiffness at the spindle shaft to the rotational stiffness of the load cell, I converted the spindle translational stiffness into an equivalent rotational stiffness about the load cell center.

For small displacements $x$ the rotational stiffness about a point can be expressed in terms of the translational stiffness by

$$
K_{r}=K d^{2}
$$

where $K_{r}$ is the effective rotational stiffness, $K$ is the translational stiffness, and $d$ is the moment arm length. This equation can be easily derived by considering a translational spring at the end of a fixed moment arm and finding an equivalent $K_{r}$ based on the spring constant $K$. Using this equation, we can compute an approximate rotational stiffness about the load cell center for the air bearing. This allows direct comparison of the air bearing stiffness to the load cell rotational stiffnesses which dominate the Westwind-JR3 modes.

The manufacturer's apparent translational stiffness at the end of the shaft at 10,000 rpm is $4.5 \times 10^{7}$ Newtons per meter. Using 0.25 meters as the moment arm distance, $d$, the effective stiffness of the spindle shaft about load cell center is approximately $3 \times 10^{6}$ Newton-meters per radian, which can be compared with about $4 \times 10^{6} \mathrm{~N} \mathrm{~m} / \mathrm{rad}$ for the load cell rotational stiffnesses about $\mathrm{X}$ and $\mathrm{Y}$. (See table 3.1 for more exact load cell stiffnesses.) The fact that these spring values are very close suggests that a better model would consist 
of a force driving the spindle mass (about $7 \mathrm{~kg}$ ), which is then connected to the larger stator mass (about $50 \mathrm{~kg}$ ) via the air bearing spring. Air bearing stiffness is an effect which should have been considered in the model presented in this thesis.

As a check on the spindle manufacturer's apparent shaft stiffness values, I used their values to analytically compute expected shaft deflections due to imbalance (no load cell present). I then compared those predictions with experimental data on spindle deflections due to imbalance with no load cell present. The empirical and estimated deflections agreed to within a factor of two across the range of speeds tested in these experiments, which suggests that the manufacturer's stiffness values are believable. At 5,800 rpm actual spindle deflection (no load cell) was 8 microinches, while at 18,000 rpm spindle deflection was 52 microinches. These deflections are noteworthy, and again point to the possibility of unmodelled spindle dynamics as a source of the discrepancies between the analytical model and the experimental data. A better analytical model would attempt to include these dynamics. 


\section{References}

[1] Kenneth L. Blaedel, Jared E. Bibler, and John S. Taylor. The effect of spindle perturbations on the quality of precision grinding. In Proceedings of the 11th annual meeting of the ASPE, pages 62-64, Monterey, CA, November 1996. American Society for Precision Engineering.

[2] Balance Dynamics Corporation. Baladyne IV: Selected case histories. Available through Douglas Cross, (313) 996-5750.

[3] Mark S. Darlow. Balancing of high-speed machinery: theory, methods, and experimental results. Mechanical Systems and Signal Processing, 1(1):105-134, 1987.

[4] Fredric F. Ehrich, editor. Handbook of Rotordynamics. McGraw-Hill, New York, 1992.

[5] International Organization for Standardization. Mechanical vibration - balance quality requirements of rigid rotors - part 1: Determination of permissible residual unbalance, 1986. ISO Standard 1940/1-1986 (E).

[6] G. Gawlak. Some problems connected with balancing of grinding wheels. ASME Journal of Engineering for Industry, 106:233-236, August 1984.

[7] American National Standards Institute. Axes of rotation: Methods for specifying and testing, 1985. Standard ANSI/ASME B89.3.4M - 1985.

[8] Hodge E. Jenkins and Thomas R. Kurfess. Adaptive process estimation for a grinding system. In International Mechanical Engineering Congress and Exposition, volume 57-1, pages 483-490, San Francisco, November 1995.

[9] Hodge E. Jenkins, Thomas R. Kurfess, and Richard C. Dorf. Design of a robust controller for a grinding system. IEEE Transactions on Control Systems Technology, 4(2):40-49, January 1996.

[10] Thomas R. Kurfess, October 1996. Private communication with the author.

[11] Thomas R. Kurfess, D.E. Whitney, and M.L. Brown. Verification of a dynamic grinding model. ASME Journal of Dynamic Systems, Measurement, and Control, 110(4):403409, December 1988.

[12] Michael Layne. Balancing systems and specific vibrations control for the grinding process. In Third International Grinding Conference, Fontana, WI, October 1988. Society of Manufacturing Engineers. 
[13] B.Y. Lee, Y.S. Tarng, and S.C. Ma. Modeling of the process damping force in chatter vibration. International Journal of Machine Tools and Manufacture, 35(7):951-962, 1995.

[14] Stephen J. Ludwick, Hodge E. Jenkins, and Thomas R. Kurfess. Determination of a dynamic grinding model. In International Mechanical Engineering Congress and Exposition, volume 55-2, pages 843-849, Chicago, November 1994.

[15] J.S. Rao. Rotor Dynamics. John Wiley \& Sons, New York, 1983.

[16] Lawrence F. Shampine and Mark W. Reichelt. The matlab ode suite. (Full text available through http://www.mathworks.com/.).

[17] Structural Dynamics, Inc., San Jose, CA. The STAR Users Guide.

[18] Michael R. Tehranchi, April 1997. Private communication with the author.

[19] H.K. Tonshoff, J. Peters, I. Inasaki, and T. Paul. Modelling and simulation of grinding processes. Annals of the CIRP, 41(2):677-688, 1992. 


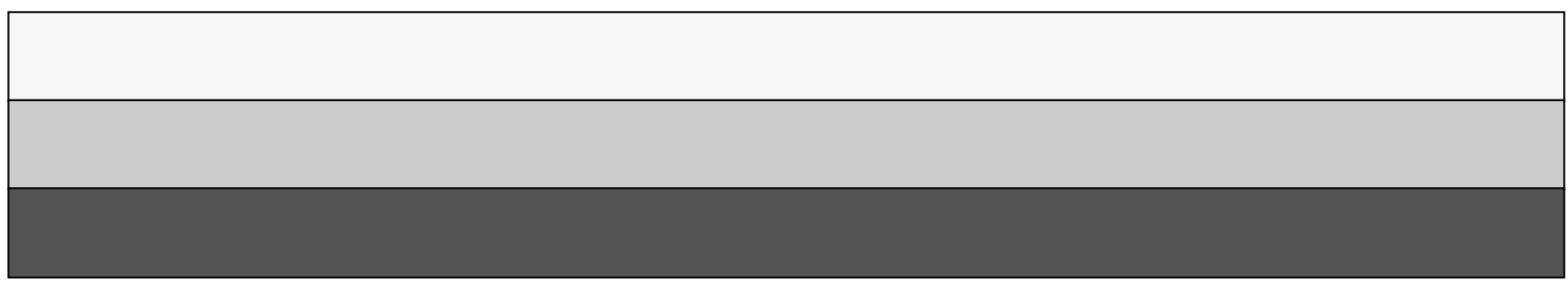

